\title{
Contribution to the knowledge on the flora of Mt. Luboten, Sharri Mts., Kosovo
}

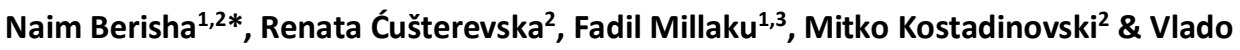 \\ Matevski ${ }^{2,4}$ \\ ${ }^{1}$ Faculty of Mathematics and Natural Sciences, University of Prishtina, Kosovo, \\ naim.berisha@uni-pr.edu \\ 2 Institute of Biology, Faculty of Natural Sciences and Mathematics, Ss. Cyril and Methodius University \\ in Skopje, North Macedonia \\ ${ }^{3}$ Faculty of Agribusiness, University "Haxhi Zeka", Peja, Kosovo \\ ${ }^{4}$ Macedonian Academy of Sciences and Arts, Skopje, North Macedonia
}

Berisha N., Ćušterevska R., Millaku F., Kostadinovski M. \& Matevski V. (2020): Contribution to the knowledge on the flora of Mt. Luboten, Sharri Mts., Kosovo. Thaiszia - J. Bot. 30 (2): 115-160.

Abstract: With the aim of improving the floristic knowledge of Kosovo, here we present an inventory of the plant taxa recorded and collected between the March 2015 and September 2019, in the mountain massif of Luboten, Sharri Mts., SE Kosovo. Field surveys were conducted repeatedly for four years, on each vegetation season. With this work we aimed to provide detailed data concerning the vascular flora richness and distributional patterns. Floristic samples were studied in all representative habitats and sites, concerning climate, exposition, altitude and bedrock composition. This research led to the identification of a total 853 plant taxa of vascular plants, belonging to 354 genera and 93 families. Among these taxa, 82 are Balkan endemics and 53 are included into the Red Book of Vascular Flora of Kosovo. Out of them, 4 are critically endangered, 16 endangered, 3 vulnerable, 8 near threatened and 22 least concern. On the basis of the available floristic literature, 2 plant taxa are found to be new for Kosovo: Bromopsis cappadocica subsp. cappadocica and Pilosella macrotricha. At a national scale, we have found new distribution records for 17 taxa and additionally for 2 plant taxa: Ranunculus fontanus and Sesleria wettsteinii, conservation suggestions are provided. All natural habitats were classified according to EUNIS and their floristic richness is herein provided. Furthermore, the corresponding EUNIS habitat(s) for each taxon of where it was recorded to be growing are also indicated.

Keywords: endemics, Kosovo, new floristic records, plant diversity, Sharri Mts. 


\section{Introduction}

In terms of scientific knowledge and conservation measures, floristic studies are of substantial importance (Calabrese et al. 2018; Gentry 1978). Conservation strategies rely heavily on significant information provided by floristic studies, particularly concerning endemic plants, their threats and distribution patterns as well, upon which priorities and action measures are set (Orsenigo et al. 2018; Martin 2009). Studies with wild vascular plants especially during the last two decades in Kosovo were intensified and they were used to evaluate the long-term changes in floristic composition (Millaku et al. 2008; Millaku et al. 2017; Prodanović et al. 2018; Rexhepi et al. 2005; Rexhepi et al. 2009; Veselaj et al. 2006; etc.), in vegetation studies on mountain ecosystems (Amiđžić et al. 2012; Berisha et al. 2019; Millaku et al. 2011; Millaku et al. 2012; Rexhepi 1994, etc.) as well as studies concerning the risk assessment ending up with the compilation of the Red list (Anonymous 2014a) and the Red Book of Vascular flora of the Republic of Kosovo (Millaku et al. 2013), among other aspects.

In the context of plant taxa richness and natural habitat diversity in Kosovo, Sharri Mts. represent a valuable and rather substantial biodiversity centre not only for Kosovo but for the whole Balkan Peninsula (Millaku et al. 2013; Micevski \& Matevski 1987; Stevanović et al. 2007; Tomović et al. 2014). This mountain chain is estimated to be home of more than 1700 vascular plant taxa (Anonymous 2014b) and 115 and 128 Balkan endemics in two corresponding UTM squares - DM87 and DM97 respectively (Tomović et al. 2014). The first massif from the north-eastern side of the Sharri Mountains is the Mountain of Luboten, which has proven to be very interesting and rich in terms of its floristic and phyto-geographic composition, based on different studies (Horvat et al. 1974; Rajevski 1990; Rexhepi 1984; Vogt et al. 2018). Mt. Luboten (in Albanian Luboten/Lybeten, in Macedonian Ljuboten) is located in the SE Kosovo at the border area between Kosovo and Northern Macedonia (Fig. 1). It represents the highest mountain massif on the NE side of the Sharri Mts. (2.498 $\mathrm{m}$ a.s.I) and as a mountain area it is characterized by unique highmountain ecosystem with a diverse regional montane to alpine flora, with plenty of endemorelic and glacial plant taxa. It differs from the surrounding mountain ranges in its bedrock composition, being mainly limestone with small and scattered silicate plates, that in other parts of the Sharri Mts. are more dominant (Anonymous 2013). With its surroundings, Luboten is also characterized by a modified continental climate with significant Mediterranean influence, reaching via the air currents through the Vardar and Lepenc valleys. Due to its high altitudes, Luboten is predominantly characterized by a typical mountain with alpine climates (Rexhepi 1984), whilst its weather also differing from other mountain massifs in the chain of Sharri Mountains.

The first floristic notes for Luboten date back on the first half of the $19^{\text {th }}$ century, when Grisebach (1843) visited the mountain, followed by Dörfler (1894-1914) and Rohlena (1937). Moreover, a large number of plant taxa that are widespread in Sharri 
Mts. are listed in the Floras of Serbia (Josifović 1970-1977; Sarić \& Diklić 1986; Diklić \& Stevanović 2012) and Northern Macedonia (Micevski 1985-2005; Matevski 2010), some of which are now being reported for Luboten. Horvat (1960) as well as Rajevski (1990) reported of some plant communities in Luboten, while Rexhepi (1984) contributed with additional floristic notes and mentions it too in the context of the general vegetation of Kosovo (Rexhepi 1994).

The research aims were to $i$. improve and update in detail the floristic knowledge of the Mt. Luboten, ii. review the presence of endemic taxa and their distribution across different habitats and iii. analyze the habitat types according to EUNIS and evaluate their general floristic richness.

In this work, the results of four year of the floristic field investigation in the Mt. Luboten and lab research are presented. According to Millaku et al. (2017) and Anonymous (2014b), the area of this mountain massif was among those in Kosovo for which the available floristic and vegetation data were particularly scarce.

\section{Material and Methods}

\section{Study area}

The study site, encompassing an area of ca $44 \mathrm{~km}^{2}$, covers the Mt. Luboten entirely, a territory of the Sharri National Park, located at the border between Kosovo and $\mathrm{N}$. Macedonia (Fig. 1). The mountain massif of Luboten is located between $42^{\circ} 11^{\prime}-$ $42^{\circ} 13^{\prime} \mathrm{N}$ and $21^{\circ} 07^{\prime}-21^{\circ} 09^{\prime} \mathrm{E}$ in Kosovo. Administratively, it belongs to the municipalities of Kaçanik and Shtërpce in Kosovo and Jegunovce in Macedonia. The altitude ranges from $310 \mathrm{~m}$ a.s.l. at the shores of Lepenci River, to $2498 \mathrm{~m}$ a.s.l. at the top of the mountain. In vertical composition, from down up scale, mixed forests between broad-leaved deciduous forest (Ostrya carpinifolia Scop., Carpinus orientalis Mill. and Fagus sylvatica L.) and coniferous forest (Abies alba Mill. and Picea abies (L.) Karst), are followed by the beech forests up to $1550 \mathrm{~m}$ a.s.l., and from there up to the summit of the mountain, it is made predominantly out of grasslands, although there are also rocky cliffs and scree. Annual rainfall ranges between 900 and $1100 \mathrm{~mm}$, and it is concentrated in the autumn-spring period with a maximum in November and a minimum in August (Çavolli 1997; Ivanović et al. 2016) and the average annual temperature of $7.6^{\circ} \mathrm{C}$ with the warmest month in summer (August) and the coldest in winter (January) (Çavolli 1997). The upper part of Mt Luboten is declared as a strict nature reserve, within the Sharri National Park (Anonymous 2015).

\section{Methods}

Field surveys were performed 32 to 36 times a year (subject to weather conditions), during four years consecutively, starting by the end of March until the end of September, with the aim of investigating the flora of Mt. Luboten. For accuracy reasons, mature plant taxa with full reproductive organs (e.g. flowers, fruits and spores) were carefully collected while immature plant material or those without 

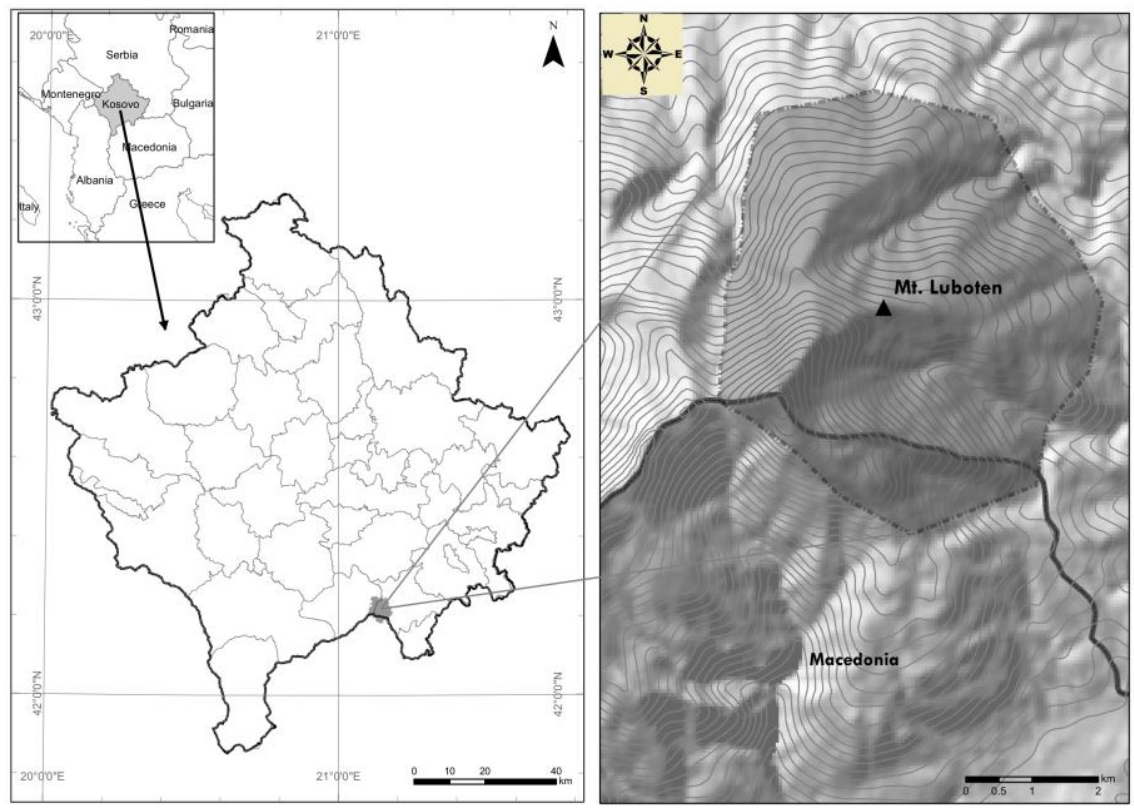

Fig. 1 Investigated area of the study.

reproductive organs at the moment of survey, were written down in a notebook, photographed and collected again in different vegetation period. Plant taxa identification and nomenclature were based mostly on Tutin \& al. (1968-1980, 1993), Flora of Serbia (Josifović 1970-1977; Sarić \& Diklić 1986; Stevanović 2012), Flora of Macedonia (Micevski 1985-2005; Matevski 2010) and Flora of Albania (Barina et al. 2018; Paparisto et al. 1988; Qosja et al. 1992, 1996; Vangjeli et al. 2000), while finally their nomenclature was updated according to Euro+Med Plantbase (Euro+Med $2006+)$. Collected plants were dried, mounted and prepared as voucher specimens following the standard guidelines (Bridson \& Forman 1998). All plant specimens are deposited in the Herbarium of the Faculty of Natural Sciences and Mathematics, University of Prishtina, given a particular reference number (Annex I). The herbarium label of each taxon provides information about the global positioning system (GPS), habitat type, collection date, local name, and scientific nomenclature. In the working table (Annex I), we have added two columns, for two particular works on Luboten: Rexhepi (1984) and Rajevski (1990), for comparison reasons of floristic data. Certain plant taxa were not mention in any of these references, but those taxa were mentioned in the "Floras of Serbia", so we did not indicate as new floristic data. In the Note column, we have listed our floristic records with the necessary data that are not mentioned anywhere on any of the currently available floristic literature sources. For identification of endemics, Tomović et al. (2014) and Petrova \& Vladimirov (2010) were followed. To verify the conservation status of each taxon, the Red Book of Vascular flora of the Republic of Kosovo (Millaku et al. 2013) and the European Red List of Vascular Plants (Bilz et al. 2011) were checked. For taxa life 
forms and chorological types, Pignatti (1982) was followed, with additional references concerning some local species to Gajić (1980) and Flora of Greece (Dimopoulos et al. 2016). Additionally, all surveyed natural habitats were classified according to EUNIS (EUNIS 2007) and each taxon is linked to its respective habitat type(s) where it was recorded. This was done mainly with the help of vegetation relevés that were done on the same time, encompasing all habitat types. The list of plant families follows that of Excursions flora of Germany (Jäger et al. 2013), while plant taxa within each family are listed alphabetically (Annex I).

\section{Results}

The checklist consists of 853 taxa (with 6 additional taxa treated separately as potentially reported in error - Tab. 1). They are distributed into 354 genera and 93 families. The most represented families are Asteraceae (98 taxa), Poaceae (69 taxa) and Caryophyllaceae (53 taxa). Among these taxa, 82 are Balkan endemics and 53 are included in the Red Book of Vascular Flora of Kosovo. Out of them, 4 were critically endangered (CR), 16 endangered (EN), 3 vulnerable (VU), 8 near threatened (NT) and 22 least concern (LC). Additionally, the European Red List of Vascular Plants, from this checklist includes 79 plant taxa, majority of them being LC (Tab. 2).

The biological spectrum of the surveyed flora shows a pronounced dominance of hemicryptophytes $(58.5 \%)$ and then geophytes $(12.3 \%)$, followed by therophytes (11.6\%), chamaephytes (8.7\%) and phanerophytes (8.7\%).

Concerning chorological types, the checklist contains Euro-Asiatic 19.4\%, Balkan $14.1 \%$, Orof. European 12.9\%, Euro-Mediterranean 9.3\%, European 8.2\%, EuroSiberian $6.6 \%$, Circumboreal $6.1 \%$, with other chorological groups being represented with less than $5 \%$.

On the basis of the available floristic literature, two plant taxa are found to be new for flora of Kosovo (Tab. 3). They are the following ones: 1. Bromopsis cappadocica (Boiss. \& Balansa) Holub subsp. cappadocica and 2. Pilosella macrotricha (Boiss.) F. W. Schultz \& Sch. Bip. Additionally, we report new distributional records for 17 plant taxa: 1. Acer heldreichii Orph. ex Boiss., 2. Arabis ciliata Clairv., 3. Arabis hirsuta (L.) Scop., 4. Arabis serpillifolia Vill., 5. Cardamine carnosa Waldst. et Kit., 6. Dianthus deltoides subsp. degenii (Bald.) Strid., 7. Dianthus gracilis subsp. armerioides (Griseb.) Tutin, 8. Erysimum comatum Pančić, 9. Festuca bosniaca Kumm. \& Sendtn., 10. Festuca koritnicensis Hayek \& J. Vetter, 11. Genista depressa M. Bieb., 12. Hieracium pilosum subsp. villosiceps Gottschl., 13. Juncus inflexus L., 14. Tanacetum larvatum (Pant.) Hayek, 15. Veronica austriaca subsp. teucrium (L.) D. A. Webb , 16. Veronica serpyllifolia L. and 17. Viola schariensis Erben.

It is worth to note that according to relevant literature sources, some of these taxa were mentioned for Sharri Mts. and two of them also for Mt. Luboten, referring to the Macedonian part of the mountain massif, as the same taxa have not been reported for the flora of Kosovo. In our survey, we have recorded them also in the Kosovo side of the massif. These taxa are: Dianthus deltoides subsp. degenii; Erysimum comatum - mentioned for Sharri Mts. in Macedonia (Micevski 1995). 
Cardamine carnosa was reported by Micevski (1995) on the macedonian side of the Mt. Luboten, Genista depressa was mentioned for Sharri Mts. in Macedonia (Micevski 2001), Juncus inflexus was mentioned for Sharri Mts. in Macedonia (Ade 1954), while Veronica serpyllifolia was mentioned for Luboten by Grisebach (1843).

Concerning the overall plant species richness and richness of the endemic plants, it turned out that Mt. Luboten is exceptionally rich in both terms. With 853 plant taxa (excluding 6 doubtful taxa), it alone accounts for more than $28 \%$ of the total of Kosovo vascular flora, since it is estimated that there are ca 3.000 of plant taxa in Kosovo (Millaku et al. 2013). Almost similar richness was observed in terms of endemic plants, given that 82 Balkan endemic taxa were recorded, as long as Kosovo is believed to have in total 367 Balkan endemic plant taxa (Tomović et al. 2014).

Regarding the EUNIS Habitat types, we have recorded 38 main habitat types, each of them divided into different sub-groups accordingly (Tab. 4). It was observed that on habitat type E1.2 (Perennial calcareous grassland and basic steppes) $29.8 \%$ of the recorded taxa were growing, followed by habitat type E4.4 (Calcareous alpine and subalpine grasslands) that held $25.9 \%$ of the recorded taxa, then E1.7 (NonMediterranean dry acid and neutral closed grassland) with 15.8\%, G1.6 (Fagus woodland) with $13.4 \%$, then E4.39 (Oro-Moesian acidophilous grasslands) with $12.5 \%$, and G1.7 (Thermophilous deciduous woodland) with $11.7 \%$. For other habitat types, associated plant taxa counted for less than $10 \%$ of the total number.

Ranunculus fontanus C. Presl was recorded in a very restricted habitat of waterlogged meadows on the mountain belt of Mt. Luboten. Population consisted of ca 40 mature individuals scattered in an area of $500 \mathrm{~m}^{2}$. This species was particularly analyzed because it is listed on the Convention for Conservation of European Wildlife and Natural Habitats (Council of Europe 1979). Furthermore, this locality represents the only species habitat in Kosovo.

Sesleria wettsteinii Dörfl. \& Hayek was recorded on a calcareous bedrock at an altitude of $2329 \mathrm{~m}$ a.s.I at a very restricted area of the alpine grasslands on the SE exposition of Luboten. It was growing in dense groups with no more than 25 mature individuals. Species area of occupancy was only $50 \mathrm{~m}^{2}$. The nearest known locality of this Balkan endemic species is in Mt. Oshlak, Sharri Mts. (Krivošej 1997) some 20 km NW from Mt. Luboten.

\section{Discussion}

Plant diversity, distribution and conservation features

The substrates' physical and chemical features are reflected on the vascular flora composition. Predominant calcareous substrates on Mt. Luboten have greatly influenced the floristic diversity, particularly noted on grasslands, a phenomenon that has also been observed in similar studies on the region: Matevski \& Kostadinovski (1999); Matevski et al. (2018) and Uzunov \& Gussev (2003). Additionally, calcifuge and acidophilous taxa are also abundant, due to the presence of silicate soils. 
Tab. 1 List of potentially doubtful plant taxa that were reported erroneously from Luboten, most probably referring to misidentified plant material.

\begin{tabular}{|c|c|c|}
\hline No. & Taxa & Comment \\
\hline 1. & $\begin{array}{l}\text { Erysimum jugicola } \\
\text { Jord. }\end{array}$ & $\begin{array}{l}\text { c. } 01 \text { - Species was reported by Rajevski (1990) but we } \\
\text { assume that it must have been reported in error, because } \\
\text { the natural distributional area of the species is France, } \\
\text { Switzerland and Italy and it is not naturally distributed into } \\
\text { the region of SE Europe. It most probably was referred to } \\
\text { misidentified plant material from: Erysimum comatum } \\
\text { Pančić. }\end{array}$ \\
\hline 2. & $\begin{array}{l}\text { Dianthus microlepis } \\
\text { Boiss. }\end{array}$ & $\begin{array}{l}\text { c. } 02 \text { - Species was reported by Rexhepi (1984) but we } \\
\text { assume that it must have been reported in error. } D \text {. } \\
\text { microlepis is to be found only on the Albanian Alps of } \\
\text { Kosovo and not on the Sharri Mts. (to whom Luboten } \\
\text { belongs to). From this group in Sharri Mts. we have only } \\
\text { Dianthus scardicus Wettst. and these two species are very } \\
\text { similar in their general appearance. Our assumption is } \\
\text { further supported by Diklić \& Stevanović (2012). }\end{array}$ \\
\hline 3. & $\begin{array}{l}\text { Dianthus petraeus } \\
\text { Waldst. \& Kit. }\end{array}$ & $\begin{array}{l}\text { c. } 03 \text { - Species was reported by Rexhepi (1984) but we } \\
\text { assume that it must have been reported in error, referring } \\
\text { to misidentified plant material. } D \text {. petraeus is distributed } \\
\text { into W and E Serbia, but it was never reported from Sharri } \\
\text { Mts. It most probably was referred to misidentified plant } \\
\text { material from: Dianthus superbus L. }\end{array}$ \\
\hline 4. & $\begin{array}{l}\text { Paronychia } \\
\text { chionaea Boiss. }\end{array}$ & $\begin{array}{l}\text { c. } 04 \text { - Species was reported by Rajevski (1990) but we } \\
\text { assume that it must have been reported in error, referring } \\
\text { to misidentified plant material of Paronychia macedonica } \\
\text { Chaudhri. Furthermore, P. chionaea is confined to N } \\
\text { Greece, S Albania and S Macedonia (?), but is absent in our } \\
\text { study region. }\end{array}$ \\
\hline 5. & $\begin{array}{l}\text { Pinguicula } \\
\text { leptoceras Rchb. }\end{array}$ & $\begin{array}{l}\text { c. } 05 \text { - Species was reported by Rajevski (1990) but we } \\
\text { assume that it must have been reported in error, referring } \\
\text { to misidentified plant material of Pinguicula balcanica } \\
\text { Casper. According to floristic studies of this genus in the } \\
\text { Balkans (Shuka et al. 2007), these two species have been } \\
\text { confused for a long time by botanists, mainly due to the } \\
\text { fact that they have very slight morphological differences, } \\
\text { for the most part on the width of their calyx lobes rounded } \\
\text { at the apex. }\end{array}$ \\
\hline 6. & $\begin{array}{l}\text { Centaurea kosaninii } \\
\text { Hayek }\end{array}$ & $\begin{array}{l}\text { c. } 06 \text { - This species was reported by Rexhepi (1984) but we } \\
\text { assume that it might have been reported in error, due to } \\
\text { the fact that C. kosaninii is an endemic serpentinophilic } \\
\text { plant species. Furthermore in Luboten there are no } \\
\text { serpentine soils, and the presence of the species on field } \\
\text { was never confirmed during our field surveys. It is however } \\
\text { present in the neighboring Mt. of Brezovica but not in } \\
\text { Luboten. }\end{array}$ \\
\hline
\end{tabular}


Tab. 2 Number of recorded plant taxa, occuring in conservation lists. XK - Kosovo, EU - Europe.

\begin{tabular}{lcc}
\hline IUCN Conservation status & XK & EU \\
\hline Extinct (EX) & & \\
Extinct in the Wild (EW) & \multicolumn{2}{c}{} \\
Critically Endangered (CR) & 4 & \\
Endangered (EN) & 16 & \\
Vulnerable (VU) & 3 & \\
Near Threatened (NT) & 8 & 3 \\
Conservation Dependent (CD) & & \\
Least Concern (LC) & 22 & 73 \\
Data Deficient (DD) & & 3 \\
Not Evaluated (NE) & & \\
\hline
\end{tabular}

\section{Discussion}

Plant diversity, distribution and conservation features

The substrates' physical and chemical features are reflected on the vascular flora composition. Predominant calcareous substrates on Mt. Luboten have greatly influenced the floristic diversity, particularly noted on grasslands, a phenomenon that has also been observed in similar studies on the region: Matevski \& Kostadinovski (1999); Matevski et al. (2018) and Uzunov \& Gussev (2003). Additionally, calcifuge and acidophilous taxa are also abundant, due to the presence of silicate soils.

Some of the recorded taxa have a very narrow distribution range: Linaria alpina (L.) Mill., Sideritis scardica Griseb. and Aubrieta columnae subsp. croatica (Schott \& al.) Mattf., are plants that inhabit dry rocky places on calcareous substrate. All these species were encountered only for Luboten and nowhere else in Kosovo. They are also enlisted as critically endangered "CR" plant taxa in the Kosovo Red Book of Vascular Flora (Millaku et al. 2013). In addition, Veronica thessalica Benth. is another critically endangered plant taxon being recorded only in Luboten and Koritnik Mts. These abovementioned plants are also Balkan Endemics, which clearly demonstrates the importance of Mt. Luboten for Kosovo's floristic diversity.

Whenever a conservation planning is undertaken, an increased emphasis is put on floristic data, plant communities and their natural habitats. Special attention is given in this context to threatened, rare and endemic plants (Kendall \& Snelson 2009). In this regard, bringing in data on the presence of two new plant taxa for the flora of Kosovo as well as new information on the distribution of seventeen other plant taxa constitutes a contribution of particular importance to the recognition of plant diversity in Kosovo.

Very interesting natural habitats, harboring plenty of endemic plants, are the vulnerable ones along the water streams at higher altitudes (E5.5 - Subalpine moist or wet tall-herb and fern stands). These habitats are proved to have very unique vegetation (Čarni \& Matevski 2010) and therefore deserve proper treatment 
Tab. 3 Data concerning the newly reported taxa for flora of Kosovo. Sampling site(s), geographical coordinates, collection dates along with other relevant data.

\begin{tabular}{|c|c|c|c|c|c|c|}
\hline No. & Plant species & Coll. dates & Herbar. & Coordinates & Sampling site(s) & Additional notes \\
\hline 1. & $\begin{array}{l}\text { Bromopsis cappadocica } \\
\text { (Boiss. \& Balansa) Holub } \\
\text { subsp. cappadocica }\end{array}$ & $\begin{array}{l}10.06 .2017 \\
28.06 .2018 \\
03.07 .2019\end{array}$ & L-072 & $\begin{array}{l}42^{\circ} 13.103 \mathrm{~N} \\
021^{\circ} 08.544 \mathrm{E}\end{array}$ & $\begin{array}{l}\text { Rocky grassland. On } \\
\text { calcareous substrate. On S, } \\
\text { SE and NE expositions alike. }\end{array}$ & $\begin{array}{l}\text { Species was growing in a range of } \\
\text { calcaeous grasslands, at altitutes } \\
\text { from } 1720 \mathrm{~m} \text {. a.s.l., up to the } \\
\text { summit of the mountain. Very well } \\
\text { developed populations. }\end{array}$ \\
\hline 2. & $\begin{array}{l}\text { Pilosella macrotricha } \\
\text { (Boiss.) F. W. Schultz \& } \\
\text { Sch. Bip. }\end{array}$ & $\begin{array}{l}21.06 .2016 \\
14.06 .2018\end{array}$ & L-724 & $\begin{array}{l}42^{\circ} 12.885 \mathrm{~N} \\
021^{\circ} 08.124 \mathrm{E}\end{array}$ & $\begin{array}{l}\text { Calcareous grasslands of the } \\
\text { S, SE \& SW slopes of the Mt. } \\
\text { Recorded on different sites, } \\
\text { from } 1812 \text { m up to } 2080 \mathrm{~m} \text {. } \\
\text { a.s.l. }\end{array}$ & $\begin{array}{l}\text { Scattered indivduals across rocky } \\
\text { gfrasslands. }\end{array}$ \\
\hline
\end{tabular}

Tab. 4 List of main recorded EUNIS Habitat types with their associated plant taxa in numbers and \%.

\begin{tabular}{lllll}
\hline No. & EUNIS & No. of taxa & $\%$ & Full EUNIS Habitat name \\
\hline 1. & D2.2 & 75 & 8.79 & Poor fens and soft-water spring mires \\
2. & D2.3 & 44 & 5.16 & Transition mires and quaking bogs \\
3. & D4.1 & 9 & 1.06 & Rich fens, eutrophic tall-herb fens \& calcareous flushes and soaks \\
4. & D4.2 & 9 & 1.06 & Basic mountain flushes and streamsides, with a rich arctic-montane flora \\
5. & D5.131 & 4 & 0.47 & Typha latifolia beds normally without free-standing water \\
6. & E1.112 & 18 & 2.11 & Sempervivum or Jovibarba communities on rock debris \\
7. & E1.2 & 254 & 29.78 & Perennial calcareous grassland and basic steppes \\
8. & E1.55 & 11 & 1.29 & Eastern sub-Mediterranean dry grassland \\
9. & E1.7 & 135 & 15.83 & Non-Mediterranean dry acid and neutral closed grassland \\
10. & E1.92 & 29 & 3.40 & Perennial open siliceous grassland \\
11. & E3.3 & 23 & 2.70 & Sub-mediterranean humid meadows \\
12. & E3.4 & 41 & 4.81 & Moist or wet eutrophic and mesotrophic grassland
\end{tabular}


Tab. 4 - cont.

\begin{tabular}{|c|c|c|c|c|}
\hline 13. & E3.5 & 22 & 2.58 & Moist or wet oligotrophic grassland \\
\hline 14. & E4.1 & 32 & 3.75 & Vegetated snow-patch \\
\hline 15. & E4.31 & 36 & 4.22 & Alpic Nardus stricta swards and related communities \\
\hline 16. & E4.34 & 50 & 5.86 & Alpigenous acidophilous grassland \\
\hline 17. & E4.39 & 107 & 12.54 & Oro-Moesian acidophilous grassland \\
\hline 18. & E4.4 & 221 & 25.91 & Calcareous alpine and subalpine grassland \\
\hline 19. & E4.5 & 21 & 2.46 & Alpine and subalpine enriched grassland \\
\hline 20. & E5.2 & 79 & 9.26 & Thermophile woodland fringes \\
\hline 21. & E5.3 & 8 & 0.94 & Pteridium aquilinum fields \\
\hline 22. & E5.4 & 77 & 9.03 & Moist or wet tall-herb and fern fringes and meadows \\
\hline 23. & E5.5 & 46 & 5.39 & Subalpine moist or wet tall-herb and fern stands \\
\hline 24. & F2.1 & 13 & 1.52 & Subarctic and alpine dwarf willow scrub \\
\hline 25. & F2.2 & 82 & 9.61 & Evergreen alpine and subalpine heath and scrub \\
\hline 26. & F2.3 & 13 & 1.52 & Subalpine deciduous scrub \\
\hline 27. & F3.2 & 69 & 8.09 & Submediterranean deciduous thickets and brushes \\
\hline 28. & G1.1 & 8 & 0.94 & Riparian and gallery woodland, with dominant Alnus, Betula, Populus or Salix \\
\hline 29. & G1.6 & 114 & 13.36 & Fagus woodland \\
\hline 30. & G1.7 & 100 & 11.72 & Thermophilous deciduous woodland \\
\hline 31. & G1.A1 & 78 & 9.14 & Quercus - Fraxinus - Carpinus betulus woodland on eutrophic and mesotrophic soils \\
\hline 32. & G3.1 & 31 & 3.63 & Abies and Picea woodland \\
\hline 33. & G4.6 & 39 & 4.57 & Mixed Abies - Picea - Fagus woodland \\
\hline 34. & $\mathrm{H} 2.3$ & 12 & 1.41 & Temperate-montane acid siliceous screes \\
\hline 35. & $\mathrm{H} 2.4$ & 41 & 4.81 & Temperate-montane calcareous and ultra-basic screes \\
\hline 36. & $\mathrm{H} 2.6$ & 32 & 3.75 & Calcareous and ultra-basic screes of warm exposures \\
\hline 37. & H3.1 & 23 & 2.70 & Acid siliceous inland cliffs \\
\hline 38. & $\mathrm{H} 3.2$ & 25 & 2.93 & Basic and ultra-basic inland cliffs \\
\hline
\end{tabular}


continuous monitoring. In some areas of the Mt. Luboten, it was noticed that water springs were altered by humans. If not managed properly, there is a great risk that these habitats will be irreversibly disrupted.

When it comes to plant conservation, it should be noted that there are two taxa that deserve additional treatment in future works, when the Kosovo Red List of Plants will be updated. Ranunculus fontanus C. Presl with a single and very narrow habitat of only 40 individuals, most probably, after conservation expert review and analysis, will have to be put in any IUCN Threatened category. Second plant species of this concern is the Balkan endemic Sesleria wettsteinii Dörfl. \& Hayek, that was recorded in an exceedingly small and confined habitat of alpine grasslands with 25 mature individuals. Taking into account its very confined habitats in Kosovo (in Sharri Mts. in Luboten and Oshlak, and in Mt. Pashtrik), a critical review of its habitats and species overall situation, with potential inclusion into the Red list, with protection measures would have been highly advisable.

\section{Life-form and Chorological spectrums}

The life-form spectrum shows a clear predominance of hemicrytophytes (58.5\%). The rather dry, calcareous substrate influences the life form spectrum of the flora, favouring hemicrytophytes. The phanerophytes are much less present (8.7\%), due to, most probably, harsh environments for the development of these species. Additionally due to less plant diversity present on the belts of mixed as well as beech forests. The chorological spectrum analysis reveals a strong prevalence of EuroAsiatic (19.4\%) influence, well balanced with local Balkan floristic element species (14.1\%), Orof. European floristic element (12.9\%) and followed by EuriMediterranean (9.3\%) and European (8.2\%) floristic elements.

\section{Conclusion}

The present work contributes to the overall knowledge of the vascular flora of the Mt. Luboten, in Sharri Mountains. Our results further confirm that this mountainous area represents a hotspot for vascular plants because of the presence of many endemic and endangered plant taxa, and furthermore, due to the presence of two newly reported taxa for flora of Kosovo. This constitution of floristic richness fully supports the areas already declared status as a strict nature reserve within the Sharri National Park. Active management projects within this area would considerably assist the better preservation of wild plant taxa. This work highlights the floristic richness of some particular habitats and points out the need to provide their enhanced protection measures.

It is necessary to promote awareness in countries public administrations regarding the potential of floristic studies, as they represent a decisive and fundamental tool that supports the sustainability and proper managing of protected areas. 
Annex I. List of recorded plant taxa on the Mt. Luboten, Sharri Mts., Kosovo.

\begin{tabular}{|c|c|c|c|c|c|c|c|c|c|c|c|}
\hline & FAMILY / Taxon & Life & Flor. element & Herb. & R.84 & R.90 & $\mathrm{XK}$ & EUNIS code & EU & END & Note \\
\hline & LYCOPODIACEAE & & & & & & & & & & \\
\hline 1. & $\begin{array}{l}\text { Huperzia selago (L.) Schrank \& Mart. } \\
\text { SELAGINELLACEAE }\end{array}$ & $\mathrm{C}$ & Subcosmop. & L-020 & $\bullet$ & $\bullet$ & EN & $\mathrm{H} 2.33$ & & & \\
\hline 2. & $\begin{array}{l}\text { Selaginella selaginoides (L.) Schrank \& Mart. } \\
\text { OPHIOGLOSSACEAE }\end{array}$ & $\mathrm{C}$ & Arctic-Alp. & L-195 & & $\bullet$ & EN & $\mathrm{D} 4.2$ & & & \\
\hline 3. & $\begin{array}{l}\text { Botrychium lunaria (L.) Sw. } \\
\text { EUQISETACEAE }\end{array}$ & G & Subcosmop. & L-001 & & $\bullet$ & & E1.7 & & & \\
\hline 4. & Equisetum arvense $\mathrm{L}$. & G & Circumbor. & L-677 & $\bullet$ & & & E3.4 & LC & & \\
\hline 5. & $\begin{array}{l}\text { Equisetum palustre } \mathrm{L} . \\
\text { DENNSTAEDIACEAE }\end{array}$ & G & Circumbor. & L-007 & $\bullet$ & & & $\mathrm{D} 2.2, \mathrm{E} 5.2$ & LC & & \\
\hline 6. & $\begin{array}{l}\text { Pteridium aquilinum (L.) Kuhn } \\
\text { PTERIDACEAE }\end{array}$ & G & Cosmopol. & L-004 & $\bullet$ & & & $\begin{array}{l}\text { G1.6C4, E5.3, E5.2, } \\
\text { G1.A1 }\end{array}$ & LC & & \\
\hline 7. & $\begin{array}{l}\text { Paragymnopteris marantae (L.) K. H. Shing } \\
\text { CYSTOPTERIDIACEAE }\end{array}$ & $\mathrm{H}$ & Subtrop. & L-002 & & & & E1.2 & NT & & \\
\hline 8. & $\begin{array}{l}\text { Cystopteris fragilis (L.) Bernh. } \\
\text { ASPLENIACEAE }\end{array}$ & $\mathrm{H}$ & Cosmopol. & L-006 & & & & $\mathrm{E} 1.2$ & & & \\
\hline 9. & Asplenium adiantum-nigrum L. & $\mathrm{H}$ & Paleotemp. & L-676 & $\bullet$ & & & F3.24311, G1.6922 & & & \\
\hline 10. & Asplenium ceterach $\mathrm{L}$. & $\mathrm{H}$ & Euro-Asiat. & L-008 & $\bullet$ & & & $\mathrm{E} 1.2, \mathrm{H} 3.2$ & LC & & \\
\hline 11. & Asplenium fissum Willd. & $\mathrm{H}$ & Orof.SE Eur. & L-009 & & & & $\begin{array}{l}\text { E4.14, E4.5, H3.216, } \\
\mathrm{H} 2.4, \mathrm{H} 2.3\end{array}$ & & & \\
\hline 12. & Asplenium ruta-muraria $\mathrm{L}$. & $\mathrm{H}$ & Circumbor. & L-010 & & & & $\mathrm{E} 1.22, \mathrm{E} 4.4, \mathrm{H} 2.4, \mathrm{~F} 3.2$ & & & \\
\hline 13. & Asplenium trichomanes $\mathrm{L}$. & $\mathrm{H}$ & Cosmopol. & L-675 & $\bullet$ & & & $\mathrm{E} 1.22, \mathrm{~F} 3.2$ & & & \\
\hline 14. & $\begin{array}{l}\text { Asplenium viride Huds. } \\
\text { ATHYRIACEAE }\end{array}$ & $\mathrm{H}$ & Circumbor. & L-011 & & & & E4.4122, E5.5 & LC & & \\
\hline 15. & Athyrium filix-femina (L.) Roth & $\mathrm{H}$ & Subcosmop. & L-012 & & & & $\begin{array}{l}\mathrm{E} 4.1, \mathrm{D} 2.3, \mathrm{D} 2.38 \\
\mathrm{D} 2.26, \mathrm{G} 3.1\end{array}$ & & & \\
\hline & BLECHNACEAE & & & & & & & & & & \\
\hline 16. & Blechnum spicant (L.) Roth & $\mathrm{H}$ & Circumbor. & L-701 & $\bullet$ & & & G1.6924 & & & \\
\hline & DRYOPTERIDIACEAE & & & & & & & & & & \\
\hline 17. & Dryopteris filix-mas (L.) Schott. & G & Subcosmop. & L-021 & $\bullet$ & & & G1.6C4, E5.2, G1.A1 & & & \\
\hline 18. & Polystichum aculeatum (L.) Roth & G & Euro-Asiat. & L-022 & $\bullet$ & & & $\mathrm{G} 1.6 \mathrm{C} 4$ & & & \\
\hline
\end{tabular}


Annex. 1 - cont

\begin{tabular}{|c|c|c|c|c|c|c|c|c|c|c|c|}
\hline & FAMILY / Taxon & Life & Flor. element & Herb. & R.84 & R.90 & $\mathrm{XK}$ & EUNIS code & EU & END & Note \\
\hline 19. & Polystichum lonchitis (L.) Roth & G & Circumbor. & L-702 & $\bullet$ & $\bullet$ & & G4.6 & & & \\
\hline 20. & $\begin{array}{l}\text { Polystichum setiferum (Forssk.) Woyn. } \\
\text { POLYPODIACEAE }\end{array}$ & G & Circumbor. & L-023 & & & & G1.A1, G4.6 & & & \\
\hline 21. & $\begin{array}{l}\text { Polypodium vulgare } \mathrm{L} . \\
\text { PINACEAE }\end{array}$ & $\mathrm{H}$ & Circumbor. & L-674 & $\bullet$ & $\bullet$ & & $F 3.2, F 3.2421$ & & & \\
\hline 22. & Abies alba Mill. & $\mathrm{P}$ & Orof.S.Eur. & L-030 & - & & & G1.6C4, G1.7C1, E5.2 & & & \\
\hline 23. & Picea abies (L.) Karst & $\mathrm{P}$ & Euro-Siber. & L-031 & & & & G1.6C4, G3.162 & & & \\
\hline 24. & $\begin{array}{l}\text { Pinus peuce Griseb. } \\
\text { CUPRESSACEAE }\end{array}$ & $\mathrm{P}$ & Balkan & L-032 & & - & & H2.6B, E3.52, G3.1 & & $\operatorname{End}(B)$ & \\
\hline 25. & Juniperus communis L. subsp. communis & $\mathrm{P}$ & Circumbor. & L-034 & - & & & F3.16, E4.34 & & & \\
\hline 26. & $\begin{array}{l}\text { Juniperus communis subsp. nana Syne. } \\
\text { ARISTOLOCHIACEAE }\end{array}$ & $\mathrm{P}$ & Euro-Asiat. & L-035 & $\bullet$ & - & & $\begin{array}{l}\text { F2.231, E4.4, E4.34, } \\
\text { H2.4 }\end{array}$ & & & \\
\hline 27. & Aristolochia pallida Willd. & G & Euri-Medit. & L-678 & - & & & G1.6922, G1.7 & & & \\
\hline 28. & $\begin{array}{l}\text { Asarum europaeum L. } \\
\text { ARACEAE }\end{array}$ & $\mathrm{H}$ & Euro-Siber. & L-195 & $\bullet$ & - & & G1.7, G3.1, G1.A1 & & & \\
\hline 29. & Arum italicum Mill. & G & Steno-Medit. & N/A & - & & & E1.22, F2.1, F2.231 & & & \\
\hline 30. & $\begin{array}{l}\text { Arum maculatum } \mathrm{L} . \\
\text { NARTHECIACEAE }\end{array}$ & G & Centro-Eur. & L-673 & $\bullet$ & & & F2.231 & & & \\
\hline 31. & $\begin{array}{l}\text { Narthecium scardicum Košanin } \\
\text { MELANTHIACEAE }\end{array}$ & G & Balkan & L-005 & & & NT & $\begin{array}{l}\text { D2.28, D2.38, D2.26, } \\
\text { D4.2 }\end{array}$ & & $\operatorname{End}(B)$ & \\
\hline 32. & Veratrum lobelianum Bernh. & G & Euro-Siber. & L-025 & & & & E4.4, E4.412 & & & \\
\hline 33. & $\begin{array}{l}\text { Paris quadrifolia } \mathrm{L} \text {. } \\
\text { COLCHICACEAE }\end{array}$ & G & Euro-Asiat. & L-679 & - & & & $\mathrm{G} 4.6$ & & & \\
\hline 34. & $\begin{array}{l}\text { Colchicum autumnale L. } \\
\text { DIOSCORIACEAE }\end{array}$ & G & Centro-Eur. & L-003 & - & & & E1.2, E1.22 & & & \\
\hline 35. & $\begin{array}{l}\text { Dioscorea communis (L.) Caddick \& Wilkin } \\
\text { LILIACEAE }\end{array}$ & G & Euri-Medit. & L-672 & - & & & $\mathrm{F} 2.11, \mathrm{G} 1.6 \mathrm{C} 4$ & & & \\
\hline 36. & Erythronium dens-canis L. & G & Orof.S.Eur. & L-013 & - & & & $\begin{array}{l}\text { G1.6922, G1.7C1, E5.5, } \\
\text { E5.2 }\end{array}$ & & & \\
\hline 37. & Gagea lutea (L.) Ker Gawl. & G & Euro-Siber. & L-014 & & & & F2, G4.6 & & & \\
\hline 38. & Lilium albanicum Griseb. & G & Balkan & L-015 & - & - & LC & F2.263, E4.116, E4.5 & & End(B) & \\
\hline 39. & $\begin{array}{l}\text { Lilium martagon L. } \\
\text { ORCHIDACEAE }\end{array}$ & G & Euro-Asiat. & L-016 & $\bullet$ & & & $\begin{array}{l}\text { F2.263, F2.3, F2.22, } \\
\text { H2.3, G1.A1 }\end{array}$ & & & \\
\hline
\end{tabular}


Annex. 1 - cont.

\begin{tabular}{|c|c|c|c|c|c|c|c|c|c|c|}
\hline & FAMILY / Taxon & Life & Flor. element & Herb. & R.84 & R.90 & $\mathrm{XK}$ & EUNIS code & EU & END \\
\hline 40. & $\begin{array}{l}\text { Anacamptis laxiflora (Lam.) R. M. Bateman, Pridgeon \& M. } \\
\text { W. Chase }\end{array}$ & G & Euri-Medit. & $\mathrm{N} / \mathrm{A}$ & $\bullet$ & & & E5.5723 & LC & \\
\hline 41. & $\begin{array}{l}\text { Anacamptis morio (L.) R. M. Bateman, Pridgeon \& M. W. } \\
\text { Chase }\end{array}$ & G & Euro-Cauc. & L-680 & $\bullet$ & & & E5.5723, E4.41731 & NT & \\
\hline 42. & $\begin{array}{l}\text { Anacamptis palustris (Jacq.) R. M. Bateman, Pridgeon \& M. } \\
\text { W. Chase }\end{array}$ & G & Euro-Asiat. & N/A & $\bullet$ & & & $\mathrm{D} 2.282$ & & \\
\hline 43. & Anacamptis pyramidalis (L.) Rich. & G & Euri-Medit. & L-671 & $\bullet$ & & & $\mathrm{E} 4.4, \mathrm{E} 4.4121$ & LC & \\
\hline 44. & Dactylorhiza cordigera (Fr.) Soó & G & Balkan & L-041 & & & & $\mathrm{D} 2.2, \mathrm{D} 2.282$ & LC & End(B) \\
\hline 45. & Dactylorhiza maculata (L.) Soó & G & Paleotemp. & L-042 & & & & $\mathrm{G} 1.6 \mathrm{C} 4, \mathrm{G} 4.6, \mathrm{D} 2.2$ & LC & \\
\hline 46. & Dactylorhiza sambucina (L.) Soó & G & Euro-Cauc. & L-043 & & & & $\mathrm{D} 2.2, \mathrm{D} 2.282, \mathrm{G} 4.6$ & LC & \\
\hline 47. & $\begin{array}{l}\text { Dactylorhiza viridis (L.) R. M. Bateman, Pridgeon \& M. W. } \\
\text { Chase }\end{array}$ & G & Circumbor. & N/A & & $\bullet$ & & G1.7, G1.6C4 & LC & \\
\hline 48. & Gymnadenia conopsea (L.) R. Br. & G & Euro-Asiat. & L-681 & $\bullet$ & & & $\mathrm{E} 4, \mathrm{E} 4.31$ & LC & \\
\hline 49. & Gymnadenia friwaldii Rchb. & G & Balkan & L-044 & & & NT & $\begin{array}{l}\text { E3.4, D2.3, D2.38, } \\
\text { D2.26 }\end{array}$ & LC & \\
\hline 50. & Gymnadenia nigra (L.) Rchb.f. & G & Euri-Medit. & L-045 & $\bullet$ & $\bullet$ & & $\mathrm{E} 3.52, \mathrm{E} 4$ & LC & \\
\hline 51. & Limodorum abortivum (L.) Sw. & G & Medit. & N/A & $\bullet$ & & & F2.231, G1.6933 & LC & \\
\hline 52. & Neotinea maculata (Desf.) Stearn & G & Medit. & L-046 & & & & $\begin{array}{l}\mathrm{F} 2.263, \mathrm{~F} 2.231, \mathrm{D} 2.38 \\
\mathrm{D} 2.26\end{array}$ & LC & \\
\hline 53. & $\begin{array}{l}\text { Neotinea tridentata (Scop.) R. M. Bateman, Pridgeon \& M. } \\
\text { W. Chase }\end{array}$ & G & Medit. & N/A & $\bullet$ & & & E4 & LC & \\
\hline 54. & Orchis purpurea Huds. & G & Euro-Asiat. & L-682 & $\bullet$ & & & E3.4, E4.4, F2.22 & LC & \\
\hline 55. & $\begin{array}{l}\text { Platanthera bifolia (L.) Rich. } \\
\text { IRIDACEAE }\end{array}$ & G & Paleotemp. & L-683 & $\bullet$ & & & E3.52, E4, F2.231 & LC & \\
\hline 56. & Crocus biflorus subsp. weldenii (Hoppe \& Fürnr.) K. Richt. & G & Medit. & L-196 & $\bullet$ & & & $\mathrm{E} 1.22, \mathrm{~F} 2.23$ & & \\
\hline 57. & Crocus kosaninii Pulevic & G & Submedit. & N/A & $\bullet$ & & EN & G1.7 & & End(B) \\
\hline 58. & Crocus veluchensis Herb. & G & Balkan & L-036 & $\bullet$ & $\bullet$ & & $\mathrm{E} 4.3, \mathrm{E} 4.3411$ & & End(B) \\
\hline 59. & Crocus vernus (L.) Hill. subsp. vernus & G & Medit. & L-037 & & & & G1.6933, E4.3 & & \\
\hline 60. & Iris graminea $\mathrm{L}$. & G & Euri-Medit. & L-178 & $\bullet$ & & & G1.6933 & & \\
\hline 61. & Iris pseudacorus L. & G & Euro-Asiat. & N/A & $\bullet$ & & & D2.2 & LC & \\
\hline 62. & $\begin{array}{l}\text { Iris reichenbachii Heuff. } \\
\text { AMARYLLIDACEAE }\end{array}$ & G & Balkan & L-179 & $\bullet$ & & & G4.6 & & \\
\hline 63. & Allium carinatum L. subsp. carinatum & G & Europ. & L-684 & & $\bullet$ & & $\mathrm{E} 4.3, \mathrm{E} 4.39$ & LC & \\
\hline 64. & $\begin{array}{l}\text { Allium carinatum subsp. pulchellum (G. Don) Bonnier \& } \\
\text { Layens }\end{array}$ & G & Europ. & $\mathrm{L}-017$ & & $\bullet$ & & $\mathrm{E} 4.3, \mathrm{E} 4.39$ & & \\
\hline 65. & Allium ursinum $\mathrm{L}$. & G & Euro-Asiat. & L-018 & & & & G1.6933, G3.1 & LC & \\
\hline 66. & Allium victorialis $\mathrm{L}$. & G & Circumbor. & N/A & $\bullet$ & & & $\mathrm{F} 2.231$ & LC & \\
\hline
\end{tabular}


Annex. 1 - cont.

\begin{tabular}{|c|c|c|c|c|c|c|c|c|c|c|c|}
\hline & FAMILY / Taxon & Life & Flor. element & Herb. & R.84 & R.90 & XK & EUNIS code & EU & END & Note \\
\hline 67. & Allium vineale $\mathrm{L}$. & G & Euri-Medit. & $\mathrm{N} / \mathrm{A}$ & $\bullet$ & & & E5.2 & LC & & \\
\hline \multirow[t]{2}{*}{68.} & Galanthus nivalis L. & G & Euro-Cauc. & L-019 & $\bullet$ & & & G1.6933 & NT & & \\
\hline & ASPARAGACEAE & & & & & & & & & & \\
\hline 69. & Muscari botryoides (L.) Mill. & G & Euri-Medit. & $\mathrm{L}-470$ & $\bullet$ & & & G1.69322, E4 & & & \\
\hline 70. & Muscari racemosum Mill. & G & Submedit. & L-180 & $\bullet$ & & & $\mathrm{E} 4, \mathrm{E} 4.116$ & & & \\
\hline 71. & Asparagus tenuifolius Lam. & C & Euri-Medit. & N/A & $\bullet$ & & & G1.7C1, G4.6 & $\mathrm{LC}$ & & \\
\hline 72. & Convallaria majalis L. & G & Euro-Siber. & L-038 & $\bullet$ & & & G4.6, G1.6933 & & & \\
\hline 73. & Ornithogalum comosum L. & G & Euro-Asiat. & N/A & $\bullet$ & & & E1.55 & & & \\
\hline 74. & $\begin{array}{l}\text { Ornithogalum orthophyllum subsp. kochii (Parl.) Maire \& } \\
\text { Weiller }\end{array}$ & G & Steno-Medit. & L-039 & $\bullet$ & & & E4, E4.3411 & & & \\
\hline 75. & Ornithogalum umbellatum L. & G & Steno-Medit. & L-471 & $\bullet$ & & & E4.3, E4.39 & & & \\
\hline 76. & Polygonatum odoratum (Mill.) Druce & G & Euro-Siber. & L-686 & $\bullet$ & & & G1.6933 & & & \\
\hline 77. & Polygonatum verticillatum (L.) All. & G & Euro-Asiat. & $\mathrm{N} / \mathrm{A}$ & $\bullet$ & & & G1.6933 & & & \\
\hline 78. & Prospero autumnale (L.) Speta & G & Steno-Medit. & N/A & $\bullet$ & & & E4.4 & & & \\
\hline \multirow[t]{2}{*}{79.} & Scilla bifolia $\mathrm{L}$. & G & Euro-Cauc. & L-040 & $\bullet$ & & & G1.6933 & & & \\
\hline & TYPHACEAE & & & & & & & & & & \\
\hline \multirow[t]{2}{*}{80.} & Typha latifolia L. & G & Cosmopol. & L-472 & $\bullet$ & & & D5.131 & LC & & \\
\hline & JUNCACEAE & & & & & & & & & & \\
\hline 81. & Juncus articulatus L. & G & Circumbor. & L-670 & $\bullet$ & & & $\mathrm{D} 2.2, \mathrm{E} 3.3$ & LC & & \\
\hline 82. & Juncus conglomeratus L. & $\mathrm{H}$ & Europ. & L-047 & $\bullet$ & & & $\begin{array}{l}\text { E3.52, E5.5721, D2.38, } \\
\text { D2.26 }\end{array}$ & & & \\
\hline 83. & Juncus effusus L. & $\mathrm{H}$ & Europ. & L-048 & & & & $\mathrm{D} 2.2, \mathrm{E} 5.2$ & LC & & \\
\hline 84. & Juncus inflexus L. & $\mathrm{H}$ & Paleotemp. & $\mathrm{L}-714$ & & & & E3.4, E3.5, D2.2 & & & $\nabla$ \\
\hline 85. & Juncus thomasii Ten. & G & Europ. & L-049 & & & & $\begin{array}{l}\text { D2.2, E5.5, E5.5721, } \\
\text { D2.38, D2.26 }\end{array}$ & & & \\
\hline 86. & Juncus trifidus L. & G & Arctic-Alp. & L-473 & & $\bullet$ & & E4.31, E4.14, E4.5 & & & \\
\hline 87. & Luzula campestris (L.) DC. & $\mathrm{H}$ & Euro-Cauc. & L-050 & $\bullet$ & $\bullet$ & & $\mathrm{E} 1.2, \mathrm{E} 1.7, \mathrm{E} 4.34$ & & & \\
\hline 88. & Luzula forsteri (Sm.) DC. & $\mathrm{H}$ & Euri-Medit. & L-051 & $\bullet$ & & & G1.6C4, G3.1 & & & \\
\hline 89. & Luzula luzuloides (Lam.) Dandy \& Wilmott & $\mathrm{H}$ & Europ. & L-715 & & & & $\mathrm{E} 4.3, \mathrm{E} 4.39, \mathrm{E} 4.312$ & & & \\
\hline 90. & Luzula multiflora (Ehrh.) Lej. subsp. multiflora & $\mathrm{H}$ & Circumbor. & N/A & & $\bullet$ & & E1.2, E1.7 & & & \\
\hline 91. & Luzula exspectata Bacic \& Jogan & $\mathrm{H}$ & Balkan & L-053 & & & & $\mathrm{E} 5.5, \mathrm{E} 4.413 \mathrm{~A}$ & & & \\
\hline 92. & Luzula spicata (L.) D.C. & $\mathrm{H}$ & Orof.SE Eur. & L-052 & $\bullet$ & $\bullet$ & & $\mathrm{E} 1.7, \mathrm{E} 4.3$ & & & \\
\hline \multirow[t]{2}{*}{93.} & Luzula sylvatica (Huds) Gaudin. & $\mathrm{H}$ & Orof.SE Eur. & L-054 & $\bullet$ & $\bullet$ & & $\mathrm{F} 2.2, \mathrm{E} 4.41, \mathrm{E} 4.34$ & & & \\
\hline & CYPERACEAE & & & & & & & & & & \\
\hline 94. & Blysmus compressus (L.) Link & G & Euro-Siber. & L-055 & & & & $\begin{array}{l}\text { D2.282, D4.1, D2.38, } \\
\text { D2.26, D4.2 }\end{array}$ & & & \\
\hline 95. & Carex atrata L. subsp. atrata & $\mathrm{H}$ & Arctic-Alp. & L-474 & $\bullet$ & & & E4.392 & & & \\
\hline
\end{tabular}


Annex. 1 - cont.

\begin{tabular}{|c|c|c|c|c|c|c|c|c|c|c|c|}
\hline & FAMILY / Taxon & Life & Flor. element & Herb. & R.84 & R.90 & XK & EUNIS code & EU & END & Note \\
\hline 96. & Carex atrata subsp. aterrima (Hoppe) Čelak. & $\mathrm{H}$ & Euro-Asiat. & L-056 & $\bullet$ & & & E4.392, E4.4121 & & & \\
\hline 97. & Carex bulgarica (Domin) Lazare & $\mathrm{H}$ & Balkan & L-067 & & $\bullet$ & & E4.3, E4.39, E4.34 & & & \\
\hline 98. & Carex caryophyllea Latourr. & $\mathrm{H}$ & Euro-Siber. & L-057 & & $\bullet$ & & $\mathrm{E} 1.2, \mathrm{E} 1.22, \mathrm{E} 4.312$ & & & \\
\hline 99. & Carex curvula All. & $\mathrm{H}$ & Orof.S.Eur. & L-058 & $\bullet$ & & & E4.3, E4.3411 & & & \\
\hline 100. & Carex distans L. & $\mathrm{H}$ & Europ. & N/A & $\bullet$ & & & E3.4, E3.3 & & & \\
\hline 101. & Carex echinata Murray & $\mathrm{H}$ & Circumtemp. & L-060 & $\bullet$ & & & $\begin{array}{l}\text { D2.22, E5.2, D2.38, } \\
\text { D2.26 }\end{array}$ & & & \\
\hline 102. & Carex ferruginea Scop. & G & Orof.S.Eur. & N/A & $\bullet$ & & & E4.392 & & & \\
\hline 103. & Carex flava $\mathrm{L}$. & $\mathrm{H}$ & Circumtemp. & L-061 & $\bullet$ & & & $\begin{array}{l}\text { D2.2, D2.22, D2.3, } \\
\text { D2.38, D2.26 }\end{array}$ & & & \\
\hline 104. & Carex hirta L. & G & Euro-Asiat. & N/A & $\bullet$ & & & E3.4 & & & \\
\hline 105. & Carex kitaibeliana Degen ex Bechere. & $\mathrm{H}$ & Orof.SE.Eur. & L-063 & $\bullet$ & $\bullet$ & & $\mathrm{E} 4.4, \mathrm{~F} 2.1, \mathrm{H} 2.4$ & & & \\
\hline 106. & Carex leporina L. & $\mathrm{H}$ & Euro-Siber. & L-064 & $\bullet$ & & & $\begin{array}{l}\text { E3.5, D2.2, D2.3, D2.38, } \\
\text { D2.26 }\end{array}$ & & & \\
\hline 107. & Carex nigra (L.) Reichard & G & Subcosmop. & L-065 & & & & $\begin{array}{l}\text { D2.22, D2.282, D2.38, } \\
\text { D4.2 }\end{array}$ & & & \\
\hline 108. & Carex ornithopoda Willd. & $\mathrm{H}$ & Euro-Cauc. & N/A & & $\bullet$ & & E4.392 & & & \\
\hline 109. & Carex pilulifera $\mathrm{L}$. & $\mathrm{H}$ & Europ. & N/A & $\bullet$ & & & E1.2 & & & \\
\hline 110. & Carex pyrenaica Wahlenb. & $\mathrm{H}$ & Euro-Asiat. & N/A & $\bullet$ & & & E1.2 & & & \\
\hline 111. & Carex rupestris All. & $\mathrm{H}$ & Circumbor. & L-066 & & $\bullet$ & & $\mathrm{E} 4.4, \mathrm{E} 4.41$ & & & \\
\hline 112. & Carex sylvatica Huds. & $\mathrm{H}$ & Euro-Asiat. & L-068 & & & & $\begin{array}{l}\text { G1.6C4, G1.7C1, E5.2, } \\
\text { G1.A1 }\end{array}$ & & & \\
\hline 113. & Eriophorum angustifolium Honck. & G & Circumbor. & L-069 & & & & $\begin{array}{l}\text { D2.22, D2.26, D2.282, } \\
\text { D2.38 }\end{array}$ & LC & & \\
\hline 114. & $\begin{array}{l}\text { Eriophorum latifolium Hoppe. } \\
\text { POACEAE }\end{array}$ & $\mathrm{H}$ & Euro-Asiat. & L-070 & & & & $\mathrm{D} 2.2, \mathrm{D} 2.3, \mathrm{D} 2.38$ & & & \\
\hline 115. & Aegilops triuncialis L. & $\mathrm{T}$ & Euro-Asiat. & N/A & $\bullet$ & & & E1.7 & LC & & \\
\hline 116. & Agrostis canina L. & $\mathrm{H}$ & Euro-Siber. & L-026 & $\bullet$ & & & $\mathrm{D} 2.2, \mathrm{D} 2.22, \mathrm{E} 5.2$ & LC & & \\
\hline 117. & Agrostis capillaris L. & $\mathrm{H}$ & Euro-Siber. & N/A & $\bullet$ & $\bullet$ & & E1.2, E1.22, E3.3 & & & \\
\hline 118. & Agrostis stolonifera $\mathrm{L}$. & $\mathrm{H}$ & Circumbor. & L-027 & & & & E1.55, E5.2, G1.1 & LC & & \\
\hline 119. & Alopecurus pratensis $\mathrm{L}$. & $\mathrm{H}$ & Euro-Siber. & L-475 & $\bullet$ & & & $\mathrm{D} 2.2, \mathrm{E} 3.3$ & LC & & \\
\hline 120. & Alopecurus rendlei Eig & $\mathrm{T}$ & Euri-Medit. & N/A & $\bullet$ & & & E3.4 & & & \\
\hline 121. & Anthoxanthum odoratum L. & $\mathrm{H}$ & Euro-Asiat. & L-028 & $\bullet$ & $\bullet$ & & $\begin{array}{l}\text { E4.41, E4.116, E5.2, } \\
\text { E4.34, E3.3 }\end{array}$ & & & \\
\hline 122. & Avenella flexuosa (L.) Drejer & $\mathrm{H}$ & Subcosmop. & L-029 & $\bullet$ & $\bullet$ & & E1.73, E4.34, G3.1 & & & \\
\hline 123. & Avenula pubescens (Huds.) Dumort. & $\mathrm{H}$ & Euro-Asiat. & N/A & & $\bullet$ & & E4.1, E4.5 & & & \\
\hline 124. & Bellardiochloa variegata (Lam.) Kerguélen & $\mathrm{H}$ & Europ. & L-071 & $\bullet$ & $\bullet$ & & E4, E4.14, E4.34 & & & \\
\hline
\end{tabular}


Annex. 1 - cont.

\begin{tabular}{|c|c|c|c|c|c|c|c|c|c|c|c|}
\hline & FAMILY / Taxon & Life & Flor. element & Herb. & R.84 & R.90 & $\mathrm{XK}$ & EUNIS code & EU & END & Note \\
\hline 125. & Brachypodium sylvaticum (Huds.) P. Beauv. & $\mathrm{H}$ & Paleotemp. & L-669 & $\bullet$ & & & $\mathrm{D} 2.26, \mathrm{E} 1.2, \mathrm{G} 1 . \mathrm{A} 1$ & & & \\
\hline 126. & Briza media L. & $\mathrm{H}$ & Euro-Siber. & L-223 & $\bullet$ & & & $\mathrm{E} 1.2, \mathrm{E} 1.73$ & & & \\
\hline 127. & $\begin{array}{l}\text { Bromopsis cappadocica (Boiss. \& Balansa) Holub subsp. } \\
\text { cappadocica }\end{array}$ & $\mathrm{H}$ & Euro-Asiat. & L-072 & & & & $\mathrm{E} 1.2, \mathrm{E} 1.22$ & & & $\square$ \\
\hline 128. & Bromopsis erecta (Huds.) Fourr. & $\mathrm{H}$ & Paleotemp. & L-073 & $\bullet$ & $\bullet$ & & $\mathrm{E} 1.22, \mathrm{E} 4.4, \mathrm{E} 4.41, \mathrm{H} 2.4$ & & & \\
\hline 129. & Bromopsis riparia (Rehmann) Holub & $\mathrm{H}$ & Euro-Asiat. & N/A & $\bullet$ & & & E1.2 & & & \\
\hline 130. & Bromus racemosus $\mathrm{L}$. & $\mathrm{T}$ & Europ. & L-074 & $\bullet$ & & & $\mathrm{E} 1.2, \mathrm{E} 5.2, \mathrm{E} 3.3$ & & & \\
\hline 131. & Calamagrostis arundinacea (L.) Roth & $\mathrm{H}$ & Euro-Asiat. & L-075 & $\bullet$ & $\bullet$ & & F2.2, E4.3, E4.39, E5.2 & & & \\
\hline 132. & Calamagrostis epigejos (L.) Roth & $\mathrm{H}$ & Euro-Siber. & L-076 & & & & $\mathrm{E} 1.7, \mathrm{E} 1.74$ & & & \\
\hline 133. & Calamagrostis varia (Schrad.) Host subsp. varia & $\mathrm{H}$ & Europ. & L-077 & & & & E5.5, E5.51 & & & \\
\hline 134. & Cynodon dactylon (L.) Pers. & G & Cosmopol. & L-476 & $\bullet$ & & & G1.6C4 & & & \\
\hline 135. & Cynosurus cristatus L. & $\mathrm{H}$ & Euro-Asiat. & N/A & $\bullet$ & & & $\mathrm{E} 1.2, \mathrm{E} 5.3$ & & & \\
\hline 136. & Cynosurus echinatus L. & $\mathrm{T}$ & Euri-Medit. & N/A & $\bullet$ & & & E5.3 & & & \\
\hline 137. & Dactylis glomerata $\mathrm{L}$. & $\mathrm{H}$ & Paleotemp. & L-477 & $\bullet$ & & & E1.2, E3.4, G1.A1 & & & \\
\hline 138. & Danthonia alpina Vest & $\mathrm{H}$ & Europ. & L-668 & $\bullet$ & & & $\mathrm{E} 1.2, \mathrm{~F} 2.231$ & & & \\
\hline 139. & Danthonia decumbens (L.) DC. & $\mathrm{H}$ & Europ. & L-685 & & & & $\mathrm{F} 2.1, \mathrm{E} 4.1, \mathrm{E} 4.5$ & & & \\
\hline 140. & Deschampsia cespitosa (L.) P. Beauv. & $\mathrm{H}$ & Cosmopol. & L-078 & $\bullet$ & & & $\begin{array}{l}\text { D2.2, D2.22, E3.4, } \\
\text { D2.38 }\end{array}$ & & & \\
\hline 141. & Festuca adamovicii (St. Yves.) Markgr.-Dann. & $\mathrm{H}$ & Balkan & L-079 & & $\bullet$ & & $\begin{array}{l}\text { E4.41, E4.39, E4.4126, } \\
\text { E1.112 }\end{array}$ & & End(B) & \\
\hline 142. & Festuca bosniaca Kumm. \& Sendtn. & $\mathrm{H}$ & Medit. & L-080 & & & & E4.41 & & & $\nabla$ \\
\hline 143. & Festuca halleri subsp. scardica (Griseb.) Markgr.-Dann. & $\mathrm{H}$ & Orof.S.Eur. & L-478 & & $\bullet$ & & E4.3, E4.31, E4.39, H2.4 & & End(B) & \\
\hline 144. & Festuca heterophylla Lam. & $\mathrm{H}$ & Euro-Cauc. & N/A & $\bullet$ & & & $\mathrm{E} 1.7, \mathrm{~F} 2.231, \mathrm{G} 1 . \mathrm{A} 1$ & LC & & \\
\hline 145. & Festuca korabensis (Markgr.-Dann.) Markgr.-Dann. & $\mathrm{H}$ & Orof.SE.Eur. & N/A & $\bullet$ & $\bullet$ & & E1.2 & & & \\
\hline 146. & Festuca koritnicensis Hayek \& J. Vetter & $\mathrm{H}$ & Balkan & L-081 & & & & E4.4, E4.41 & & End(B) & $\nabla$ \\
\hline 147. & Festuca panciciana (Hack.) K. Richt. & $\mathrm{H}$ & Balkan & L-082 & $\bullet$ & & & $\begin{array}{l}\text { E1.2, E1.22, E4.4, E4.34, } \\
\text { H2.4 }\end{array}$ & & & \\
\hline 148. & Festuca rubra L. subsp. rubra & $\mathrm{H}$ & Circumbor. & N/A & & $\bullet$ & & E1.2, E1.7, E3.5 & & & \\
\hline 149. & Festuca violacea Gaudin & $\mathrm{H}$ & Orof.SE.Eur. & L-479 & $\bullet$ & $\bullet$ & & E4.3, E4.39, E4.392 & & & \\
\hline 150. & Helictochloa versicolor (Vill.) Romero Zarco & $\mathrm{H}$ & Orof.S.Eur. & N/A & $\bullet$ & $\bullet$ & & E4 & & & \\
\hline 151. & Holcus lanatus L. & $\mathrm{H}$ & Circumbor. & L-480 & $\bullet$ & & & E3.4 & & & \\
\hline 152. & Hordeum bulbosum L. & $\mathrm{H}$ & Subtrop. & L-481 & $\bullet$ & & & E5.2 & LC & & \\
\hline 153. & Hordeum secalinum Schreb. & $\mathrm{H}$ & Euri-Medit. & N/A & $\bullet$ & & & E3.3 & LC & & \\
\hline 154. & Koeleria eriostachya Panc. & $\mathrm{H}$ & Orof.SE.Eur. & L-083 & $\bullet$ & $\bullet$ & & $\mathrm{E} 1.2, \mathrm{E} 4.4, \mathrm{E} 4.41$ & & & \\
\hline 155. & Koeleria lobata (M. Bieb.) Roem. \& Schult. & $\mathrm{H}$ & Medit.-Mont. & L-084 & & & & $\begin{array}{l}\text { E1.2, E1.22, E1.55, } \\
\text { E4.34 }\end{array}$ & & & \\
\hline 156. & Lolium perenne L. & $\mathrm{H}$ & Circumbor. & L-482 & $\bullet$ & & & E1.2, E1.55, E3.3 & LC & & \\
\hline
\end{tabular}


Annex. 1 - cont.

\begin{tabular}{|c|c|c|c|c|c|c|c|c|c|c|c|}
\hline & FAMILY / Taxon & Life & Flor. element & Herb. & R.84 & R.90 & XK & EUNIS code & EU & END & Note \\
\hline 157. & Melica ciliata L. & $\mathrm{H}$ & Euri-Medit. & L-085 & $\bullet$ & & & $\mathrm{E} 1.2, \mathrm{E} 1.22, \mathrm{H} 2.6 \mathrm{~B}$ & & & \\
\hline 158. & Melica uniflora Retz. & $\mathrm{H}$ & Paleotemp. & L-483 & $\bullet$ & & & F3.2421, G1.A1 & & & \\
\hline 159. & Milium effusum L. & G & Circumbor. & L-484 & $\bullet$ & & & F2.231, G1.6C4, H3.2 & & & \\
\hline 160. & Nardus stricta L. & $\mathrm{H}$ & Orof.S.Eur. & L-086 & & $\bullet$ & & $\begin{array}{l}\text { F2.2, E4.31, E3.4, E4.34, } \\
\text { D4.2 }\end{array}$ & & & \\
\hline 161. & Ochlopoa annua (L.) H. Scholz & $\mathrm{T}$ & Cosmopol. & N/A & $\bullet$ & & & E3.5 & & & \\
\hline 162. & Patzkea paniculata (L.) G. H. Loos & $\mathrm{H}$ & Medit.-Mont. & N/A & & $\bullet$ & & E4.39, E4.4 & & & \\
\hline 163. & Phleum alpinum L. & $\mathrm{H}$ & Orof.S.Eur. & L-087 & $\bullet$ & $\bullet$ & & E1.7, E4.4, E4.41 & & & \\
\hline 164. & Phleum hirsutum Honck. & G & Orof.SE.Eur. & L-088 & & $\bullet$ & & $\mathrm{E} 1.2, \mathrm{E} 4.3$ & & & \\
\hline 165. & Phleum montanum Koch. & $\mathrm{H}$ & Euro-Siber. & L-089 & & & & $\mathrm{E} 4, \mathrm{E} 4.4$ & & & \\
\hline 166. & Phleum pratense $\mathrm{L}$. & $\mathrm{H}$ & Circumbor. & L-485 & $\bullet$ & & & $\mathrm{E} 1.2, \mathrm{E} 3.4$ & LC & & \\
\hline 167. & Poa alpina $\mathrm{L}$. & $\mathrm{H}$ & Circumbor. & L-090 & $\bullet$ & $\bullet$ & & $\mathrm{E} 1.22, \mathrm{E} 4.31, \mathrm{H} 2.4$ & LC & & \\
\hline 168. & Poa badensis Haenke & $\mathrm{H}$ & Orof.Europ. & L-092 & $\bullet$ & & & E1.2, E4.3, E4.39, H2.4 & & & \\
\hline 169. & Poa bulbosa L. & $\mathrm{H}$ & Paleotemp. & L-093 & $\bullet$ & & & $\mathrm{E} 1.2, \mathrm{E} 1.22, \mathrm{E} 1.112$ & & & \\
\hline 170. & Poa cenisia All. & G & Europ. & N/A & $\bullet$ & & & E4.1, E4.116, E4.12 & & & \\
\hline 171. & Poa chaixii Vill. & $\mathrm{H}$ & Euro-Cauc. & N/A & & $\bullet$ & & E4, E4.4126 & & & \\
\hline 172. & Poa media Schur & $\mathrm{H}$ & Balkan. & N/A & & $\bullet$ & & E3.52, E3.4 & & & \\
\hline 173. & Poa nemoralis L. & $\mathrm{H}$ & Circumbor. & N/A & $\bullet$ & & & E1.2, F3.2421 & & & \\
\hline 174. & Poa pratensis $\mathrm{L}$. & $\mathrm{H}$ & Circumbor. & L-486 & $\bullet$ & & & E1.22, E3.4, E3.3 & LC & & \\
\hline 175. & Poa trivialis subsp. sylvicola (Guss.) H. Lindb. & $\mathrm{H}$ & Euri-Medit. & N/A & $\bullet$ & & & E3.4 & & & \\
\hline 176. & Schedonorus pratensis (Huds.) P. Beauv. & $\mathrm{H}$ & Euro-Asiat. & N/A & $\bullet$ & & & E1.73 & & & \\
\hline 177. & Sesleria autumnalis (Scop.) F. W. Schultz & $\mathrm{H}$ & Orof.SE-Eur. & L-666 & $\bullet$ & & & F3.2421 & & & \\
\hline 178. & Sesleria coerulans Friv. & $\mathrm{H}$ & Balkan. & N/A & $\bullet$ & & & $\mathrm{E} 4.3, \mathrm{E} 4.39$ & & & \\
\hline 179. & Sesleria comosa Velen. & $\mathrm{H}$ & Balkan. & L-487 & $\bullet$ & $\bullet$ & & $\mathrm{E} 4.3, \mathrm{E} 4.31, \mathrm{E} 4.39$ & & End(B) & \\
\hline 180. & Sesleria juncifolia Suffren. & $\mathrm{H}$ & Anfriadriat. & L-095 & $\bullet$ & $\bullet$ & & E4.41, E4.4126, H2.4 & & & \\
\hline 181. & Sesleria korabensis (Kümmerle \& Jáv.) Deyl & $\mathrm{H}$ & Balkan & N/A & & $\bullet$ & & E4.3925, E4.413A, E4.3 & & End(B) & \\
\hline 182. & Sesleria latifolia (Adamović) Degen & $\mathrm{H}$ & Ilyrian-Apen. & L-096 & $\bullet$ & & & $\begin{array}{l}\mathrm{H} 2.6 \mathrm{~B}, \mathrm{E} 4.3925, \mathrm{H} 2.4 \text {, } \\
\mathrm{E} 1.112\end{array}$ & & & \\
\hline \multirow[t]{2}{*}{183.} & Sesleria wettsteinii Dörf \& Hayek. & $\mathrm{H}$ & Balkan & L-098 & & $\bullet$ & & $\mathrm{E} 4.41, \mathrm{E} 4.412$ & & End(B) & $\begin{array}{c}\text { CONS. } \\
+\end{array}$ \\
\hline & RANUNCULACEAE & & & & & & & & & & \\
\hline 184. & Actaea spicata L. & G & Euro-Asiat. & L-201 & $\bullet$ & & & G1.6C4 & & & \\
\hline 185. & Adonis flammea Jacq. & $\mathrm{T}$ & Euro-Asiat. & N/A & $\bullet$ & & & G1.7 & & & \\
\hline 186. & Anemonastrum narcissiflorum (L.) Holub & G & Euro-Asiat. & L-202 & $\bullet$ & $\bullet$ & & E4.3, E4.39, E4.392 & & & \\
\hline 187. & Anemone nemorosa $\mathrm{L}$. & G & Circumbor. & L-203 & $\bullet$ & $\bullet$ & & G1.6C4, E5.2, G3.1 & & & \\
\hline 188. & Anemone ranunculoides $\mathrm{L}$. & G & Euro-Asiat. & L-204 & $\bullet$ & & & G1.6C4 & & & \\
\hline
\end{tabular}


Annex. 1 - cont.

\begin{tabular}{|c|c|c|c|c|c|c|c|c|c|c|c|}
\hline & FAMILY / Taxon & Life & Flor. element & Herb. & R.84 & R.90 & $\mathrm{XK}$ & EUNIS code & EU & END & Note \\
\hline 189. & Caltha palustris L. & $\mathrm{H}$ & Circumbor. & L-205 & - & & & $\begin{array}{l}\text { D4.1, E3.4, D2.3, D2.38, } \\
\text { D2.26 }\end{array}$ & LC & & \\
\hline 190. & Clematis vitalba $\mathrm{L}$. & $\mathrm{P}$ & Euro-Asiat. & L-493 & - & & & F3.2421, G1.A1 & & & \\
\hline 191. & Consolida regalis Gray & $\mathrm{T}$ & Euro-Asiat. & $\mathrm{L}-488$ & - & & & E3.3 & & & \\
\hline 192. & Ficaria verna Huds. & G & Euro-Asiat. & L-206 & - & & & G1.6933, G1.A1 & & & \\
\hline 193. & Helleborus odorus Waldst. \& Kit. ex Willd. & G & Orof.SE-Eur. & L-207 & - & & & E1.2, F3.2421, G1.A1 & & & \\
\hline 194. & Isopyrum thalictroides $\mathrm{L}$. & G & Euro-Asiat. & L-208 & - & & & G1.7C1 & & & \\
\hline 195. & Nigella arvensis $\mathrm{L}$. & $\mathrm{T}$ & Euro-Asiat. & L-687 & • & & & E1.2 & & & \\
\hline 196. & Ranunculus acris L. & $\mathrm{H}$ & Euro-Siber. & L-489 & - & & & E4.4125, E3.3 & & & \\
\hline 197. & Ranunculus arvensis $\mathrm{L}$. & $\mathrm{T}$ & Euro-Asiat. & L-490 & - & & & G3.17 & & & \\
\hline 198. & Ranunculus breyninus Crantz & $\mathrm{H}$ & Orof.S.Eur. & L-209 & & & & E3.5 & & & \\
\hline 199. & Ranunculus bulbosus L. & $\mathrm{H}$ & Euro-Asiat. & N/A & - & & & E1.22 & & & \\
\hline 200. & Ranunculus crenatus Waldst. \& Kit. & $\mathrm{H}$ & Orof.S.Eur. & L-688 & - & & & E4.1, E4.116, E4.5 & & & \\
\hline 201. & Ranunculus fontanus C. Presl & $\mathrm{T}$ & Medit.-Mont. & L-725 & & & & D2.2 & DD & & $\begin{array}{c}\text { CONS. } \\
+\end{array}$ \\
\hline 202. & Ranunculus millefoliatus Vahl & $\mathrm{H}$ & Euri-Medit. & N/A & - & & & G1.7 & & & \\
\hline 203. & Ranunculus montanus Willd. & $\mathrm{H}$ & Orof.S.Eur. & L-210 & - & - & & $\begin{array}{l}\mathrm{F} 2.2, \mathrm{G} 1.7, \mathrm{E} 1.2, \mathrm{D} 2.38, \\
\mathrm{D} 2.26\end{array}$ & & & \\
\hline 204. & Ranunculus platanifolius L & $\mathrm{H}$ & Europ. & L-212 & - & & & G1.6C4, E3.5 & & & \\
\hline 205. & Ranunculus polyanthemos $\mathrm{L}$. & $\mathrm{H}$ & Euro-Asiat. & N/A & - & & & E3.5, E4 & & & \\
\hline 206. & Ranunculus repens L. & $\mathrm{H}$ & Paleotemp. & L-492 & - & & & E3.5, E4.4121, E3.3 & LC & & \\
\hline 207. & Ranunculus sardous Crantz & $\mathrm{T}$ & Euro-Asiat. & L-494 & - & & & E3.3, E3.4 & & & \\
\hline 208. & Thalictrum aquilegifolium L. & $\mathrm{H}$ & Euro-Siber. & L-213 & - & - & & E1.2, F3.2421, G1.7 & & & \\
\hline 209. & Thalictrum minus L. & $\mathrm{H}$ & Euro-Asiat. & L-214 & & & & $\mathrm{E} 1.2, \mathrm{E} 1.22, \mathrm{H} 2.3$ & & & \\
\hline 210. & Trollius europaeus L. & $\mathrm{H}$ & Circumbor. & L-215 & - & & LC & E3.5, E4.1, E4.5 & & & \\
\hline & PAPAVERACEAE & & & & & & & & & & \\
\hline 211. & Chelidonium majus L. & $\mathrm{H}$ & Euro-Asiat. & L-495 & - & & & G1.A1 & & & \\
\hline 212. & Corydalis cava (L.) Schweigg. \& Körte & G & Euro-Asiat. & L-105 & - & & & G1.6C4, G1.A1 & & & \\
\hline 213. & Corydalis solida (L.) Claivr. & G & Euro-Asiat. & L-106 & - & & & $\begin{array}{l}\text { G1.6C4, G1.6924, } \\
\text { G1.A1 }\end{array}$ & & & \\
\hline 214. & Papaver rhoeas $\mathrm{L}$. & $\mathrm{T}$ & Paleotemp. & L-496 & - & & & G1.7 & & & \\
\hline 215. & $\begin{array}{l}\text { Pseudofumaria alba (Mill.) Lidén subsp. alba } \\
\text { CRASSULACEAE }\end{array}$ & $\mathrm{H}$ & Anfriadriat. & L-107 & & & & $\mathrm{H} 2.6, \mathrm{H} 2.6 \mathrm{~B}$ & & & \\
\hline 216. & Jovibarba heuffelii (Schott) Á. Löve \& D. Löve & c & Balkan & L-114 & - & - & LC & E1.22, E4.4 & & & \\
\hline 217. & Sedum acre L. & c & Euro-Siber. & L-115 & $\bullet$ & & & $\begin{array}{l}\text { E1.2, E4.41, E4.4121, } \\
\text { E4.34 }\end{array}$ & & & \\
\hline 218. & Sedum album L. & C & Euro-Asiat. & L-116 & & & & $\mathrm{E} 1.2, \mathrm{E} 1.22, \mathrm{H} 2.4$ & & & \\
\hline
\end{tabular}


Annex. 1 - cont.

\begin{tabular}{|c|c|c|c|c|c|c|c|c|c|c|c|}
\hline & FAMILY / Taxon & Life & Flor. element & Herb. & R.84 & R.90 & $\mathrm{XK}$ & EUNIS code & EU & END & Note \\
\hline 219. & Sedum annuum $\mathrm{L}$. & $\mathrm{T}$ & Arctic-Alp. & L-665 & $\bullet$ & $\bullet$ & & $\mathrm{E} 1.112, \mathrm{H} 3.2$ & & & \\
\hline 220. & Sedum atratum $\mathrm{L}$. & $\mathrm{T}$ & Europ. & L-117 & & • & & E1.2, E1.22, H3.216 & & & \\
\hline 221. & Sedum cepaea L. & $\mathrm{T}$ & Euri-Medit. & N/A & • & & & H3.216 & & & \\
\hline 222. & Sedum grisebachii subsp. flexuosum (Wettst.) Greut. \& Burd. & $\mathrm{H}$ & Balkan. & L-689 & • & - & & H3.1, H3.152 & & & \\
\hline 223. & Sedum hispanicum L. & $\mathrm{H}$ & Euro-Asiat. & L-118 & • & • & & G1.7, G1.6924 & & & \\
\hline 224. & Sedum magellense Ten. & C & Euri-Medit. & N/A & - & & & $\mathrm{G} 1.6 \mathrm{C} 4, \mathrm{H} 2.6$ & & & \\
\hline 225. & Sedum maximum (L.) Holub. & $\mathrm{H}$ & Euro-Siber. & N/A & - & & & $F 3.2421$ & & & \\
\hline 226. & Sedum ochroleucum Chaix. & $\mathrm{H}$ & Euri-Medit. & L-119 & - & $\bullet$ & & $\begin{array}{l}\text { E1.2, E1.22, F3.2421, } \\
\text { H2.4 }\end{array}$ & & & \\
\hline 227. & Sempervivum erythraeum Velen. & C & Balkan & N/A & & - & & E1.112 & & & \\
\hline 228. & $\begin{array}{l}\text { Sempervivum macedonicum Praeger } \\
\text { SAXIFRAGACEAE }\end{array}$ & c & Balkan & L-120 & & & LC & E1.7, E1.112 & & End(B) & \\
\hline 229. & Chrysosplenium alternifolium L. & $\mathrm{H}$ & Euro-Siber. & N/A & - & & & G1.6933 & & & \\
\hline 230. & Saxifraga adscendens $\mathrm{L}$. & $\mathrm{H}$ & Orof.S.Eur. & L-121 & & - & & $\begin{array}{l}F 2.3, F 2.231, H 2.33, \\
H 2.4\end{array}$ & & & \\
\hline 231. & Saxifraga aizoides L. & $\mathrm{H}$ & Circumbor. & L-122 & & - & & $\begin{array}{l}\text { D4.1, E4.4, E4.41, } \\
\text { D2.38, D2.26 }\end{array}$ & & & \\
\hline 232. & Saxifraga bulbifera $\mathrm{L}$. & $\mathrm{H}$ & Euri-Medit. & N/A & - & & & G1.7 & & & \\
\hline 233. & Saxifraga exarata Vill. & $\mathrm{H}$ & Orof.SE-Eur. & N/A & - & & & H2.6 & & & \\
\hline 234. & Saxifraga marginata Sternb. & c & Orof.SE.Eur. & L-124 & & & & $\mathrm{H} 3.2, \mathrm{H} 2.6, \mathrm{H} 2.4$ & & & \\
\hline 235. & Saxifraga moschata Wulfen. & $\mathrm{H}$ & Euro-Asiat. & L-125 & - & - & & H3.1, H3.152, E1.112 & & & \\
\hline 236. & Saxifraga paniculata Mill. & $\mathrm{H}$ & Arctic-Alp. & L-126 & - & & & $\begin{array}{l}\text { G1.6922, H3.2, H3.152, } \\
\text { H2.4 }\end{array}$ & & & \\
\hline 237. & Saxifraga rotundifolia $\mathrm{L}$. & $\mathrm{H}$ & Europ. & L-127 & - & & & D4.1, H2.33, F3.24311 & & & \\
\hline 238. & Saxifraga scardica Griseb. & C & Balkan & $\mathrm{L}-128$ & - & - & LC & $\mathrm{H} 2.6, \mathrm{H} 2.6 \mathrm{~B}, \mathrm{H} 2.4$ & & $\operatorname{End}(B)$ & \\
\hline 239. & Saxifraga sempervivum Koch. & C & Balkan & L-129 & - & - & LC & $\begin{array}{l}\text { E4.41, E4.412, F2.1, } \\
\text { H2.4, E1.112 }\end{array}$ & & & \\
\hline 240. & Saxifraga taygetea Boiss. \& Heldr. & $\mathrm{H}$ & Balkan & L-130 & - & & EN & H3.1, H3.152 & & End(B) & \\
\hline 241. & $\begin{array}{l}\text { Saxifraga tridactylites } \mathrm{L} \text {. } \\
\text { VITACEAE }\end{array}$ & $\mathrm{T}$ & Euro-Asiat. & L-131 & & & & $\mathrm{H} 3.2, \mathrm{H} 3.216$ & & & \\
\hline 242. & $\begin{array}{l}\text { Vitis vinifera } \mathrm{L} \text {. } \\
\text { PARNASSIACEAE }\end{array}$ & $\mathrm{P}$ & Euro-Asiat. & L-497 & - & & & G1.6C4, G1.6933 & LC & & \\
\hline 243. & Parnassia palustris L. & $\mathrm{H}$ & Euro-Siber. & L-033 & & & & $\begin{array}{l}\mathrm{D} 2.2, \mathrm{D} 2.22, \mathrm{D} 2.3 \\
\mathrm{D} 2.38, \mathrm{D} 2.26\end{array}$ & & & \\
\hline 244 & $\begin{array}{l}\text { CELASTRACEAE } \\
\text { Eunvmus eurongeus }\end{array}$ & $P$ & Furo-Asiat & $1-500$ & - & & & $G 17 E_{3} 2 G_{3} 1 \mathrm{G} 1 \mathrm{~A} 1$ & & & \\
\hline
\end{tabular}


Annex. 1 - cont.

\begin{tabular}{|c|c|c|c|c|c|c|c|c|c|c|c|}
\hline & FAMILY / Taxon & Life & Flor. element & Herb. & R.84 & R.90 & $\mathrm{XK}$ & EUNIS code & EU & END & Note \\
\hline \multirow[t]{2}{*}{245.} & Euonymus verrucosus Scop. & $P$ & Europ. & L-501 & $\bullet$ & & & $\begin{array}{l}\text { G1.6933, F3.2421, } \\
\text { G1.6C4, G1.A1 }\end{array}$ & & & \\
\hline & OXALIDACEAE & & & & & & & & & & \\
\hline \multirow[t]{2}{*}{246.} & Oxalis acetosella $\mathrm{L}$. & $\mathrm{C}$ & Circumbor. & L-099 & $\bullet$ & & & $\begin{array}{l}\text { G1.6933, G1.7, E5.2, } \\
\text { G3.1 }\end{array}$ & & & \\
\hline & CLUSIACEAE & & & & & & & & & & \\
\hline 247. & Hypericum barbatum Jacq. & $\mathrm{H}$ & Europ. & L-498 & $\bullet$ & & & E1.2, E1.55, E4.4 & & & \\
\hline 248. & Hypericum maculatum Crantz & $\mathrm{H}$ & Euro-Asiat. & L-499 & $\bullet$ & $\bullet$ & & $\begin{array}{l}\text { E1.7, E4.3, E4.31, } \\
\text { E4.312 }\end{array}$ & & & \\
\hline 249. & Hypericum montanum L. & $\mathrm{H}$ & Euro-Asiat. & L-101 & & & & $\begin{array}{l}\text { E4.3, E4.39, E4.392, } \\
\text { E5.2 }\end{array}$ & & & \\
\hline 250. & Hypericum perforatum subsp. Iatifolium (Gaudin) A. Frohl. & $\mathrm{H}$ & Paleotemp. & L-102 & $\bullet$ & & & $\begin{array}{l}\text { E1.2, E1.22, E1.7, E5.2, } \\
\text { E4.34 }\end{array}$ & & & \\
\hline \multirow[t]{2}{*}{251.} & Hypericum richeri subsp. grisebachii (Boiss.) Nyman & $\mathrm{H}$ & Europ. & L-103 & $\bullet$ & $\bullet$ & & $\begin{array}{l}\mathrm{E} 4.3, \mathrm{E} 4.31, \mathrm{E} 4.312, \\
\mathrm{E} 4.39\end{array}$ & & & \\
\hline & SALICACEAE & & & & & & & & & & \\
\hline 252. & Populus alba L. & $\mathrm{P}$ & Euro-Asiat. & L-502 & $\bullet$ & & & G1.7 & & & \\
\hline 253. & Populus tremula & $P$ & Circumtemp. & L-132 & $\bullet$ & & & $\begin{array}{l}\text { F3.2421, G1.6922, } \\
\text { G1.6C4, E5.2 }\end{array}$ & & & \\
\hline 254. & Salix alba L. & $\mathrm{P}$ & Euro-Asiat. & L-503 & $\bullet$ & & & F3.2421, G1.1 & & & \\
\hline 255. & Salix caprea L. & $\mathrm{P}$ & Euro-Siber. & L-133 & & & & $\begin{array}{l}\text { G1.6933, G3.12, G4.6, } \\
\text { E5.2 }\end{array}$ & & & \\
\hline 256. & Salix reticulata $\mathrm{L}$. & $\mathrm{C}$ & Circumbor. & L-134 & $\bullet$ & & LC & $\begin{array}{l}\text { E1.7, E4.41, E4.4125, } \\
\text { F2.12 }\end{array}$ & & & \\
\hline \multirow[t]{2}{*}{257.} & Salix retusa L. & C & Orof.Europ. & L-135 & $\bullet$ & $\bullet$ & & $\begin{array}{l}\text { E4.4, E4.412, F2.1, } \\
\text { F2.12 }\end{array}$ & & & \\
\hline & VIOLACEAE & & & & & & & & & & \\
\hline 258. & Viola elegantula Schott. & $\mathrm{H}$ & Ballkan & L-109 & & & LC & $\begin{array}{l}\text { E1.73, E4.3922, } \\
\text { E4.4122 }\end{array}$ & & End(B) & \\
\hline 259. & Viola grisebachiana Vis. & $\mathrm{H}$ & Ballkan & L-111 & & & EN & $\mathrm{H} 2.6, \mathrm{H} 2.6 \mathrm{~B}$ & & End(B) & \\
\hline 260. & Viola jordanii Hanry & $\mathrm{H}$ & Euro-Asiat. & L-690 & $\bullet$ & & & G1.6C4 & & & \\
\hline 261. & Viola kitaibeliana Schult. & $\mathrm{T}$ & Euri-Medit. & N/A & $\bullet$ & & & $\mathrm{E} 1.2, \mathrm{E} 1.22$ & & & \\
\hline 262. & Viola odorata $\mathrm{L}$. & $\mathrm{H}$ & Euro-Asiat. & L-112 & $\bullet$ & & & G1.7, G1.6C4, G1.A1 & & & \\
\hline 263. & Viola orphanidis Boiss. & $\mathrm{H}$ & Ballkan & L-110 & $\bullet$ & & LC & $\begin{array}{l}\text { E5.5721, E4.413A, } \\
\text { G3.12 }\end{array}$ & & End(B) & \\
\hline 264. & Viola reichenbachiana Boreau & $\mathrm{H}$ & Euro-Asiat. & N/A & $\bullet$ & & & G1.6C4 & & & \\
\hline 265. & Viola schariensis Erben & $\mathrm{H}$ & Ballkan & L-113 & & & & E4.41, E4.4122 & & End(B) & $\nabla$ \\
\hline 266. & Viola tricolor L. subsp. tricolor & $\mathrm{T}$ & Euro-Asiat. & L-104 & $\bullet$ & & & $\mathrm{E} 1.74, \mathrm{~F} 2.23, \mathrm{G} 1.7$ & & & \\
\hline
\end{tabular}


Annex. 1 - cont.

\begin{tabular}{|c|c|c|c|c|c|c|c|c|c|c|c|}
\hline & FAMILY / Taxon & Life & Flor. element & Herb. & R.84 & R.90 & XK & EUNIS code & EU & END & Note \\
\hline 267. & $\begin{array}{l}\text { Viola tricolor subsp. macedonica (Boiss. \& Heldr.) A. F. W. } \\
\text { Schmidt } \\
\text { LINACEAE }\end{array}$ & $\mathrm{T}$ & Balkan & L-504 & & $\bullet$ & & E1.7 & & End(B) & \\
\hline 268. & Linum capitatum Kit. ex Schult. & $\mathrm{C}$ & Orof.SE.Eur. & L-099 & $\bullet$ & $\bullet$ & & $\begin{array}{l}\text { E4.3, E4.31, E4.39, } \\
\text { E4.34 }\end{array}$ & & End(B) & \\
\hline 269. & Linum catharticum L. & $\mathrm{T}$ & Euri-Medit. & N/A & $\bullet$ & & & $\mathrm{E} 1.2, \mathrm{E} 1.22, \mathrm{E} 1.73$ & & & \\
\hline 270. & Linum flavum $\mathrm{L}$. & $\mathrm{H}$ & Europ. & L-505 & $\bullet$ & & LC & $\mathrm{E} 1.2, \mathrm{E} 1.22$ & & & \\
\hline 271. & Linum hologynum Rchb. & $\mathrm{H}$ & Balkan & N/A & $\bullet$ & & & E1.2, E5.4 & & & \\
\hline 272. & Linum perenne L. & $\mathrm{H}$ & Euri-Medit. & L-663 & $\bullet$ & & & $\mathrm{E} 1.2, \mathrm{E} 1.74$ & & & \\
\hline 273. & $\begin{array}{l}\text { Linum tenuifolium L. } \\
\text { EUPHORBIACEAE }\end{array}$ & $\mathrm{H}$ & Euro-Asiat. & L-664 & $\bullet$ & & & E1.2, E4.392 & & & \\
\hline 274. & Euphorbia amygdaloides L. & $\mathrm{C}$ & Euro-Cauc. & L-136 & $\bullet$ & & & $\begin{array}{l}\text { E4.412, F2.23 E5.2, } \\
\text { G3.1, G1.A1 }\end{array}$ & & & \\
\hline 275. & Euphorbia cyparissias L. & $\mathrm{H}$ & Centro-Eur. & L-137 & $\bullet$ & & & $\mathrm{E} 1.22, \mathrm{E} 1.7$ & & & \\
\hline 276. & Euphorbia epithymoides L. & $\mathrm{H}$ & Europ. & N/A & $\bullet$ & & & $\mathrm{G} 1.7 \mathrm{C} 1$ & & & \\
\hline 277. & Euphorbia glabriflora Vis. & $\mathrm{C}$ & Balkan & L-662 & $\bullet$ & & & $\mathrm{E} 1.2, \mathrm{~F} 3.2421$ & & End(B) & \\
\hline 278. & Euphorbia helioscopia L. & $\mathrm{T}$ & Cosmopol. & L-506 & $\bullet$ & & & $\mathrm{E} 1.2$ & & & \\
\hline 279. & Euphorbia myrsinites L. & $\mathrm{H}$ & Euri-Medit. & L-507 & $\bullet$ & & & $\mathrm{E} 1.2, \mathrm{G} 1.7$ & & & \\
\hline 280. & $\begin{array}{l}\text { Mercurialis perennis } \mathrm{L} \text {. } \\
\text { FABACEAE }\end{array}$ & G & Euro-Asiat. & L-508 & $\bullet$ & & & E4.4, G1.6C4, G1.A1 & & & \\
\hline 281. & Anthyllis aurea Welden. & $\mathrm{H}$ & Orof.SE.Eur. & $\mathrm{L}-253$ & & & & E1.22, E4.4, E4.4125 & & End(B) & \\
\hline 282. & Anthyllis montana $\mathrm{L}$. & $\mathrm{C}$ & Medit. & L-509 & $\bullet$ & & & $\begin{array}{l}\text { E4.4126, E4.41731, } \\
\text { E1.112 }\end{array}$ & & & \\
\hline 283. & Anthyllis vulneraria subsp. alpestris (Schult.) Asch. \& Graebn. & $\mathrm{H}$ & Balkan & L-255 & & $\bullet$ & & E4.41731 & & & \\
\hline 284. & Astragalus glycyphyllos L. & $\mathrm{H}$ & Euro-Siber. & N/A & $\bullet$ & & & F3.2421, G1.7 & & & \\
\hline 285. & Astragalus onobrychis L. & $\mathrm{H}$ & Euro-Asiat. & L-510 & $\bullet$ & & & E1.112 & & & \\
\hline 286. & Colutea arborescens L. & $\mathrm{P}$ & Euro-Asiat. & L-511 & $\bullet$ & & & F3.2421, G1.7 & & & \\
\hline 287. & Cytisus albus Hacq. & $\mathrm{C}$ & Balkan & $\mathrm{L}-258$ & $\bullet$ & $\bullet$ & & $F 2.2, F 3.2421$ & & & \\
\hline 288. & Cytisus hirsutus L. & $\mathrm{C}$ & Euro-Siber. & $\mathrm{L}-256$ & $\bullet$ & & & $\mathrm{F} 2.3, \mathrm{~F} 2.32$ & & & \\
\hline 289. & Cytisus procumbens (Willd.) Spreng. & $\mathrm{C}$ & Balkan & L-257 & $\bullet$ & & & $F 2.2, F 2.23$ & & & \\
\hline 290. & Genista depressa M. Bieb. & $\mathrm{C}$ & Balkan & L-259 & & & & $\mathrm{E} 1.2, \mathrm{E} 1.7$ & & & $\nabla$ \\
\hline 291. & Genista sagittalis L. & $\mathrm{C}$ & Europ. & L-512 & $\bullet$ & & & $\mathrm{E} 1.2, \mathrm{E} 1.22, \mathrm{E} 1.7$ & & & \\
\hline 292. & Genista tinctoria L. & $\mathrm{C}$ & Euro-Siber. & L-260 & $\bullet$ & & & $\mathrm{E} 1.2, \mathrm{G} 1.6 \mathrm{C} 4$ & & & \\
\hline 293. & Hippocrepis comosa $\mathrm{L}$. & $\mathrm{H}$ & Europ. & L-100 & $\bullet$ & & & E1.2, E4.392 & & & \\
\hline 294. & Hippocrepis glauca Ten. & $\mathrm{H}$ & Euri-Medit. & $\mathrm{L}-221$ & & $\bullet$ & & $\mathrm{E} 1.2$ & & & \\
\hline 295. & Lathyrus aphaca L. & $\mathrm{T}$ & Euri-Medit. & N/A & $\bullet$ & $\bullet$ & & G1.7, G1.A1 & & & \\
\hline 296. & Lathyrus latifolius L. & $\mathrm{H}$ & Euri-Medit. & L-123 & $\bullet$ & & & E1.7, E1.74 & $\mathrm{LC}$ & & \\
\hline
\end{tabular}


Annex. 1 - cont.

\begin{tabular}{|c|c|c|c|c|c|c|c|c|c|c|c|}
\hline & FAMILY / Taxon & Life & Flor. element & Herb. & R.84 & R.90 & $\mathrm{XK}$ & EUNIS code & EU & END & Note \\
\hline 297. & Lathyrus niger (L.) Bernh. & $\mathrm{H}$ & Euri-Medit. & L-513 & - & & & G1.6924, G1.7 & & & \\
\hline 298. & Lathyrus pratensis L. & $\mathrm{H}$ & Paleotemp. & N/A & - & & & $\mathrm{E} 1.2, \mathrm{E} 1.73$ & & & \\
\hline 299. & Lathyrus tuberosus L. & $\mathrm{H}$ & Euro-Siber. & L-661 & - & & & E3.4 & LC & & \\
\hline 300. & Lembotropis nigricans (L.) Griseb. & $\mathrm{P}$ & Orof.S.Eur. & N/A & - & & & F3.242, G1.7 & & & \\
\hline 301. & Lotus corniculatus L. & $\mathrm{H}$ & Euro-Asiat. & L-222 & • & - & & E1.22, E1.7, E5.2, E3.3 & LC & & \\
\hline 302. & Lotus tenuis Willd. & $\mathrm{H}$ & Euro-Asiat. & N/A & - & & & E3.4 & & & \\
\hline 303. & Medicago prostrata Jacq. & $\mathrm{H}$ & Orof.S.Eur. & L-514 & - & & & E1.2, E1.7, E1.22 & LC & End(B) & \\
\hline 304. & Onobrychis montana subsp. scardica Griseb. & $\mathrm{H}$ & Balkan & L-224 & - & - & & $\begin{array}{l}\text { E4.4, E4.41, E4.412, } \\
\text { E1.112 }\end{array}$ & & End(B) & \\
\hline 305. & Ononis spinosa $\mathrm{L}$. & c & Euri-Medit. & L-660 & - & & & E3.4 & & & \\
\hline 306. & Oxytropis dinarica (Murb.) Wettst. & $\mathrm{H}$ & Balkan & L-703 & & - & & E4.4 & & End(B) & \\
\hline 307. & Oxytropis halleri subsp. korabensis (Küm. \& Jáv.) Chr. \& Ch. & $\mathrm{H}$ & Balkan & L-227 & & & & E4.4, E4.412 & & End(B) & \\
\hline 308. & Oxytropis jacquinii Bunge & c & Endem. Alp. & L-704 & & • & & E4.4, E4.42 & & & \\
\hline 309. & Robinia pseudoacacia L. & $\mathrm{P}$ & N. Amer. & L-515 & - & & & G1.7 & & & \\
\hline 310. & Trifolium alpestre L. & $\mathrm{H}$ & Euro-Cauc. & L-228 & - & - & & $\begin{array}{l}\text { E1.2, E1.22, E1.73, } \\
\text { E4.31 }\end{array}$ & LC & & \\
\hline 311. & Trifolium arvense L. & T & Paleotemp. & L-659 & • & & & E1.2, E1.22 & LC & & \\
\hline 312. & Trifolium badium Schreb. & $\mathrm{H}$ & Orof.S.Eur. & L-229 & • & & & $\mathrm{D} 2.3, \mathrm{D} 2.38$ & & & \\
\hline 313. & Trifolium campestre Schreb. & $\mathrm{T}$ & Paleotemp. & L-230 & - & & & $\begin{array}{l}\text { E1.2, E4.116, E4.391, } \\
\text { E5.2, E4.34 }\end{array}$ & & & \\
\hline 314. & Trifolium echinatum M. Bieb. & T & Euri-Medit. & L-516 & - & & & $\mathrm{E} 1.22, \mathrm{G} 1.7$ & & & \\
\hline 315. & Trifolium fragiferum $\mathrm{L}$. & $\mathrm{H}$ & Paleotemp. & N/A & - & & & E3.4, E3.3 & & & \\
\hline 316. & Trifolium hybridum L. & $\mathrm{H}$ & Euro-Asiat. & L-231 & & & & E3.4, E3.5 & LC & & \\
\hline 317. & Trifolium incarnatum L. & $\mathrm{T}$ & Euri-Medit. & L-517 & - & & & $\mathrm{E} 1.2, \mathrm{E} 1.22$ & LC & & \\
\hline 318. & Trifolium medium subsp. balcanicum Velen & G & Balkan & L-252 & • & & & $F 2.32$, E5.2 & & End(B) & \\
\hline 319. & Trifolium nigrescens Viv. & $\mathrm{T}$ & Euri-Medit. & N/A & - & & & E4.42 & LC & & \\
\hline 320. & Trifolium noricum Wulf. & $\mathrm{H}$ & Orof.SE.Eur. & L-233 & - & & & E4.4, E4.41, E4.412 & & & \\
\hline 321. & Trifolium patens Schreb. & T & Orof.S.Eur. & N/A & - & & & E1.2, E1.22, E3.4 & & & \\
\hline 322. & Trifolium pignantii Fauché \& Chaub. & G & Balkan & L-234 & - & & & F3.2421, G1.7, G1.A1 & & End(B) & \\
\hline 323. & Trifolium pratense L & $\mathrm{H}$ & Euro-Asiat. & L-235 & $\bullet$ & & & $\begin{array}{l}\text { E1.2, E1.22, E1.7, E1.73, } \\
\text { E5.2 }\end{array}$ & LC & & \\
\hline 324. & Trifolium repens $\mathrm{L}$. & $\mathrm{H}$ & Paleotemp. & L-136 & - & - & & $\begin{array}{l}\text { E4.4125, E5.5721, } \\
\text { E1.22, D2.38 }\end{array}$ & LC & & \\
\hline 325. & Trifolium resupinatum L. & T & Paleotemp. & L-518 & - & & & E3.4, E3.3 & LC & & \\
\hline 326. & Trifolium velenovskyi Vandas & $\mathrm{H}$ & Balkan & L-137 & - & & & E1.7, E1.73, E1.74 & & End(B) & \\
\hline 327. & Trifolium wettsteinii Dörfl. \& Hayek & c & Balkan & L-519 & - & & EN & E1.22, E4.4, E4.412 & & End(B) & \\
\hline
\end{tabular}


Annex. 1 - cont.

\begin{tabular}{|c|c|c|c|c|c|c|c|c|c|c|c|}
\hline & FAMILY / Taxon & Life & Flor. element & Herb. & R.84 & R.90 & XK & EUNIS code & EU & END & Note \\
\hline 328. & Polygala alpestris Reichenb. & $\mathrm{H}$ & Orof.S.Eur. & L-141 & & & & E4.4125 & & & \\
\hline 329. & Polygala comosa Schkuhr. & $\mathrm{H}$ & Orof.S.Eur. & L-142 & - & - & & $\mathrm{E} 1.2, \mathrm{E} 1.22$ & & & \\
\hline 330. & Polygala major Jacq. & $\mathrm{H}$ & Orof.S.Eur. & L-143 & - & & & E1.2, E1.22, E4.41 & & & \\
\hline 331. & $\begin{array}{l}\text { Polygala vulgaris } \mathrm{L} \text {. } \\
\text { ROSACEAE }\end{array}$ & $\mathrm{H}$ & Euro-Asiat. & L-144 & - & & & E1.2, E1.22, E1.7 & & & \\
\hline 332. & Agrimonia eupatoria L. & $\mathrm{H}$ & Subcosmop. & L-521 & - & & & E1.2, E1.7, E4.3925 & & & \\
\hline 333. & Alchemilla connivens Buser & $\mathrm{H}$ & Europ. & L-251 & - & & & E4.116, E4.5 & & & \\
\hline 334. & Alchemilla flabellata Buser & $\mathrm{H}$ & Euro-Asiat. & L-520 & - & - & & E4.31, E4.3925, E1.92 & & & \\
\hline 335. & Alchemilla glaucescens Wallr. & $\mathrm{H}$ & Europ. & L-303 & & & & $\begin{array}{l}\text { F2.231, E5.5721, D2.38, } \\
\text { E4.34 }\end{array}$ & & & \\
\hline 336. & Alchemilla velebitica Borb s ex Janch. & $\mathrm{H}$ & Balkan & L-305 & - & & & $\mathrm{H} 2.6 \mathrm{~B}, \mathrm{H} 2.6 \mathrm{~B} 3$ & & & \\
\hline 337. & Alchemilla viridiflora Rothm. & $\mathrm{H}$ & Balkan & L-306 & & & & D2.2, E5.5721 & & End(B) & \\
\hline 338. & Aremonia agrimonoides (L.) DC. & $\mathrm{H}$ & Orof.SE.Eur. & L-522 & - & - & & G1.6933, G3.1 & & & \\
\hline 339. & Aruncus dioicus (Walter) Fernald & $\mathrm{H}$ & Circumbor. & L-307 & - & & & G1.6933, E4.412 & & & \\
\hline 340. & Cotoneaster integerrimus Medik. & $\mathrm{P}$ & Euro-Asiat. & L-691 & - & & & $\begin{array}{l}\text { E1.2, E1.22, E4.4, } \\
\text { E4.4122 }\end{array}$ & & & \\
\hline 341. & Cotoneaster nebrodensis (Guss.) C. Koch. & $\mathrm{P}$ & Orof.S.Eur. & L-308 & & & & H3.152 & & & \\
\hline 342. & Crataegus monogyna Jacq. & $\mathrm{P}$ & Paleotemp. & L-523 & - & & & $\mathrm{E} 1.2, \mathrm{G} 1.7, \mathrm{G} 1 . \mathrm{A} 1$ & & & \\
\hline 343. & Crataegus pentagyna Willd. & $\mathrm{P}$ & Euro-Asiat. & L-692 & - & & & G3.12, F3.2421 & & & \\
\hline 344. & Dryas octopetala L. & c & Steno-Medit. & L-309 & - & - & & $\begin{array}{l}\text { E4.4, E4.41, H2.6, H3.1, } \\
\text { H2.4 }\end{array}$ & & & \\
\hline 345. & Filipendula ulmaria (L.) Maxim. & $\mathrm{H}$ & Euro-Siber. & L-310 & & & & E5.5, E5.51, E4.413A & & & \\
\hline 346. & Filipendula vulgaris Moench & $\mathrm{H}$ & Euro-Siber. & L-524 & - & & & E1.73 & & & \\
\hline 347. & Fragaria vesca $\mathrm{L}$. & $\mathrm{H}$ & Euro-Siber. & L-311 & $\bullet$ & & & $\begin{array}{l}\text { E1.22, E4.392, E5.2, } \\
\text { G3.1, G1.A1 }\end{array}$ & LC & & \\
\hline 348. & Geum coccineum Sibth. et.Sm. & $\mathrm{H}$ & Balkan & L-312 & - & - & & $\begin{array}{l}\text { E5.51, E5.5721, D2.38, } \\
\text { D2.26 }\end{array}$ & & & \\
\hline 349. & Geum montanum L. & $\mathrm{H}$ & Orof.S.Eur. & L-313 & - & $\bullet$ & & $\begin{array}{l}\text { E4.3, E4.31, E4.312, } \\
\text { E1.92, E4.34 }\end{array}$ & & & \\
\hline 350. & Geum urbanum L. & $\mathrm{H}$ & Circumbor. & L-314 & - & & & $\begin{array}{l}\text { E5.4, E5.5721, E5.2, } \\
\text { G1.A1 }\end{array}$ & & & \\
\hline 351. & Malus sy/vestris (L.) Mill. & $\mathrm{P}$ & Europ. & L-525 & - & & & G1.6933 & DD & & \\
\hline 352. & Potentilla aurea subsp. chrysocraspeda (Lehm.) Nyman & $\mathrm{H}$ & Balkan & L-316 & - & - & & E4.39 & & & \\
\hline 353. & Potentilla doerfleri Wettst. & $\mathrm{H}$ & Balkan & L-178 & & & EN & H3.15, H3.152 & & End(B) & \\
\hline 354. & Potentilla hirta $\mathrm{L}$. & $\mathrm{H}$ & Euri-Medit. & L-693 & - & & & E1.22, E1.7 & & & \\
\hline 355. & Potentilla inclinata Vill. & $\mathrm{H}$ & Euro-Asiat. & N/A & - & & & E1.2, E1.22 & & & \\
\hline 356. & Potentilla recta $\mathrm{L}$. & $\mathrm{H}$ & Euro-Siber. & L-658 & - & & & E1.2, F3.2421, G1.7 & & & \\
\hline
\end{tabular}


Annex. 1 - cont.

\begin{tabular}{|c|c|c|c|c|c|c|c|c|c|c|c|}
\hline & FAMILY / Taxon & Life & Flor. element & Herb. & R.84 & R.90 & $\mathrm{XK}$ & EUNIS code & EU & END & Note \\
\hline 357. & Potentilla reptans $\mathrm{L}$. & $\mathrm{H}$ & Paleotemp. & L-526 & $\bullet$ & & & D4.1, E3.4, E3.3 & & & \\
\hline 358. & Potentilla tommasiniana F. W. Schultz & $\mathrm{H}$ & Pontic & N/A & - & & & $\mathrm{E} 1.2, \mathrm{E} 1.22, \mathrm{G} 1.7 \mathrm{C1}$ & & & \\
\hline 359. & Prunus avium (L.) L. & $\mathrm{P}$ & Pontic & L-317 & - & & & $\begin{array}{l}\text { G1.6C4, G1.7C1, G4.6, } \\
\text { G1.A1 }\end{array}$ & LC & & \\
\hline 360. & Prunus spinosa $\mathrm{L}$. & $\mathrm{P}$ & Euro-Cauc. & L-527 & • & & & F3.242, G1.A1 & LC & & \\
\hline 361. & Pyrus communis subsp. pyraster (L.) Ehrh. & $\mathrm{P}$ & Euro-Asiat. & L-528 & - & & & F3.2421, G1.A1 & LC & & \\
\hline 362. & Rosa arvensis Huds. & $\mathrm{P}$ & Euri-Medit. & L-529 & - & & & G1.7, G1.A1 & & & \\
\hline 363. & Rosa canina L. & $\mathrm{P}$ & Paleotemp. & L-530 & • & & & E1.2, F3.2421 & & & \\
\hline 364. & Rosa gallica $\mathrm{L}$. & $\mathrm{P}$ & Euro-Asiat. & L-657 & - & & & G1.7 & & & \\
\hline 365. & Rosa glauca Pourret. & $\mathrm{P}$ & Orof.S.Eur. & L-318 & & & & G1.7, E4.34 & & & \\
\hline 366. & Rosa micrantha Sm. & $\mathrm{P}$ & Orof.S.Eur. & L-319 & - & & & $\mathrm{E} 4.41, \mathrm{E} 5.2$ & & & \\
\hline 367. & Rosa pendulina $\mathrm{L}$. & $\mathrm{P}$ & Orof.S.Eur. & L-320 & & - & & $\begin{array}{l}\text { G1.A1, G1.6C4, G1.7, } \\
\text { E5.3 }\end{array}$ & & & \\
\hline 368. & Rosa pulverulenta M. Bieb. & $\mathrm{P}$ & Medit.-Mont. & L-321 & & & & D2.282 & & & \\
\hline 369. & Rosa villosa $\mathrm{L}$. & $\mathrm{P}$ & Orof.S.Eur. & L-322 & & & & F2.22 & & & \\
\hline 370. & Rubus canescens DC. & $\mathrm{P}$ & Euro-Asiat. & L-323 & & & & G1.7 & & & \\
\hline 371. & Rubus idaeus $\mathrm{L}$. & $\mathrm{P}$ & Circumbor. & L-324 & - & - & & $\begin{array}{l}\text { G1.7, E1.92, E4.41, } \\
\text { H2.6, G3.1 }\end{array}$ & & & \\
\hline 372. & Sanguisorba minor Scop. & $\mathrm{H}$ & Euro-Asiat. & L-531 & - & & & E1.2, E1.22 & & & \\
\hline 373. & Sanguisorba officinalis L. & $\mathrm{H}$ & Circumbor. & L-532 & - & & & $\mathrm{D} 2.3, \mathrm{D} 2.38$ & & & \\
\hline 374. & Sorbus aria (L.) Crantz & $\mathrm{P}$ & Euri-Medit. & L-533 & • & & & E5.5, F3.2421 & & & \\
\hline 375. & Sorbus aucuparia L. & $\mathrm{P}$ & Euro-Asiat. & L-534 & - & & & G1.6933, G3.1 & & & \\
\hline 376. & Sorbus austriaca (Beck) Prain \& al. & $\mathrm{P}$ & Europ. & L-326 & & & & $F 2.1, F 2.2$ & & & \\
\hline 377. & Sorbus chamaemespilus (L.) Crantz & $\mathrm{P}$ & Orof.S.Eur. & N/A & & - & & $F 2.32, F 2.231$ & & & \\
\hline 378. & Sorbus domestica L. & $\mathrm{P}$ & Euri-Medit. & L-535 & - & & & G1.7 & & & \\
\hline 379. & Sorbus torminalis (L.) Crantz & $\mathrm{P}$ & Paleotemp. & L-536 & - & & & G1.7, G1.7C1, G1.A1 & & & \\
\hline 380. & $\begin{array}{l}\text { Waldsteinia geoides Willd. } \\
\text { MORACEAE }\end{array}$ & $\mathrm{H}$ & Euro-Asiat. & L-537 & - & & & G1.7, G4.6 & & & \\
\hline 381. & $\begin{array}{l}\text { Morus alba L. } \\
\text { RHAMNACEAE }\end{array}$ & $\mathrm{P}$ & Euro-Asiat. & L-694 & - & & & G1.1 & & & \\
\hline 382. & $\begin{array}{l}\text { Rhamnus alpina subsp. fallax (Boiss.) M. \& P. } \\
\text { ULMACEAE }\end{array}$ & $\mathrm{P}$ & Orof.SE.Eur. & L-160 & & - & & E4.412, G1.6933 & & & \\
\hline 383. & $\begin{array}{l}\text { Ulmus glabra Huds. } \\
\text { URTICACEAE }\end{array}$ & $\mathrm{P}$ & Euro-Cauc. & L-538 & - & & & G1.6933, G1.7, G1.A1 & & & \\
\hline 384. & Urtica dioica L. & $\mathrm{H}$ & Subcosmop. & L-150 & - & - & & $\begin{array}{l}\text { E5.5721, E4.41731, } \\
\text { E5.2, G1.1 }\end{array}$ & LC & & \\
\hline 385. & Urtica urens $\mathrm{L}$. & $T$ & Subcosmop. & N/A & - & & & $\mathrm{G} 1.7$ & & & \\
\hline
\end{tabular}


Annex. 1 - cont.

\begin{tabular}{|c|c|c|c|c|c|c|c|c|c|c|c|}
\hline & FAMILY / Taxon & Life & Flor. element & Herb. & R.84 & R.90 & $\mathrm{XK}$ & EUNIS code & EU & END & Note \\
\hline & GERANIACEAE & & & & & & & & & & \\
\hline 386. & Erodium cicutarium (L.) L'Hér. & $\mathrm{T}$ & Subcosmop. & L-539 & - & & & G1.7, E1.92 & & & \\
\hline 387. & Geranium lucidum L. & T & Euri-Medit. & L-656 & - & & & $\mathrm{H} 2.6, \mathrm{H} 2.6 \mathrm{~B}, \mathrm{H} 2.6 \mathrm{~B} 3$ & & & \\
\hline 388. & Geranium macrorrhizum L. & G & Orof.SE.Eur. & L-145 & - & - & & E1.2, E1.22, F2.2, F2.23 & & & \\
\hline 389. & Geranium molle L. & $\mathrm{T}$ & Euro-Asiat. & N/A & - & & & $\mathrm{E} 1.2, \mathrm{E} 1.22$ & & & \\
\hline 390. & Geranium robertianum L. & $\mathrm{T}$ & Subcosmop. & L-146 & - & & & $\begin{array}{l}\text { E5.43, G1.6933, E5.2, } \\
\text { G1.A1 }\end{array}$ & & & \\
\hline 391. & Geranium subcaulescens DC. & $\mathrm{H}$ & Orof.SE.Eur. & L-179 & & & LC & E4.3, E4.31, E4.39, F2.2 & & & \\
\hline 392. & $\begin{array}{l}\text { Geranium sylvaticum L. } \\
\text { FAGACEAE }\end{array}$ & $\mathrm{H}$ & Euro-Asiat. & L-147 & - & - & & F2.32, G1.6933 & & & \\
\hline 393. & Fagus sylvatica $\mathrm{L}$. & $\mathrm{P}$ & Centro-Eur. & L-138 & - & & & $\begin{array}{l}\text { G1.6933, G1.6C4, E5.2, } \\
\text { G3.1 }\end{array}$ & & & \\
\hline 394. & Quercus cerris L. & $\mathrm{P}$ & Euri-Medit. & L-540 & - & & & $\begin{array}{l}\text { G1.7, F3.2421, G1.69, } \\
\text { G1.A1 }\end{array}$ & & & \\
\hline 395. & Quercus frainetto Ten. & $\mathrm{P}$ & Orof.SE-Eur. & L-541 & - & & & G1.7, G1.A1 & & & \\
\hline 396. & Quercus petraea (Matt.) Liebl. & $\mathrm{P}$ & Europ. & L-542 & - & & & G1.6933, F3.242, G1.A1 & & & \\
\hline 397. & $\begin{array}{l}\text { Quercus pubescens Willd. } \\
\text { BETULACEAE }\end{array}$ & $\mathrm{P}$ & Orof.SE-Eur. & L-543 & - & & & G1.7 & & & \\
\hline 398. & $\begin{array}{l}\text { Betula pendula Roth. } \\
\text { LYTHRACEAE }\end{array}$ & $\mathrm{P}$ & Euro-Siber. & L-151 & - & & & G1.7, G4.6, E5.2 & & & \\
\hline 399. & $\begin{array}{l}\text { Lythrum salicaria } \mathrm{L} . \\
\text { ONAGRACEAE }\end{array}$ & $\mathrm{H}$ & Subcosmop. & L-544 & - & & & D4.1, D2.3, D5.131 & LC & & \\
\hline 400. & Circaea lutetiana $\mathrm{L}$. & $\mathrm{H}$ & Circumbor. & L-190 & - & & & $\begin{array}{l}\text { G1.1, E5.43, E5.2, } \\
\text { G1.A1 }\end{array}$ & & & \\
\hline 401. & Epilobium angustifolium L. & $\mathrm{H}$ & Circumbor. & L-191 & & & & $F 2.231, F 2.2, E 1.92$ & & & \\
\hline 402. & Epilobium montanum $\mathrm{L}$. & $\mathrm{H}$ & Euro-Asiat. & L-192 & - & & & $\begin{array}{l}\text { G1.6933, E4.3, E5.2, } \\
\text { D2.38 }\end{array}$ & & & \\
\hline & SAPINDACEAE & & & & & & & & & & \\
\hline 403. & Acer campestre L. & $\mathrm{P}$ & Euro-Cauc. & L-197 & $\bullet$ & & & $\begin{array}{l}\text { F2.2, F2.23, F2.263, } \\
\text { E5.2, G1.A1 }\end{array}$ & & & \\
\hline 404. & Acer heldreichii Orph. ex Boiss. & $\mathrm{P}$ & Balkan & L-198 & & & EN & G1.6933 & & End(B) & $\boldsymbol{\nabla}$ \\
\hline 405. & Acer hyrcanum subsp. intermedium (Pančić) Bornm. & $\mathrm{P}$ & Balkan & N/A & - & & & F3.2421 & & End(B) & \\
\hline 406. & Acer obtusatum Willd. & $\mathrm{P}$ & Euri-Medit. & L-545 & • & & & F3.242, G1.6924 & & & \\
\hline 407. & Acer platanoides $\mathrm{L}$. & $\mathrm{P}$ & Euro-Asiat. & L-199 & $\bullet$ & & & $\begin{array}{l}\text { F3.2421, G1.6933, E5.2, } \\
\text { G1.A1 }\end{array}$ & & & \\
\hline 408. & Acer pseudoplatanus $\mathrm{L}$. & $\mathrm{P}$ & Europ. & L-200 & - & & & $\begin{array}{l}\text { F2.2, F2.23, G1.A1, } \\
\text { F3.2421, G3.1 }\end{array}$ & & & \\
\hline
\end{tabular}


Annex. 1 - cont.

\begin{tabular}{|c|c|c|c|c|c|c|c|c|c|c|c|}
\hline & FAMILY / Taxon & Life & Flor. element & Herb. & R.84 & R.90 & XK & EUNIS code & EU & END & Note \\
\hline 409. & $\begin{array}{l}\text { Acer tataricum } \mathrm{L} \text {. } \\
\text { THYMELACEAE }\end{array}$ & $\mathrm{P}$ & Euro-Asiat. & L-546 & $\bullet$ & & & G4.6, E5.43, G1.A1 & & & \\
\hline 410. & Daphne laureola L. & $\mathrm{P}$ & Euri-Medit. & L-547 & - & & & G1.6933 & & & \\
\hline 411. & Daphne mezereum L. & $\mathrm{P}$ & Euro-Siber. & L-149 & $\bullet$ & - & & $\begin{array}{l}\text { F2.2, E4.41, E4.412, } \\
\text { G3.1 }\end{array}$ & & & \\
\hline 412. & $\begin{array}{l}\text { Daphne oleoides Schreb. } \\
\text { CORYLACEAE }\end{array}$ & c & Medit.-Mont. & L-148 & & & & $\mathrm{H} 2.6, \mathrm{H} 2.4, \mathrm{E} 4.4$ & & & \\
\hline 413. & Carpinus betulus L. & $\mathrm{P}$ & Euro-Cauc. & L-548 & - & & & F3.2421, G1.A1 & & & \\
\hline 414. & Carpinus orientalis Mill. & $\mathrm{P}$ & Pontic & L-549 & - & & & F3.2421, G1.7, G1.A1 & & & \\
\hline 415. & Corylus avellana L. & $\mathrm{P}$ & Euro-Cauc. & L-550 & - & & & G1.7 & & & \\
\hline 416. & Corylus colurna $\mathrm{L}$. & $\mathrm{P}$ & Euro-Asiat. & L-551 & - & & & G1.6924 & & & \\
\hline 417. & $\begin{array}{l}\text { Ostrya carpinifolia Scop. } \\
\text { CISTACEAE }\end{array}$ & $\mathrm{P}$ & Circumbor. & L-153 & - & & & G1.6924, G1.7C1 & & & \\
\hline 418. & Helianthemum alpestre (Jacq.) DC. & c & Euri-Medit. & L-705 & & - & & E4.4, E4.41 & & & \\
\hline 419. & Helianthemum canum (L.) Baumg. & c & Euro-Cauc. & L-181 & $\bullet$ & - & & $\begin{array}{l}\text { E1.22, E4.4122, } \\
\text { E4.4126, H2.4 }\end{array}$ & & & \\
\hline 420. & Helianthemum nummularium (L.) Mill. subsp. nummularium & c & Euro-Cauc. & L-182 & $\bullet$ & $\bullet$ & & $\begin{array}{l}\text { E1.2, E1.22, E1.73, } \\
\text { E1.92 }\end{array}$ & & & \\
\hline 421. & $\begin{array}{l}\text { Helianthemum nummularium subsp. grandiflorum (Scop.) } \\
\text { Schinz \& Thell. }\end{array}$ & c & Orof.S.Eur. & L-183 & & & & E3.4, E1.2, E4.3 & & & \\
\hline 422. & $\begin{array}{l}\text { MALVACEAE } \\
\text { Alcea biennis Winterl }\end{array}$ & $\mathrm{H}$ & Medit.-Mont. & N/A & - & & & I1.3 & & & \\
\hline 423. & Malva sylvestris L. & $\mathrm{T}$ & Euro-Asiat. & L-552 & - & & & 11.3 & & & \\
\hline 424. & Tilia platyphyllos Scop. & $\mathrm{P}$ & Euro-Asiat. & L-553 & - & & & G1.7 & & & \\
\hline 425. & $\begin{array}{l}\text { Tilia tomentosa Moench } \\
\text { RESEDACEAE }\end{array}$ & $\mathrm{P}$ & Euro-Asiat. & L-139 & $\bullet$ & & & G1.7, E5.2, G1.A1 & & & \\
\hline 426. & $\begin{array}{l}\text { Reseda lutea } \mathrm{L} . \\
\text { BRASSICACEAE }\end{array}$ & $\mathrm{H}$ & Europ. & L-554 & - & & & E1.2 & & & \\
\hline 427. & Aethionema saxatile (L.) W. T. Aiton & c & Medit.-Mont. & L-161 & - & & & E1.2, E1.22, E1.7 & & & \\
\hline 428. & Alyssum montanum L. & c & Centro-Eur. & L-162 & - & & & E1.2, E4.4, E4.41 & & & \\
\hline 429. & Alyssum scardicum Wettst. & C & Balkan & L-555 & & - & & E4.4, E4.41, E4.412 & & End(B) & \\
\hline 430. & Arabis alpina subsp. caucasica (Willd.) Briq. & $\mathrm{H}$ & Medit.-Mont. & L-164 & - & $\bullet$ & & E4.1, E4.5, H2.4 & & & \\
\hline 431. & Arabis ciliata Clairv. & H & Orof.SE.Eur. & L-165 & & & & E4.4, E4.41, E1.73 & & & $\boldsymbol{\nabla}$ \\
\hline 432. & Arabis hirsuta (L.) Scop. & $\mathrm{H}$ & Europ. & L-166 & & & & E1.2, F3.2421 & & & $\boldsymbol{\nabla}$ \\
\hline 433. & Arabis serpillifolia Vill. & $\mathrm{H}$ & Medit.-Mont. & L-188 & & & & $\mathrm{H} 2.4$ & & & $\boldsymbol{\nabla}$ \\
\hline 434. & $\begin{array}{l}\text { Aubrieta columnae subsp. croatica (Schott, Nyman, } \\
\text { Kotschy)Mattf. }\end{array}$ & c & Balkan & L-167 & $\bullet$ & & CR & $\begin{array}{l}\mathrm{H} 3.216, \mathrm{H} 3.2 \mathrm{~A} 13, \mathrm{H} 2.4 \\
\text { E1.112 }\end{array}$ & & & \\
\hline
\end{tabular}


Annex. 1 - cont.

\begin{tabular}{|c|c|c|c|c|c|c|c|c|c|c|c|}
\hline & FAMILY / Taxon & Life & Flor. element & Herb. & R.84 & R.90 & $\mathrm{XK}$ & EUNIS code & EU & END & Note \\
\hline 435. & Barbarea balcana Pančić & $\mathrm{H}$ & Balkan & L-180 & & & NT & E5.57 & LC & End(B) & \\
\hline 436. & Barbarea vulgaris $\mathrm{R} . \mathrm{Br}$. & $\mathrm{H}$ & Cosmopol. & L-168 & & & & G1.7, D2.26 & LC & & \\
\hline 437. & Capsella bursa-pastoris (L.) Medik. & $\mathrm{H}$ & Cosmopol. & L-556 & $\bullet$ & & & G1.7, E5.4 & & & \\
\hline 438. & Cardamine bulbifera (L.) Crantz. & G & Centro-Eur. & L-169 & $\bullet$ & & & G1.6933, G1.A1 & & & \\
\hline 439. & Cardamine carnosa Waldst. et Kit. & $\mathrm{H}$ & Balkan & L-170 & & & & $\begin{array}{l}\text { H3.2A13, D2.38, D2.26, } \\
\text { H2.3 }\end{array}$ & & End(B) & $\nabla$ \\
\hline 440. & Cardamine enneaphyllos (L.) Crantz & G & Orof.SE-Eur. & L-171 & $\bullet$ & & & G1.6933 & & & \\
\hline 441. & Cardamine flexuosa With. & $\mathrm{H}$ & Circumbor. & L-172 & & & & G1.6933, E5.4, E5.2 & & & \\
\hline 442. & Cardamine glauca DC. subsp. glauca & $\mathrm{T}$ & Balkan & L-707 & & & & $\mathrm{D} 4.1, \mathrm{H} 2.4$ & & & \\
\hline 443. & Cardamine hirsuta $\mathrm{L}$. & $\mathrm{T}$ & Medit.-Mont. & L-706 & & & & E5.4 & & & \\
\hline 444. & Cardamine impatiens L. & $\mathrm{T}$ & Euro-Asiat. & L-708 & & & & $\mathrm{E} 5.4, \mathrm{G} 1.7$ & & & \\
\hline 445. & Cardamine pratensis L. & $\mathrm{H}$ & Europ. & L-174 & $\bullet$ & & & D2.2, G1.69322 & LC & & \\
\hline 446. & Draba aizoides $\mathrm{L}$. & $\mathrm{H}$ & Orof.C.Eur. & L-695 & & $\bullet$ & & E4.4, E4.41, E4.4126 & & & \\
\hline 447. & Draba doerfleri Wettst. & C & Balkan & L-557 & & & & H3.152 & & End(B) & \\
\hline 448. & Draba praecox Steven & $\mathrm{T}$ & Steno-Medit. & L-655 & & $\bullet$ & & $\mathrm{E} 4.392, \mathrm{E} 1.7$ & & & \\
\hline 449. & Draba scardica (Griseb.) Degen \& Dörfler & $\mathrm{H}$ & Balkan & L-175 & $\bullet$ & & & $\mathrm{E} 4.41, \mathrm{H} 3.2, \mathrm{H} 2.4$ & & & \\
\hline 450. & Draba verna $\mathrm{L}$. & $\mathrm{T}$ & Euro-Asiat. & L-176 & $\bullet$ & & & E3.4, E3.5 & & & \\
\hline 451. & Erysimum carniolicum Dolliner & $\mathrm{H}$ & Balkan & N/A & $\bullet$ & & & E1.2 & & & \\
\hline 452. & Erysimum comatum Pančić & $\mathrm{H}$ & Balkan & L-154 & & & & E4.41, E4.412, E4.4126 & & & $\nabla$ \\
\hline 453. & Erysimum diffusum Ehrh. & $\mathrm{H}$ & Europ. & N/A & $\bullet$ & & & $\mathrm{E} 1.2, \mathrm{H} 2.6$ & & & \\
\hline 454. & Iberis sempervirens $\mathrm{L}$. & $\mathrm{C}$ & Medit. & L-155 & & $\bullet$ & LC & $\mathrm{E} 4.4, \mathrm{E} 4.41, \mathrm{E} 4.4125$ & & & \\
\hline 455. & Lepidium draba L. & G & Cosmopol. & L-654 & $\bullet$ & & & G1.7 & & & \\
\hline 456. & Noccaea bellidifolia (Griseb.) F. K. Mey. & $\mathrm{H}$ & Balkan & L-156 & $\bullet$ & $\bullet$ & & $\begin{array}{l}\text { E4.41, E4.412, H2.6, } \\
\text { E4.34 }\end{array}$ & & End(B) & \\
\hline 457. & Noccaea goesingensis (Halácsy) F. K. Mey. & $\mathrm{H}$ & Euro-Asiat. & N/A & $\bullet$ & & & G4.6 & & & \\
\hline 458. & Noccaea ochroleuca (Boiss. \& Heldr.) F. K. Mey. & $\mathrm{H}$ & Balkan & L-558 & & & & $\begin{array}{l}\text { G1.6933, E1.7, E1.73, } \\
\text { E4.31 }\end{array}$ & & & \\
\hline 459. & Noccaea perfoliata (L.) Al-Shehbaz & $\mathrm{T}$ & Paleotemp. & N/A & $\bullet$ & & & F3.2421 & & & \\
\hline 460. & Noccaea praecox (Wulfen) F. K. Mey. & $\mathrm{H}$ & Orof.SE.Eur. & L-157 & & & & $\mathrm{E} 1.2, \mathrm{E} 4.31$ & & & \\
\hline 461. & Odontarrhena markgrafii (O. E. Schulz) Španiel \& al. & C & Balkan & N/A & $\bullet$ & & & E1.2, F3.242 & & & \\
\hline 462. & Odontarrhena muralis (Waldst. \& Kit.) Endl. & $\mathrm{H}$ & Euro-Asiat. & N/A & $\bullet$ & & & E1.2 & & & \\
\hline 463. & Phyllolepidum rupestre (Ten.) Trinajstić & $\mathrm{C}$ & Orof.SE.Eur. & L-158 & & $\bullet$ & EN & E4.41731 & & & \\
\hline 464. & Pseudoturritis turrita (L.) Al-Shehbaz & $\mathrm{H}$ & Euro-Asiat. & L-653 & $\bullet$ & & & F3.242 & & & \\
\hline 465. & Rorippa pyrenaica (All.) Rchb. & $\mathrm{H}$ & Europ. & N/A & $\bullet$ & & & E5.3 & LC & & \\
\hline 466. & Rorippa sylvestris (L.) Besser & $\mathrm{H}$ & Europ. & L-559 & $\bullet$ & & & D5.131 & LC & & \\
\hline 467. & Thlaspi arvense L. & $\mathrm{T}$ & Cosmopol. & L-560 & $\bullet$ & & & E5.4 & & & \\
\hline 468. & Turritis glabra L. & $\mathrm{H}$ & Europ. & L-159 & & & & $\mathrm{E} 1.2, \mathrm{E} 1.22, \mathrm{G} 1.7$ & & & \\
\hline
\end{tabular}


Annex. 1 - cont.

\section{SANTALACEAE}

469. Thesium alpinum $\mathrm{L}$.

470. Thesium linophyllon subsp. montanum (Schrad.) Čelak.

471. Thesium parnassi A. DC.

\section{PLUMBAGINACEAE}

472. Armeria canescens (Host) Boiss. POLYGONACEAE

473. Aconogonon alpinum (All.) Schur

474. Bistorta officinalis Delarbre

475. Bistorta vivipara (L.) Delarbre

476. Polygonum aviculare L.

477. Rumex acetosa L.

478. Rumex acetosella L.

479. Rumex alpinus L.

480. Rumex crispus $\mathrm{L}$.

\section{AMARANTHACEAE}

481. Amaranthus retroflexus $\mathrm{L}$.

\section{CARYOPHYLLACEAE}

$\mapsto \quad 482$. Arenaria rotundifolia M. Bieb.

483. Arenaria serpyllifolia $\mathrm{L}$

484. Cerastium alpinum $\mathrm{L}$.

485. Cerastium brachypetalum Pers

486. Cerastium cerastoides (L.) Britton.

487. Cerastium decalvans Schloss. \& Vuk.

488. Cerastium fontanum subsp. vulgare (Hartm.) Greut. \& Burd.

489. Cerastium glomeratum Thuill.

490. Dianthus armeria $\mathrm{L}$.

491. Dianthus carthusianorum L.

492. Dianthus cruentus Griseb.

493. Dianthus deltoides subsp. degenii (Bald.) Strid.

494. Dianthus gracilis subsp. armerioides (Griseb.) Tutin

495. Dianthus integer subsp. minutiflorus (Halácsy) Bornm.

496. Dianthus scardicus Wettst.

\begin{tabular}{|c|c|c|c|c|c|c|}
\hline Life & Flor. element & Herb. & R. 84 & R.90 & XK & EUNIS code \\
\hline $\mathrm{H}$ & Art.Alp. & L-140 & & $\bullet$ & & E4.3, E4.392 \\
\hline $\mathrm{H}$ & Europ. & N/A & & $\bullet$ & & E1.2 \\
\hline $\mathrm{H}$ & Orof.S.Eur. & N/A & & $\bullet$ & & E4.4125 \\
\hline $\mathrm{H}$ & Orof.SE.Eur. & L-562 & $\bullet$ & & NT & E4.41, E4.412, E4.4125 \\
\hline $\mathrm{H}$ & Paleotemp. & N/A & & $\bullet$ & & E1.74 \\
\hline G & Circumbor. & L-563 & & $\bullet$ & & $\mathrm{D} 2.2$ \\
\hline G & Art.Alp. & L-336 & $\bullet$ & & & $\mathrm{F} 2.2, \mathrm{E} 4.34$ \\
\hline $\mathrm{T}$ & Cosmopol. & N/A & $\bullet$ & & & E5.4 \\
\hline $\mathrm{H}$ & Circumbor. & L-338 & & $\bullet$ & & $\mathrm{E} 1.2, \mathrm{E} 1.22$ \\
\hline $\mathrm{H}$ & Subcosmop. & L-339 & $\bullet$ & & & E1.2, E1.7, E4.34 \\
\hline $\mathrm{H}$ & Euro-Cauc. & L-337 & $\bullet$ & $\bullet$ & & E5.58, F2.3 \\
\hline $\mathrm{H}$ & Subcosmop. & L-340 & $\bullet$ & & & E5.4, E5.2, E3.3 \\
\hline $\mathrm{T}$ & N. Amer. & L-652 & $\bullet$ & & & I1.3, E5.4 \\
\hline C & Art.Alp. & $\mathrm{N} / \mathrm{A}$ & $\bullet$ & & & H3.15, H3.152 \\
\hline $\mathrm{T}$ & Subcosmop. & L-651 & $\bullet$ & $\bullet$ & & E1.12, E4.4 \\
\hline H & Art.Alp. & L-184 & $\bullet$ & & & $\begin{array}{l}\text { E4.4, E4.42, E4.412, } \\
\text { H3.152 }\end{array}$ \\
\hline $\mathrm{T}$ & Euri-Medit. & N/A & $\bullet$ & & & $\mathrm{E} 1.2, \mathrm{E} 4.31$ \\
\hline $\mathrm{H}$ & Art.Alp. & L-186 & $\bullet$ & & & $\begin{array}{l}\text { D2.2, E4.1, E4.3, E4.34, } \\
\text { E4.5 }\end{array}$ \\
\hline C & Orof.SE.Eur. & L-187 & & $\bullet$ & & $\mathrm{H} 2.6, \mathrm{H} 3.2, \mathrm{H} 2.4$ \\
\hline $\mathrm{H}$ & Euro-Asiat. & N/A & & $\bullet$ & & E1.2 \\
\hline $\mathrm{T}$ & Euri-Medit. & N/A & $\bullet$ & & & E1.92 \\
\hline $\mathrm{H}$ & Euro-Siber. & L-189 & $\bullet$ & & & $\mathrm{E} 1.2, \mathrm{E} 1.22, \mathrm{G} 1.7$ \\
\hline $\mathrm{H}$ & Centro-Eur. & L-696 & $\bullet$ & & & $\mathrm{E} 1.2, \mathrm{E} 1.22, \mathrm{E} 4.3$ \\
\hline $\mathrm{H}$ & Balkan & L-241 & & $\bullet$ & & E1.2, E1.7, E1.73 \\
\hline $\mathrm{H}$ & Balkan & L-242 & & & & E1.7, E1.73, F2.231 \\
\hline $\mathrm{H}$ & Balkan & L-342 & & & & E1.7 \\
\hline $\mathrm{H}$ & Balkan & L-243 & & & & $\begin{array}{l}\text { E4.4, E4.41, E4.412, } \\
\text { H2.4 }\end{array}$ \\
\hline $\mathrm{H}$ & Balkan & L-245 & $\bullet$ & $\bullet$ & NT & $\begin{array}{l}\text { E4.3, E4.31, E4.312, } \\
\text { E1.112 }\end{array}$ \\
\hline
\end{tabular}

$\operatorname{End}(B)$ 
Annex. 1 - cont.

\begin{tabular}{|c|c|c|c|c|c|c|c|c|c|c|c|}
\hline & FAMILY / Taxon & Life & Flor. element & Herb. & R.84 & R.90 & XK & EUNIS code & EU & END & Note \\
\hline 497. & Dianthus superbus L. & $\mathrm{H}$ & Euro-Asiat. & L-566 & & $\bullet$ & LC & E3.4, E3.5, E4.4126 & & & \\
\hline 498. & Dianthus sylvestris subsp. bertisceus Rech. f. & $\mathrm{H}$ & Balkan & L-246 & & & & $\begin{array}{l}\text { E4.5, E4.4122, H2.4, } \\
\text { E1.112 }\end{array}$ & & End(B) & \\
\hline 499. & Dianthus sylvestris Wulfen subsp. sylvestris & $\mathrm{H}$ & Medit.-Mont. & L-247 & $\bullet$ & $\bullet$ & & E4.5, E4.41 & & & \\
\hline 500. & Drypis spinosa L. & $\mathrm{H}$ & Balkan & L-248 & $\bullet$ & & EN & $\mathrm{H} 2.3$ & & & \\
\hline 501. & Herniaria glabra L. & $\mathrm{T}$ & Paleotemp. & N/A & $\bullet$ & & & E1.92, G1.7 & & & \\
\hline 502. & Minuartia graminifolia subsp. clandestina (Port.) Mattf. & $\mathrm{C}$ & Medit.-Mont. & $\mathrm{L}-250$ & & & & $\mathrm{E} 1.92, \mathrm{H} 3.15$ & & End(B) & \\
\hline 503. & Minuartia recurva (All.) Schinz \& Thell. & C & Euro-Cauc. & L-216 & $\bullet$ & & & $\begin{array}{l}\text { E4.3, E4.39, E4.3925, } \\
\text { E4.34 }\end{array}$ & & & \\
\hline 504. & Minuartia verna (L.) Hiern. & $\mathrm{C}$ & Euro-Asiat. & L-217 & $\bullet$ & $\bullet$ & & $\begin{array}{l}\text { E1.2, E1.22, E4.4, E4.41, } \\
\mathrm{H} 2.4\end{array}$ & & & \\
\hline 505. & Moenchia mantica (L.) Bartl. & $\mathrm{T}$ & Medit.-Mont. & L-218 & $\bullet$ & & & $\mathrm{E} 1.2, \mathrm{E} 1.22$ & & & \\
\hline 506. & Paronychia kapela (Hacq.) A.Kern & $\mathrm{H}$ & Medit.-Mont. & L-219 & & & & E1.2, E4.4, E4.41 & & & \\
\hline 507. & Paronychia macedonica Chaudhri & $\mathrm{H}$ & Balkan & $\mathrm{L}-721$ & & & & E4.41, E4.4126 & & & \\
\hline 508. & Petrorhagia prolifera (L.) P. W. Ball \& Heywood & $\mathrm{T}$ & Euri-Medit. & $\mathrm{N} / \mathrm{A}$ & $\bullet$ & & & G1.7 & & & \\
\hline 509. & Petrorhagia saxifraga (L.) Link & $\mathrm{H}$ & Euri-Medit. & L-650 & $\bullet$ & & & $\mathrm{E} 1.2, \mathrm{H} 2.6, \mathrm{E} 1.92$ & & & \\
\hline 510. & Sagina subulata (Sw.) C. Presl & $\mathrm{H}$ & Submedit. & L-220 & & & & D4.1 & & & \\
\hline 511. & Saponaria officinalis L. & $\mathrm{H}$ & Euro-Siber. & L-720 & & & & E5.4 & & & \\
\hline 512. & Scleranthus perennis subsp. marginatus (Guss.) Nyman & $\mathrm{H}$ & Orof.S.Eur. & L-264 & $\bullet$ & $\bullet$ & & $\begin{array}{l}\text { E1.22, E4.3, E4.31, } \\
\text { E4.34 }\end{array}$ & & & \\
\hline 513. & Silene asterias Griseb. & G & Balkan & L-567 & $\bullet$ & & NT & $\mathrm{D} 2.2, \mathrm{D} 2.26, \mathrm{D} 2.282$ & & End(B) & \\
\hline 514. & Silene bupleuroides subsp. staticifolia (Sm.) Chowdhuri & $\mathrm{H}$ & Euro-Asiat. & L-265 & & & & E1.2 & & & \\
\hline 515. & Silene ciliata Pourr. & $\mathrm{H}$ & Medit.-Mont. & L-266 & & $\bullet$ & & E1.112, E1.92 & & & \\
\hline 516. & Silene coronaria (L.) Clairv. & $\mathrm{H}$ & Medit.-Mont. & L-568 & $\bullet$ & & & F3.242, G1.7 & & & \\
\hline 517. & Silene dioica (L.) Claivr. & $\mathrm{H}$ & Paleotemp. & L-267 & & & & E5.5721 & & & \\
\hline 518. & Silene flos-cuculi (L.) Clairv. & $\mathrm{H}$ & Euro-Siber. & $\mathrm{N} / \mathrm{A}$ & $\bullet$ & & & G1.7, E3.3 & & & \\
\hline 519. & Silene italica (L.) Pers. & $\mathrm{H}$ & Euri-Medit. & L-569 & $\bullet$ & & & G1.7 & & & \\
\hline 520. & Silene parnassica Boiss. \& Spruner subsp. parnassica & $\mathrm{H}$ & Balkan & L-268 & & & & E4.5 & & End(B) & \\
\hline 521. & Silene pusilla Waldst. \& Kit. & $\mathrm{H}$ & Orof.S.Eur. & L-269 & $\bullet$ & & & $\begin{array}{l}\text { D4.1, E4.41, D2.3, } \\
\text { D2.38 }\end{array}$ & & & \\
\hline 522. & Silene saxifraga $\mathrm{L}$. & $\mathrm{H}$ & Orof.S.Eur. & $\mathrm{L}-270$ & $\bullet$ & $\bullet$ & & $\begin{array}{l}\text { E4.4, E4.41, E4.412, } \\
\text { H2.4 }\end{array}$ & & & \\
\hline 523. & Silene sendtneri Boiss. & $\mathrm{H}$ & Balkan & L-271 & $\bullet$ & $\bullet$ & & $\begin{array}{l}\text { E1.22, E1.7, E4.31, } \\
\text { E4.39, E4.34 }\end{array}$ & & End(B) & \\
\hline 524. & Silene viridiflora $\mathrm{L}$. & $\mathrm{H}$ & Orof.S.Eur. & L-570 & $\bullet$ & & & F3.2421, G1.7, G1.A1 & & & \\
\hline 525. & Silene viscaria (L.) Jess. & $\mathrm{H}$ & Euro-Siber. & L-272 & $\bullet$ & $\bullet$ & & E1.73 & & & \\
\hline 526. & Silene vulgaris (Moench) Garcke subsp. vulgaris & $\mathrm{H}$ & Subcosmop. & L-273 & $\bullet$ & & & E1.22, E1.73 & & & \\
\hline
\end{tabular}


Annex. 1 - cont.

\begin{tabular}{|c|c|c|c|c|c|c|c|c|c|c|c|}
\hline & FAMILY / Taxon & Life & Flor. element & Herb. & R.84 & R.90 & $\mathrm{XK}$ & EUNIS code & EU & END & Note \\
\hline 527. & Silene vulgaris subsp. prostrata (Gaudin) Schinz \& Thell & $\mathrm{H}$ & Orof.SW.Eur. & L-274 & & • & & E4.31 & & & \\
\hline 528. & Silene waldsteinii Griseb. & $\mathrm{H}$ & Balkan & L-275 & & • & & H2.6, H3.15 & & $\operatorname{End}(B)$ & \\
\hline 529. & Spergularia rubra (L.) J. Presl \& C. Presl & $\mathrm{T}$ & Paleotemp. & L-276 & & & & E5.4, E5.43 & & & \\
\hline 530. & Stellaria alsine Grimm & $\mathrm{H}$ & Euro-Siber. & L-277 & & & & $\begin{array}{l}\text { D2.2, D2.26, D2.38, } \\
\text { D2.26 }\end{array}$ & & & \\
\hline 531. & Stellaria graminea $\mathrm{L}$. & $\mathrm{H}$ & Euro-Siber. & L-278 & & • & & E1.2, E1.7, E4.39, D2.38 & & & \\
\hline 532. & Stellaria holostea L. & C & Euro-Cauc. & L-571 & $\bullet$ & $\bullet$ & & $\begin{array}{l}\text { F3.2421, G1.6933, } \\
\text { E4.392, G1.A1 }\end{array}$ & & & \\
\hline 533. & Stellaria media (L.) Cirillo & $\mathrm{T}$ & Cosmopol. & L-712 & & & & $\mathrm{E} 5.58, \mathrm{G} 1.7$ & & & \\
\hline 534. & $\begin{array}{l}\text { Stellaria nemorum L. subsp. nemorum } \\
\text { CHENOPODIACEAE }\end{array}$ & $\mathrm{H}$ & Europ. & L-280 & & & & $\mathrm{D} 2.28$ & & & \\
\hline 535. & Atriplex patula $\mathrm{L}$. & $\mathrm{T}$ & Euro-Siber. & L-649 & • & & & E5.4 & & & \\
\hline 536. & $\begin{array}{l}\text { Blitum bonus-henricus (L.) Rchb. } \\
\text { CORNACEAE }\end{array}$ & $\mathrm{H}$ & Europ. & L-697 & - & & & E5.4 & & & \\
\hline 537. & Cornus mas L. & $\mathrm{P}$ & Euro-Asiat. & L-572 & - & & & F3.2421, G1.A1 & & & \\
\hline 538. & $\begin{array}{l}\text { Cornus sanguinea } \mathrm{L} . \\
\text { PRIMULACEAE }\end{array}$ & $\mathrm{P}$ & Euro-Siber. & L-573 & $\bullet$ & & & F3.2421, G1.1, G1.A1 & & & \\
\hline 539. & Anagallis arvensis $\mathrm{L}$. & $\mathrm{T}$ & Cosmopol. & L-698 & • & & & E5.43 & & & \\
\hline 540. & Anagallis foemina Mill. & $\mathrm{T}$ & Euro-Asiat. & N/A & • & & & E5.43 & & & \\
\hline 541. & Cyclamen hederifolium Aiton & G & Euri-Medit. & L-574 & • & & & G1.7 & & & \\
\hline 542. & Lysimachia nummularia L. & H & Euro-Asiat. & L-648 & $\bullet$ & & & G1.7, G1.A1 & & & \\
\hline 543. & Lysimachia punctata L. & $\mathrm{H}$ & Europ. & L-347 & & & & $\begin{array}{l}\text { G1.69322, G1.6933, } \\
\text { E5.2 }\end{array}$ & & & \\
\hline 544. & Lysimachia vulgaris $\mathrm{L}$. & $\mathrm{H}$ & Euro-Asiat. & L-575 & - & & & D2.2, D2.22 & LC & & \\
\hline 545. & Primula acaulis (L.) L. & $\mathrm{H}$ & Euro-Asiat. & L-348 & - & & & $\begin{array}{l}\text { G1.6924, G1.7, E5.2, } \\
\text { G1.A1 }\end{array}$ & & & \\
\hline 546. & Primula elatior subsp. intricata (Gren. \& Godr.) Widmer & $\mathrm{H}$ & Orof.S.Eur. & N/A & & • & & E4.392 & & & \\
\hline 547. & Primula minima $\mathrm{L}$. & $\mathrm{H}$ & Orof.SE.Eur. & L-350 & • & • & & F2.2, E4.3, E4.39 & & & \\
\hline 548. & Primula veris subsp. columnae (Ten.) Lüdi & $\mathrm{H}$ & Europ. & L-352 & • & & & F3.242 & & & \\
\hline 549. & $\begin{array}{l}\text { Soldanella alpina } \mathrm{L} . \\
\text { ERICACEAE }\end{array}$ & $\mathrm{H}$ & Orof.S.Eur. & L-353 & & $\bullet$ & & E4.3, E4.31, E4.34 & & & \\
\hline 550. & Arctostaphylos uva-ursi (L.) Spreng & C & Art.Alp. & L-375 & $\bullet$ & $\bullet$ & & $\begin{array}{l}\text { E4.4, E4.41, E4.4122, } \\
\text { E4.34 }\end{array}$ & LC & & \\
\hline 551. & Bruckenthalia spiculifolia Griseb. & C & Balkan & L-376 & $\bullet$ & $\bullet$ & & $\begin{array}{l}\text { E1.7, E1.73, E3.5, E4.3, } \\
\text { E4.34 }\end{array}$ & & & \\
\hline 552. & Empetrum hermaphroditum Hagerup & c & Circumbor. & L-377 & & - & & $\mathrm{E} 4.3, \mathrm{E} 4.39$ & & & \\
\hline 553. & Rhododendron ferrugineum L. & $\mathrm{P}$ & Orof.S.Eur. & L-378 & & - & LC & $F 2.2, F 2.22$ & & & \\
\hline
\end{tabular}


Annex. 1 - cont.

\begin{tabular}{|c|c|c|c|c|c|c|c|c|c|c|c|}
\hline & FAMILY / Taxon & Life & Flor. element & Herb. & R.84 & R.90 & XK & EUNIS code & EU & END & Note \\
\hline 554. & Vaccinium myrtillus L. & C & Circumbor. & L-379 & $\bullet$ & $\bullet$ & & $\begin{array}{l}\text { E1.71, F2.22, E4.34, } \\
\text { G3.1, H2.3 }\end{array}$ & & & \\
\hline 555. & $\begin{array}{l}\text { Vaccinium uliginosum } \mathrm{L} \text {. } \\
\text { RUBIACEAE }\end{array}$ & $\mathrm{C}$ & Circumbor. & L-380 & $\bullet$ & $\bullet$ & & $\mathrm{F} 2.2, \mathrm{E} 4.3, \mathrm{H} 2.3$ & & & \\
\hline 556. & Asperula aristata L. f. & $\mathrm{H}$ & Euri-Medit. & L-647 & & $\bullet$ & VU & F2.231, G1.7C1 & & & \\
\hline 557. & Asperula arvensis L. & $\mathrm{T}$ & Euri-Medit. & L-576 & $\bullet$ & & & E5.4 & & & \\
\hline 558. & Asperula cynanchica L. & $\mathrm{H}$ & Euri-Medit. & L-364 & $\bullet$ & & & $\begin{array}{l}\text { E4.4126, E4.41731, } \\
\text { F2.231, H2.4 }\end{array}$ & & & \\
\hline 559. & Asperula doerfleri Wettst. & $\mathrm{C}$ & Balkan & $\mathrm{L}-470$ & $\bullet$ & & LC & $\mathrm{E} 4.4, \mathrm{H} 3.2 \mathrm{~A} 13$ & & End(B) & \\
\hline 560. & Asperula longiflora Waldst. \& Kit. & $\mathrm{H}$ & Euri-Medit. & L-577 & & $\bullet$ & & $\mathrm{E} 1.2, \mathrm{E} 4.4$ & & & \\
\hline 561. & Asperula taurina $\mathrm{L}$. & $\mathrm{H}$ & Euro-Asiat. & N/A & $\bullet$ & & & G1.6933, G1.A1 & & & \\
\hline 562. & Cruciata laevipes Opiz & $\mathrm{H}$ & Euro-Asiat. & N/A & $\bullet$ & & & E1.2 & & & \\
\hline 563. & Galium album Mill. subsp. album & $\mathrm{H}$ & Euro-Asiat. & L-578 & & $\bullet$ & & E4.4, E4.41 & & & \\
\hline 564. & Galium anisophyllon Vill. & $\mathrm{H}$ & Euri-Medit. & L-365 & $\bullet$ & $\bullet$ & & $\mathrm{E} 4.3, \mathrm{E} 4.39, \mathrm{H} 2.4$ & & & \\
\hline 565. & Galium aparine L. & $\mathrm{T}$ & Euro-Asiat. & $\mathrm{N} / \mathrm{A}$ & $\bullet$ & & & $\begin{array}{l}\text { E5.43, G1.6933, E5.2, } \\
\text { G1.A1 }\end{array}$ & & & \\
\hline 566. & Galium corrudifolium Vill. & $\mathrm{H}$ & Steno-Medit. & L-366 & $\bullet$ & & & $\mathrm{G} 1.7, \mathrm{H} 2.6, \mathrm{H} 2.6 \mathrm{~B}$ & & & \\
\hline 567. & Galium flavescens Borbás ex Simonk & $\mathrm{H}$ & Balkan & L-367 & & & & E4.413, E4.413A & & & \\
\hline 568. & Galium debile Desv. & $\mathrm{H}$ & Euri-Medit. & N/A & $\bullet$ & & & E5.4, E5.43 & & & \\
\hline 569. & Galium mollugo L. & $\mathrm{H}$ & Euri-Medit. & L-368 & $\bullet$ & & & $\mathrm{E} 1.2, \mathrm{~F} 2.32, \mathrm{E} 5.2$ & & & \\
\hline 570. & Galium odoratum (L.) Scop. & G & Euro-Cauc. & L-369 & $\bullet$ & & & $\begin{array}{l}\text { G1.6933, G3.17, E5.3, } \\
\text { E5.4, E5.2 }\end{array}$ & & & \\
\hline 571. & Galium palustre L. & $\mathrm{H}$ & Euro-Cauc. & L-370 & & & & $\mathrm{D} 2.2, \mathrm{D} 2.38, \mathrm{E} 3.3$ & & & \\
\hline 572. & Galium pumilum Murray & $\mathrm{H}$ & Subatlant. & L-371 & & & & $\mathrm{E} 1.2, \mathrm{G} 1.7, \mathrm{E} 5.3$ & & & \\
\hline 573. & $\begin{array}{l}\text { Sherardia arvensis L. } \\
\text { GENTIANACEAE }\end{array}$ & $\mathrm{T}$ & Euro-Asiat. & N/A & $\bullet$ & & & E1.92, D2.26 & & & \\
\hline 574. & Gentiana albanica (Jáv.) A. W. Hill & $\mathrm{H}$ & Balkan & N/A & & $\bullet$ & & $\mathrm{E} 1.7, \mathrm{E} 1.73$ & & End(B) & \\
\hline 575. & Gentiana punctata $\mathrm{L}$. & $\mathrm{H}$ & Orof.C.Eur. & L-196 & & $\bullet$ & $\mathrm{LC}$ & E1.7, E1.71 & & & \\
\hline 576. & Gentiana utriculosa L. & $\mathrm{T}$ & Orof.SE.Eur. & L-177 & & & & $\mathrm{E} 1.2, \mathrm{E} 1.92, \mathrm{E} 4.392$ & & & \\
\hline 577. & Gentiana verna $\mathrm{L}$. & $\mathrm{H}$ & Euro-Asiat. & L-178 & & $\bullet$ & & E4.4, E4.41, E3.5 & & & \\
\hline 578. & Gentianella bulgarica (Velen) Holub. & $\mathrm{T}$ & Balkan & L-179 & & & VU & $\begin{array}{l}\text { E1.7, E1.73, E1.92, E4.3, } \\
\text { E4.34 }\end{array}$ & & & \\
\hline & APOCYNACEAE & & & & & & & & & & \\
\hline 579. & Vinca herbacea Waldst. \& Kit. & $\mathrm{C}$ & Euri-Medit. & $\mathrm{N} / \mathrm{A}$ & $\bullet$ & & & $\mathrm{E} 1.2$ & & & \\
\hline 580. & Vinca major L. & $\mathrm{C}$ & Euri-Medit. & L-579 & $\bullet$ & & & E5.43 & & & \\
\hline 581. & Vincetoxicum fuscatum (Hornem.) Rchb. f. & G & Euro-Asiat. & N/A & $\bullet$ & & & E1.2 & & & \\
\hline 582. & Vincetoxicum hirundinaria Medik. & $\mathrm{H}$ & Euri-Medit. & $\mathrm{N} / \mathrm{A}$ & $\bullet$ & & & G4.6 & & & \\
\hline
\end{tabular}


R.84

83. Fraxinus ornus $\mathrm{L}$.

584. Ligustrum vulgare $\mathrm{L}$.

PLANTAGINACEAE

585. Digitalis ferruginea $\mathrm{L}$.

586. Digitalis grandiflora Mill.

587. Digitalis laevigata Waldst. \& Kit.

588. Digitalis lanata Ehrh.

589. Globularia cordifolia L.

590. Globularia meridionalis (Podp.) O. Schwarz

591. Gratiola officinalis

592. Linaria alpina (L.) Mill.

593. Linaria angustissima (Loisel.) Borbás

594. Linaria pelisseriana (L.) Mill.

595. Linaria peloponnesiaca Boiss. \& Heldr.

596. Linaria vulgaris Mill.

597. Plantago atrata Hoppe

598. Plantago gentianoides Sibth. \& Sm

599. Plantago holosteum Scop.

600. Plantago lanceolata $\mathrm{L}$.

601. Plantago major L.

602. Plantago media L.

603. Plantago reniformis Beck

604. Veronica alpina L.

605. Veronica aphylla L.

606. Veronica arvensis L.

607. Veronica austriaca L.

608. Veronica austriaca subsp. jacquinii (Baumg.) Eb. Fisch.

609. Veronica austriaca subsp. teucrium (L.) D. A. Webb

610. Veronica beccabunga L.

611. Veronica chamaedrys L.

612. Veronica fruticans Jacq.
Orof.S.Eur

Euro-Cauc.

\section{Euri-Medit.}

Orof.SE-Eur.

Balkan

Balkan

Medit.-Mont.

Circumbor.

Orof.S.Eur.

Orof.SE.Eur.

Medit.-Mont.

Balkan

Euro-Asiat.

Europ.

Balkan

Europ.

Cosmopol.

Euro-Asiat.

Euro-Asiat.

Balkan

Euro-Asiat.

Orof.S.Eur.

Euro-Asiat.

Centro-Eur.

Euro-Asiat.

Centro-Eur.

Euro-Asiat.

Euro-Asiat.

Art.Alp.

\section{Medit.-Mont.}

Herb.

L-261

L-262

\section{L-580}

L-381

L-581

N/A

R.90

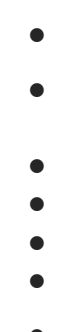

$\bullet$

$\bullet$

$\bullet$

$\bullet$

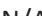

L-582

L-383

L-384

N/A

L-385

L-583

L-471

L-472

L-386

L-387

L-388

L-584

L-473

N/A

L-474

L-585

L-389

L-390

L-391

L-392

L-393

L-394
G1.7, G1.A1, G1.1,

G1.6933

F3.2421, G1.A1

F2.24, F2.231

E5.43, E5.5, F2.231

F3.2421, G1.7

E5.43, G1.7

E4.4, E4.41, F3.2421

E1.22

E4.1, E4.5

D2.22

CR H2.6, H2.6B, H2.3

F3.2421, G1.7

E1.7

E4.392, E4.412

E1.7, F3.242, E4.39

E4.1, E4.116, E4.12,

E4.14

LC E4.1, E4.14, E4.5

E1.92, E4.4, E4.41

$E 1.2, E 5.2, E 3.3$

E3.4, E4.39, E5.2

E1.2, E1.22

NT E5.51, E5.58, E4.4121

E4.1, E4.39, E4.5

E4.412, E4.4125,

E4.3925

E5.43

E1.2, F3.2421, E1.7

E1.22

$\mathrm{E} 1.7, \mathrm{E} 1.73$

E3.4, E3.5, D2.38, D2.26 LC

E1.22, E5.2, E4.34

G3.1, G1.A1

E4.39
End(B)

LC

End(B) 
Annex. 1 - cont.

\begin{tabular}{|c|c|c|c|c|c|c|c|c|c|c|c|}
\hline & FAMILY / Taxon & Life & Flor. element & Herb. & R.84 & R.90 & XK & EUNIS code & EU & END & Note \\
\hline 613. & Veronica officinalis L. & $\mathrm{H}$ & Euro-Asiat. & $\mathrm{N} / \mathrm{A}$ & & - & & $\begin{array}{l}\text { F2.23, F2.231, G1.6933, } \\
\text { G3.1 }\end{array}$ & & & \\
\hline 614. & Veronica orsiniana Ten. & $\mathrm{H}$ & Orof.S.Eur. & L-395 & & & & E4.392, E4.3925 & & & \\
\hline 615. & Veronica persica Poir. & T & Euro-Asiat. & L-396 & - & & & G1.7 & & & \\
\hline 616. & Veronica serpyllifolia L. & $\mathrm{H}$ & Euro-Asiat. & L-397 & & & & E5.51, E5.5721, G1.A1 & & & $\boldsymbol{\nabla}$ \\
\hline \multirow[t]{2}{*}{617.} & Veronica thessalica Benth. & $\mathrm{H}$ & Balkan & L-586 & - & & $C R$ & $\mathrm{H} 2.6, \mathrm{H} 3.2 \mathrm{~A} 13$ & & End(B) & \\
\hline & SCROPHULARIACEAE & & & & & & & & & & \\
\hline 618. & Scrophularia canina L. & $\mathrm{H}$ & Euri-Medit. & N/A & - & & & E1.22, E4.312 & & & \\
\hline 619. & $\begin{array}{l}\text { Scrophularia heterophylla subsp. laciniata (Wald. \& Kit.) Mai. } \\
\text { \& Pet. }\end{array}$ & $\mathrm{H}$ & Balkan & L-343 & & & & $\begin{array}{l}\text { E1.22, H3.2A13, H2.4, } \\
\text { H2.3 }\end{array}$ & & & \\
\hline 620. & Scrophularia nodosa L. & $\mathrm{H}$ & Euro-Siber. & L-344 & - & & & $\begin{array}{l}\text { G1.6933, G4.6, E5.2, } \\
\text { G1.A1 }\end{array}$ & & & \\
\hline 621. & $\begin{array}{l}\text { Verbascum longifolium subsp. pannosum (Vis. \& Pančić) } \\
\text { Murb. }\end{array}$ & $\mathrm{H}$ & Balkan & $\mathrm{N} / \mathrm{A}$ & & - & & E4.4125 & & & \\
\hline 622. & Verbascum nigrum L. & $\mathrm{H}$ & Euro-Asiat. & L-587 & - & & & E1.2, E1.73, E1.74 & & & \\
\hline \multirow[t]{2}{*}{623.} & Verbascum phoeniceum L. & $\mathrm{H}$ & Euro-Asiat. & N/A & - & & & $\mathrm{E} 1.2, \mathrm{G} 1.7, \mathrm{E} 4.5$ & & & \\
\hline & ACANTHACEAE & & & & & & & & & & \\
\hline 624. & $\begin{array}{l}\text { Acanthus hungaricus (Borbás) Baen. } \\
\text { GESNERIACEAE }\end{array}$ & $\mathrm{H}$ & Balkan & L-699 & - & & & G1.7 & & & \\
\hline \multirow[t]{2}{*}{625.} & Ramonda nathaliae Pančić \& Petrovič & $\mathrm{H}$ & Balkan & L-588 & & & EN & H3.2, G1.7C1, H3.2A13 & & End(B) & \\
\hline & LAMIACEAE & & & & & & & & & & \\
\hline 626. & Ajuga chamaepitys (L.) Schreb. & $\mathrm{H}$ & Euro-Asiat. & L-589 & - & & & E1.22 & & & \\
\hline 627. & Ajuga genevensis L. & G & Euro-Asiat. & L-590 & - & & & $\begin{array}{l}\text { E1.22, E1.7, E1.73, } \\
\text { G1.A1 }\end{array}$ & & & \\
\hline 628. & Ajuga laxmannii (Murray) Benth. & $\mathrm{H}$ & Euro-Asiat. & L-591 & - & & & G1.7 & & & \\
\hline 629. & Ajuga pyramidalis $\mathrm{L}$. & $\mathrm{H}$ & Euro-Cauc. & L-281 & - & & & E1.7, E1.92 & & & \\
\hline 630. & Ajuga reptans $\mathrm{L}$. & G & Euro-Asiat. & L-592 & - & & & G1.6933, G1.A1 & & & \\
\hline 631. & Clinopodium acinos (L.) Kunzte & $\mathrm{T}$ & Euri-Medit. & L-282 & - & & & E5.43 & & & \\
\hline 632. & Clinopodium alpinum (L.) Kuntze subsp. alpinum & c & Orof.S.Eur. & L-283 & - & $\bullet$ & & $\begin{array}{l}\text { E4.41, E4.4126, } \\
\text { E4.41731 }\end{array}$ & & & \\
\hline 633. & Clinopodium alpinum subsp. hungaricum (Simonk.) Govaerts & $\mathrm{H}$ & Balkan & N/A & - & & & E4.41731 & & & \\
\hline 634. & Clinopodium grandiflorum (L.) Kuntze & $\mathrm{T}$ & Orof.S.Eur. & L-284 & & - & & F2.231, F2.2, E5.2 & & & \\
\hline 635. & Clinopodium menthifolium (Host) Stace subsp. menthifolium & $\mathrm{H}$ & Euro-Cauc. & N/A & - & & & E5.43 & & & \\
\hline 636. & Clinopodium vulgare L. & $\mathrm{H}$ & Circumbor. & L-285 & & & & E1.22, F3.2421, G1.A1 & & & \\
\hline 637. & Glechoma hirsuta Waldst. \& Kit. & $\mathrm{H}$ & Europ. & $\mathrm{N} / \mathrm{A}$ & - & & & G1.6933, G1.A1 & & & \\
\hline 638. & Lamium amplexicaule L. & $\mathrm{T}$ & Paleotemp. & L-593 & - & & & E5.4 & & & \\
\hline
\end{tabular}


Annex. 1 - cont.

\begin{tabular}{|c|c|c|c|c|c|c|c|c|c|c|c|}
\hline & FAMILY / Taxon & Life & Flor. element & Herb. & R.84 & R.90 & $\mathrm{XK}$ & EUNIS code & EU & END & Note \\
\hline 639. & Lamium galeobdolon (L.) Crantz & $\mathrm{H}$ & Euro-Cauc. & L-286 & - & & & $\begin{array}{l}\text { G1.6933, G4.6, E5.2, } \\
\text { G1.A1 }\end{array}$ & & & \\
\hline 640. & Lamium purpureum $\mathrm{L}$. & $\mathrm{T}$ & Euro-Asiat. & L-594 & - & & & G1.7, E4.413 & & & \\
\hline 641. & Melittis melissophyllum L. & $\mathrm{H}$ & Europ. & L-595 & - & & & $\begin{array}{l}\text { F3.2421, G1.69322, } \\
\text { G1.6933 }\end{array}$ & & & \\
\hline 642. & Mentha longifolia (L.) L. & H & Paleotemp. & L-287 & $\bullet$ & & & $\begin{array}{l}\text { D2.2, D2.22, E5.2, } \\
\text { D2.38, D2.26 }\end{array}$ & & & \\
\hline 643. & Mentha pulegium L. & $\mathrm{H}$ & Medit. & L-713 & & & & E3.4, E5.5 & LC & & \\
\hline 644. & Origanum vulgare $\mathrm{L}$. & $\mathrm{H}$ & Euro-Asiat. & L-288 & & & & E1.2, E1.22, E4.4 & & & \\
\hline 645. & Prunella laciniata (L.) L. & $\mathrm{H}$ & Medit. & N/A & $\bullet$ & & & $\begin{array}{l}\text { E1.22, E1.7, E1.73, } \\
\text { E1.74 }\end{array}$ & & & \\
\hline 646. & Prunella vulgaris $\mathrm{L}$. & $\mathrm{H}$ & Circumbor. & L-289 & $\bullet$ & & & $\begin{array}{l}\text { E1.22, E1.7, E3.4, E5.2, } \\
\text { D2.38 }\end{array}$ & & & \\
\hline 647. & Salvia nemorosa $\mathrm{L}$. & $\mathrm{H}$ & Euro-Asiat. & L-596 & $\bullet$ & & & E1.2, E1.22, F3.2421 & & & \\
\hline 648. & Salvia verticillata $\mathrm{L}$. & H & Euro-Asiat. & L-290 & $\bullet$ & & & $\begin{array}{l}\text { E1.22, E1.7, E3.4, E5.43, } \\
\text { E5.2 }\end{array}$ & & & \\
\hline 649. & Satureja montana L. subsp. montana & c & Medit. & L-291 & & & & E1.22, F3.2421 & & & \\
\hline 650. & Scutellaria alpina L. & $\mathrm{H}$ & Europ. & L-292 & & & EN & $\begin{array}{l}\text { E4.4, H3.2, H3.2A13, } \\
\text { H3.15 }\end{array}$ & & & \\
\hline 651. & Sideritis scardica Griseb. & $\mathrm{H}$ & Balkan & L-293 & & & $C R$ & E1.2, E4.4126, E4.41 & & End(B) & \\
\hline 652. & Stachys alopecuros (L.) Benth. & $\mathrm{H}$ & Europ. & L-294 & & & & F2.231, F2.32, E4.4125 & & & \\
\hline 653. & Stachys alpina L. & $\mathrm{H}$ & Europ. & L-295 & - & & & $\begin{array}{l}\text { E4.413A, E4.39, } \\
\text { E4.3925 }\end{array}$ & & & \\
\hline 654. & Stachys germanica L. & $\mathrm{H}$ & Euri-Medit. & L-296 & - & - & & $\mathrm{E} 1.2, \mathrm{E} 1.22, \mathrm{G} 1.7$ & & & \\
\hline 655. & Stachys officinalis (L.) Trevis. & $\mathrm{H}$ & Euro-Asiat. & L-597 & - & & & $\mathrm{E} 1.22, \mathrm{E} 1.7, \mathrm{E} 1.73$ & & & \\
\hline 656. & Stachys plumosa Griseb. & $\mathrm{H}$ & Balkan & N/A & - & & & E4.39, E4.392 & & End(B) & \\
\hline 657. & Stachys recta $\mathrm{L}$. & $\mathrm{H}$ & Euri-Medit. & L-598 & - & & & E1.2, E1.22, E1.55 & & & \\
\hline 658. & Stachys scardica (Griseb.) Hayek & $\mathrm{H}$ & Balkan & L-297 & $\bullet$ & & & $\mathrm{E} 1.2, \mathrm{G} 1.7, \mathrm{G} 4.6$ & & End(B) & \\
\hline 659. & Stachys sylvatica L. & $\mathrm{H}$ & Euro-Siber. & L-599 & - & & & $\begin{array}{l}\text { G4.6, G1.7, E5.4, } \\
\text { G1.6933, G1.A1 }\end{array}$ & & & \\
\hline 660. & Stachys tymphaea Hausskn. & $\mathrm{H}$ & Medit.-Mont. & N/A & - & & & E4.41 & & & \\
\hline 661. & Teucrium chamaedrys L. & c & Euri-Medit. & L-298 & $\bullet$ & & & $\begin{array}{l}\text { E1.22, E4.41731, E4.42, } \\
\text { E5.2 }\end{array}$ & & & \\
\hline 662. & Teucrium montanum L. & c & Orof.S.Eur. & L-299 & - & - & & E1.55, F3.2421 & & & \\
\hline 663. & Teucrium polium L. & c & Steno-Medit. & N/A & - & & & $F 3.2421$ & & & \\
\hline 664. & Thymus longicaulis C. Presl subsp. Iongicaulis & c & Euri-Medit. & N/A & • & & & E1.22 & & & \\
\hline 665. & $\begin{array}{l}\text { Thymus praecox subsp. zygiformis (Heinr.Braun ex Wett.) } \\
\text { Jalas }\end{array}$ & C & Orof.SE.Eur. & L-327 & $\bullet$ & $\bullet$ & & $\begin{array}{l}\text { E4.3, E4.39, E4.392, } \\
\text { E4.34, H2.4 }\end{array}$ & & End(B) & \\
\hline
\end{tabular}


Annex. 1 - cont.

\begin{tabular}{|c|c|c|c|c|c|c|c|c|c|c|c|}
\hline & FAMILY / Taxon & Life & Flor. element & Herb. & R.84 & R.90 & $\mathrm{XK}$ & EUNIS code & EU & END & Note \\
\hline \multirow[t]{2}{*}{666.} & Thymus pulegioides L. & c & Euro-Asiat. & L-328 & & & & $\begin{array}{l}\text { E1.2, E1.22, E1.74, } \\
\text { F2.231 }\end{array}$ & & & \\
\hline & OROBANCHACEAE & & & & & & & & & & \\
\hline 667. & Euphrasia minima DC. & $\mathrm{T}$ & Orof.C.Eur. & L-600 & • & • & & E4.3, E4.39, E4.3925 & & & \\
\hline 668. & Euphrasia rostkoviana Hayene & $\mathrm{T}$ & Euro-Siber. & L-341 & & & & $\begin{array}{l}\text { E1.71, E1.73, E3.5, } \\
\text { E4.34, E1.112 }\end{array}$ & & & \\
\hline 669. & Euphrasia salisburgensis Funck & $\mathrm{T}$ & Euro-Cauc. & L-601 & $\bullet$ & $\bullet$ & & $\begin{array}{l}\text { E1.2, E1.22, E4.4, } \\
\text { E4.41731 }\end{array}$ & & & \\
\hline 670. & Euphrasia stricta J. F. Lehm. & $\mathrm{T}$ & Europ. & L-700 & $\bullet$ & & & $\begin{array}{l}\text { E1.22, E1.73, E1.74, } \\
\text { F2.263 }\end{array}$ & & & \\
\hline 671. & Melampyrum pratense $\mathrm{L}$. & $\mathrm{T}$ & Euro-Siber. & L-602 & • & & & G1.7, F3.2421 & & & \\
\hline 672. & Melampyrum scardicum Wettst. & $\mathrm{T}$ & Balkan & L-603 & • & & LC & $F 2.22, F 2.231, F 2.3$ & & End(B) & \\
\hline 673. & Orobanche caryophyllacea Sm. & $\mathrm{T}$ & Euro-Asiat. & L-359 & & & & E1.22, E1.73 & & & \\
\hline 674. & Parentucellia latifolia (L.) Caruel & $\mathrm{T}$ & Euri-Medit. & N/A & • & & & E1.92 & & & \\
\hline 675. & Pedicularis brachyodonta subsp. grisebachii (Wettst.) Hayek & $\mathrm{H}$ & Balkan & L-604 & & • & LC & E4.412, E4.4122 & & $\operatorname{End}(B)$ & \\
\hline 676. & Pedicularis friderici-augusti Tomm. & $\mathrm{H}$ & Medit.-Mont. & N/A & & • & & E1.22 & & & \\
\hline 677. & Pedicularis oederi Vahl & $\mathrm{H}$ & Circumbor. & N/A & • & & & E4.39, E4.392 & & & \\
\hline 678. & Pedicularis verticillata $\mathrm{L}$. & $\mathrm{H}$ & Art.Alp. & L-605 & $\bullet$ & - & & E4.3, E4.39, E4.392 & & & \\
\hline 679. & Rhinanthus minor $\mathrm{L}$. & $\mathrm{T}$ & Circumbor. & L-606 & • & & & $\mathrm{E} 1.2, \mathrm{E} 1.7, \mathrm{E} 1.73, \mathrm{E} 1.92$ & & & \\
\hline \multirow[t]{2}{*}{680.} & Rhinanthus rumelicus Velen. & $\mathrm{T}$ & Medit.-Mont. & N/A & - & & & $\mathrm{E} 1.2, \mathrm{E} 1.7, \mathrm{E} 1.73$ & & & \\
\hline & VERBENACEAE & & & & & & & & & & \\
\hline 681. & Verbena officinalis L. & $\mathrm{H}$ & Paleotemp. & L-361 & & & & E3.4, E5.3, E5.4, E5.2 & & & \\
\hline \multirow[t]{2}{*}{682.} & Pinguicula balcanica Casper. & $\mathrm{H}$ & Balkan & L-177 & - & & NT & $\begin{array}{l}\text { D2.22, D2.26, D2.282. } \\
\text { D2.38, D } 4.2\end{array}$ & & $\operatorname{End}(B)$ & \\
\hline & CONVOLVULACEAE & & & & & & & & & & \\
\hline 683. & Convolvulus arvensis $\mathrm{L}$. & G & Paleotemp. & L-607 & • & & & $\mathrm{E} 1.2, \mathrm{E} 1.7, \mathrm{E} 1.73, \mathrm{E} 1.92$ & & & \\
\hline 684. & Convolvulus boissieri subsp. compactus (Boiss.) Stace & c & Balkan & N/A & - & & & E1.2 & & $\operatorname{End}(B)$ & \\
\hline 685. & $\begin{array}{l}\text { Convolvulus cantabrica L. } \\
\text { SOLANACEAE }\end{array}$ & $\mathrm{H}$ & Euri-Medit. & L-608 & - & & & $\mathrm{E} 1.2, \mathrm{E} 1.55$ & & & \\
\hline 686. & Atropa bella-donna $\mathrm{L}$. & $\mathrm{H}$ & Orof.S.Eur. & L-354 & - & & & G1.6924, E5.4, E5.2 & & & \\
\hline 687. & $\begin{array}{l}\text { Datura stramonium L. } \\
\text { BORAGINACEAE }\end{array}$ & $\mathrm{T}$ & Cosmopol. & L-609 & $\bullet$ & & & E5.5, E5.51 & & & \\
\hline 688. & Buglossoides arvensis (L.) I. M. Johnst. & $\mathrm{T}$ & Euri-Medit. & N/A & - & & & E5.4 & & & \\
\hline 689. & Cerinthe minor $\mathrm{L}$. & $\mathrm{T}$ & Euro-Siber. & L-610 & $\bullet$ & & & $\begin{array}{l}\text { E1.7, E1.76, E1.74, } \\
\text { E1.92 }\end{array}$ & & & \\
\hline 690. & Myosotis alpestris Hoffm. & $\mathrm{H}$ & Euro-Asiat. & L-329 & • & • & & E4.12, E4.3, E4.39, E4.5 & & & \\
\hline
\end{tabular}


Annex. 1 - cont.

\begin{tabular}{|c|c|c|c|c|c|c|c|c|c|c|c|}
\hline & FAMILY / Taxon & Life & Flor. element & Herb. & R.84 & R.90 & XK & EUNIS code & EU & END & Note \\
\hline 691. & Myosotis suaveolens Willd. & $\mathrm{H}$ & Balkan & L-330 & & & & $\mathrm{F} 2.231, \mathrm{~F} 2.263, \mathrm{H} 2.4$ & & End(B) & \\
\hline 692. & Myosotis sylvatica Hoffm. & $\mathrm{H}$ & Paleotemp. & L-331 & - & & & $\begin{array}{l}\text { G1.6933, E5.43, G3.1, } \\
\text { G1.A1 }\end{array}$ & & & \\
\hline 693. & Pulmonaria officinalis L. & $\mathrm{H}$ & Europ. & L-333 & $\bullet$ & & & $\begin{array}{l}\text { G4.6, G1.6933, E5.43, } \\
\text { E5.2, G1.A1 }\end{array}$ & & & \\
\hline 694. & Pulmonaria rubra Schott. & $\mathrm{H}$ & Balkan & L-334 & & & & E5.43, G1.6933 & & & \\
\hline 695. & $\begin{array}{l}\text { Symphytum tuberosum L. } \\
\text { ARALIACEAE }\end{array}$ & G & Euri-Medit. & L-611 & - & - & & $\mathrm{E} 4.3, \mathrm{G} 4.6, \mathrm{E} 5.43, \mathrm{G} 3.1$ & & & \\
\hline 696. & Hedera helix L. & $\mathrm{P}$ & Euri-Medit. & L-335 & $\bullet$ & & & $\begin{array}{l}\text { F3.2421, G4.6, E5.2, } \\
\text { G1.A1 }\end{array}$ & & & \\
\hline & APIACEAE & & & & & & & & & & \\
\hline 697. & Aegopodium podagraria $\mathrm{L}$. & G & Euro-Siber. & L-401 & $\bullet$ & & & $\begin{array}{l}\text { E3.4, E3.5, F2.231, E5.2, } \\
\text { G1.A1 }\end{array}$ & & & \\
\hline 698. & Angelica sylvestris L. & $\mathrm{H}$ & Euro-Siber. & L-402 & & & & E4.412, G3.12 & & & \\
\hline 699. & Anthriscus caucalis M. Bieb. & T & Euri-Medit. & L-403 & & & & E5.43 & & & \\
\hline 700. & Astrantia major L. & $\mathrm{H}$ & Euro-Asiat. & L-612 & $\bullet$ & & & $\begin{array}{l}\text { E4.4126, E4.41731, } \\
\text { E4.4122 }\end{array}$ & & & \\
\hline 701. & Bifora radians M. Bieb. & $\mathrm{T}$ & Euro-Asiat. & N/A & - & & & E3.4, E5.43 & & & \\
\hline 702. & Bupleurum falcatum subsp. cernuum (Ten.) Arc. & $\mathrm{H}$ & Euro-Siber. & L-404 & - & & & E4.41 & & & \\
\hline 703. & Bupleurum karglii Vis. & T & Balkan & L-405 & - & & & E4.312, E4.392, E4.412 & & End(B) & \\
\hline 704. & Bupleurum ranunculoides $\mathrm{L}$. & $\mathrm{H}$ & Circumbor. & L-613 & & - & & E4.4, E4.41, E4.4121 & & & \\
\hline 705. & Bupleurum veronense Turra & T & Orof.SE.Eur. & N/A & - & & & $\mathrm{E} 1.2, \mathrm{E} 1.55$ & & & \\
\hline 706. & Chaerophyllum aromaticum L. & $\mathrm{H}$ & Europ. & L-406 & & & & $\mathrm{E} 5.4, \mathrm{G} 1.7, \mathrm{E} 1.2$ & & & \\
\hline 707. & Chaerophyllum aureum L. & $\mathrm{H}$ & Euri-Medit. & L-407 & & & & $\mathrm{E} 1.2, \mathrm{E} 1.22$ & & & \\
\hline 708. & Chaerophyllum hirsutum L. & $\mathrm{H}$ & Europ. & L-408 & & & & E5.4, E5.43, E5.5, E5.2 & & & \\
\hline 709. & Daucus carota $\mathrm{L}$. & $\mathrm{T}$ & Paleotemp. & L-614 & - & & & E5.4 & & & \\
\hline 710. & Eryngium campestre $\mathrm{L}$. & $\mathrm{H}$ & Euro-Asiat. & L-615 & - & & & F3.2421 & & & \\
\hline 711. & Eryngium palmatum Pančić \& Vis. & $\mathrm{H}$ & Balkan & N/A & - & & & G1.7 & & End(B) & \\
\hline 712. & Laser trilobum (L.) Borkh. & H & Euro-Asiat. & L-616 & $\bullet$ & & & G1.7 & & & \\
\hline 713. & Ligusticum mutellina (L.) Crantz & $\mathrm{H}$ & Orof.S.Eur. & L-409 & - & - & & E1.7, E4.3, E4.39, E4.34 & & & \\
\hline 714. & Meum athamanticum Jacq. & $\mathrm{H}$ & Europ. & L-410 & & & & E4.39, E4.392 & & & \\
\hline 715. & Oenanthe fistulosa $\mathrm{L}$. & $\mathrm{H}$ & Euro-Asiat. & N/A & - & & & D5.131 & LC & & \\
\hline 716. & Oenanthe peucedanifolia Pollich. & $\mathrm{H}$ & Medit. & L-411 & - & & & E4.116, E4.5 & & & \\
\hline 717. & Oenanthe silaifolia M. Bieb. & $\mathrm{H}$ & Euro-Asiat. & N/A & - & & & E5.4, E5.5721 & & & \\
\hline 718. & Orlaya grandiflora (L.) Hoffm. & $\mathrm{T}$ & Euro-Asiat. & L-617 & - & & & E1.22, E1.55, E5.4 & & & \\
\hline 719. & Physospermum cornubiense (L.) DC. & H & Euro-Asiat. & L-412 & & & & G1.7 & & & \\
\hline 720. & Pimpinella alpina Koord. & $\mathrm{H}$ & Orof.S.Eur. & L-413 & - & & & $\mathrm{E} 4.3, \mathrm{E} 4.312$ & & & \\
\hline
\end{tabular}


Annex. 1 - cont.

\begin{tabular}{|c|c|c|c|c|c|c|c|c|c|c|c|}
\hline & FAMILY / Taxon & Life & Flor. element & Herb. & R.84 & R.90 & XK & EUNIS code & EU & END & Note \\
\hline 721. & Pimpinella saxifraga $\mathrm{L}$. & $\mathrm{H}$ & Euro-Cauc. & L-414 & $\bullet$ & $\bullet$ & & $\begin{array}{l}\text { E1.2, E4.3, E4.312, } \\
\text { E4.34 }\end{array}$ & & & \\
\hline 722. & Sanicula europaea L. & $\mathrm{H}$ & Euro-Siber. & L-618 & $\bullet$ & & & $\begin{array}{l}\text { G1.7, G1.6933, G4.6, } \\
\text { G3.1, G1.A1 }\end{array}$ & & & \\
\hline 723. & Scandix pecten-veneris $\mathrm{L}$. & $\mathrm{T}$ & Euri-Medit. & N/A & $\bullet$ & & & E5.43 & & & \\
\hline 724. & Seseli montanum L. & $\mathrm{H}$ & Medit.-Mont. & L-619 & $\bullet$ & & & F3.2421, H3.1 & & & \\
\hline 725. & Smyrnium perfoliatum L. & $\mathrm{H}$ & Euri-Medit. & N/A & $\bullet$ & & & F3.2421, G1.6933 & & & \\
\hline 726. & Trinia dalechampii (Ten.) Janch. & $\mathrm{H}$ & Orof.S.Eur. & L-416 & $\bullet$ & & & E4.41, E4.4126, E4.34 & & & \\
\hline \multirow[t]{2}{*}{727.} & Trinia glauca (L.) Dumort & $\mathrm{H}$ & Europ. & L-417 & $\bullet$ & $\bullet$ & & $\mathrm{F} 2.2, \mathrm{~F} 2.23$ & & & \\
\hline & VIBURNACEAE & & & & & & & & & & \\
\hline 728. & Sambucus ebulus L. & G & Euri-Medit. & L-238 & $\bullet$ & & & $\begin{array}{l}\text { E4.1, F3.2421, G1.7, } \\
\text { G4.6, E5.2 }\end{array}$ & & & \\
\hline 729. & Sambucus nigra L. & $\mathrm{P}$ & Euro-Asiat. & L-239 & $\bullet$ & & & $\mathrm{E} 5.43, \mathrm{E} 5.5, \mathrm{E} 5.2, \mathrm{G} 1 . \mathrm{A} 1$ & & & \\
\hline \multirow[t]{2}{*}{730.} & Sambucus racemosa $\mathrm{L}$. & $P$ & Euro-Asiat. & $\mathrm{L}-240$ & $\bullet$ & & & G4.6, E5.43, E5.2 & & & \\
\hline & CAPRIFOLIACEAE & & & & & & & & & & \\
\hline 731. & Lonicera alpigena $\mathrm{L}$. & $P$ & Orof.S.Eur. & L-263 & $\bullet$ & & & G1.6933, G4.6, E5.2 & & & \\
\hline 732. & Lonicera caprifolium L. & $\mathrm{P}$ & Europ. & L-620 & $\bullet$ & & & G4.6, G1.A1 & & & \\
\hline \multirow[t]{2}{*}{733.} & Viburnum lantana L. & C & Europ. & L-621 & $\bullet$ & & & F3.2421, G1.A1 & & & \\
\hline & DIPSACACEAE & & & & & & & & & & \\
\hline 734. & Cephalaria leucantha (L.) Roem. \& Schult. & $\mathrm{H}$ & Medit.-Mont. & L-622 & $\bullet$ & & & E1.2 & & & \\
\hline 735. & Dipsacus fullonum L. & $\mathrm{H}$ & Euri-Medit. & N/A & $\bullet$ & & & E5.43 & & & \\
\hline 736. & Knautia arvensis (L.) DC. & $\mathrm{H}$ & Euro-Asiat. & L-193 & $\bullet$ & $\bullet$ & & $\mathrm{E} 1.2, \mathrm{E} 1.22$ & & & \\
\hline 737. & Knautia drymeia Heuff. & $\mathrm{H}$ & Europ. & N/A & $\bullet$ & & & G1.6933, G4.6, E4.312 & & & \\
\hline 738. & Knautia midzorensis Formánek & $\mathrm{H}$ & Balkan & L-646 & $\bullet$ & $\bullet$ & EN & E4.4, E4.41, E4.4122 & & End(B) & \\
\hline 739. & Scabiosa columbaria L. & $\mathrm{T}$ & Euro-Asiat. & L-194 & $\bullet$ & & & $\begin{array}{l}\text { F2.2, E4.392, E4.3925, } \\
\text { E4.41 }\end{array}$ & & & \\
\hline \multirow[t]{2}{*}{740.} & Scabiosa taygetea subsp. portae (Huter) Kokkini & $\mathrm{H}$ & Balkan & L-645 & $\bullet$ & $\bullet$ & & E4.312, F2.231 & & End(B) & \\
\hline & VALERIANACEAE & & & & & & & & & & \\
\hline 741. & Valeriana montana Griseb. & $\mathrm{H}$ & Orof.S.Eur. & L-236 & & & & E5.5, E5.51, H3.15 & & & \\
\hline \multirow[t]{2}{*}{742.} & Valeriana officinalis L. & $\mathrm{H}$ & Europ. & L-237 & & & & E1.74, E1.92, E3.4 & & & \\
\hline & CAMPANULACEAE & & & & & & & & & & \\
\hline 743. & Asyneuma limonifolium (L.) Janch. & $\mathrm{H}$ & Euri-Medit. & $L-429$ & $\bullet$ & & & E1.5, E1.7 & & & \\
\hline 744. & Campanula abietina Griseb. & $\mathrm{H}$ & Balkan & L-716 & & & & E1.7 & DD & & \\
\hline 745. & Campanula alpina Jacq. & $\mathrm{H}$ & Orof.SE.Eur. & L-623 & $\bullet$ & $\bullet$ & LC & E4.392, E4.3925, F2.11 & & & \\
\hline 746. & Campanula foliosa Ten. & $\mathrm{H}$ & Orof.SE.Eur. & L-430 & & $\bullet$ & LC & F2.263, F2.231, E5.43 & & & \\
\hline 747. & Campanula glomerata L. & $\mathrm{H}$ & Euro-Asiat. & L-431 & $\bullet$ & & & E4.4, E1.2, E1.22, E1.7 & & & \\
\hline 748. & Campanula patula $\mathrm{L}$. & $\mathrm{H}$ & Euro-Asiat. & L-433 & & & & E1.2, E1.7, E1.73, E1.74 & & & \\
\hline
\end{tabular}


Annex. 1 - cont.

\begin{tabular}{|c|c|c|c|c|c|c|c|c|c|c|c|}
\hline & FAMILY / Taxon & Life & Flor. element & Herb. & R.84 & R.90 & XK & EUNIS code & EU & END & Note \\
\hline 749. & Campanula persicifolia $\mathrm{L}$. & $\mathrm{H}$ & Euro-Siber. & L-434 & $\bullet$ & & & $\begin{array}{l}\text { F3.2421, G1.6933, E5.2, } \\
\text { G1.A1 }\end{array}$ & & & \\
\hline 750. & Campanula rotundifolia $\mathrm{L}$. & $\mathrm{H}$ & Europ. & L-435 & $\bullet$ & $\bullet$ & EN & $\begin{array}{l}\text { E4.3, E4.312, E4.39, } \\
\text { E4.34, H2.4 }\end{array}$ & & & \\
\hline 751. & Campanula scheuchzeri Vill. & $\mathrm{H}$ & Orof.S.Eur. & L-644 & $\bullet$ & $\bullet$ & & E1.7, E1.73, E4.3, E4.39 & & & \\
\hline 752. & Campanula spatulata Sm. subsp. spatulata & G & Balkan & L-624 & & $\bullet$ & & E4.39 & & End(B) & \\
\hline 753. & Campanula trachelium $\mathrm{L}$. & $\mathrm{H}$ & Paleotemp. & L-437 & $\bullet$ & & & $\begin{array}{l}\text { G1.6933, E5.43, E5.2, } \\
\text { G1.A1 }\end{array}$ & & & \\
\hline 754. & Edraianthus graminifolius (L.) A.DC. & $\mathrm{C}$ & Balkan & L-438 & $\bullet$ & $\bullet$ & & E4.4, E4.412, E1.112 & & & \\
\hline 755. & Jasione orbiculata Griseb. & $\mathrm{H}$ & Balkan & L-439 & $\bullet$ & $\bullet$ & & $\begin{array}{l}\text { F2.2, E1.7, E4.3, E4.39, } \\
\text { E4.34 }\end{array}$ & & & \\
\hline 756. & Phyteuma confusum A. Kern. & $\mathrm{H}$ & Orof.S.Eur. & L-625 & $\bullet$ & $\bullet$ & & E4.3, E4.39, E4.3925 & & & \\
\hline 757. & Phyteuma orbiculare L. & $\mathrm{H}$ & Orof.S.Eur. & N/A & $\bullet$ & & & $\mathrm{E} 4.3, \mathrm{E} 4.392$ & & & \\
\hline 758. & $\begin{array}{l}\text { Phyteuma pseudorbiculare Pant. } \\
\text { ASTERACEAE }\end{array}$ & $\mathrm{H}$ & Balkan & L-440 & & $\bullet$ & LC & E4.3, E4.39 & & End(B) & \\
\hline 759. & Achillea abrotanoides (Vis.) Vis & C & Balkan & L-355 & & $\bullet$ & & $\mathrm{F} 2.32, \mathrm{H} 3.2 \mathrm{~A} 13$ & & End(B) & \\
\hline 760. & $\begin{array}{l}\text { Achillea ageratifolia (Sm.) Benth. \& Hook. f. subsp. } \\
\text { ageratifolia }\end{array}$ & $\mathrm{H}$ & Balkan & L-565 & & & & $\mathrm{E} 4.41, \mathrm{H} 2.4$ & & End(B) & \\
\hline 761. & Achillea chrysocoma Friv. & $\mathrm{H}$ & Balkan & L-358 & $\bullet$ & $\bullet$ & EN & E4.41, E4.4126, F2.231 & & End(B) & \\
\hline 762. & Achillea clusiana Tausch & $\mathrm{H}$ & Balkan & L-363 & & & & $F 2.22, F 2.263$ & & & \\
\hline 763. & Achillea coarctata Poir. & $\mathrm{H}$ & Europ. & N/A & $\bullet$ & & & $\mathrm{E} 1.2, \mathrm{E} 1.22$ & & & \\
\hline 764. & Achillea distans subsp. tanacetifolia (Fiori) Janch. & $\mathrm{H}$ & Orof.S.Eur. & N/A & & $\bullet$ & & E5.4 & & & \\
\hline 765. & Achillea millefolium $\mathrm{L}$. & $\mathrm{H}$ & Euro-Siber. & L-362 & $\bullet$ & $\bullet$ & & $\mathrm{E} 5.43, \mathrm{E} 4.413, \mathrm{E} 4.413 \mathrm{~A}$ & & & \\
\hline 766. & Achillea nobilis L. & $\mathrm{H}$ & Euro-Asiat. & N/A & $\bullet$ & & & E1.2, E1.7 & & & \\
\hline 767. & Achillea setacea Waldst. et Kit. & $\mathrm{H}$ & Euro-Asiat. & L-441 & & & & $\mathrm{E} 1.2, \mathrm{E} 1.7, \mathrm{E} 1.92$ & & & \\
\hline 768. & Adenostyles alliariae (Gouan) A. Kern. & $\mathrm{H}$ & Europ. & L-626 & $\bullet$ & & & G1.6933, F2.11, E5.43 & & & \\
\hline 769. & Antennaria dioica (L.) Gaertn. & C & Circumbor. & L-442 & $\bullet$ & $\bullet$ & & $\begin{array}{l}\text { E1.71, E4.3, E4.39, } \\
\text { E4.34, E1.112 }\end{array}$ & & & \\
\hline 770. & Anthemis arvensis L. & $\mathrm{T}$ & Euro-Asiat. & L-627 & $\bullet$ & & & E1.2 & & & \\
\hline 771. & Anthemis cretica subsp. carpatica (Willd.) Grierson & $\mathrm{H}$ & Europ. & L-717 & & & & E4.3, E4.39, E4.3922 & & & \\
\hline 772. & Arctium lappa $\mathrm{L}$. & $\mathrm{H}$ & Euro-Asiat. & $\mathrm{L}-443$ & & & & E5.43, E5.5, E5.2 & & & \\
\hline 773. & $\begin{array}{l}\text { Artemisia umbelliformis subsp. eriantha (Ten.) Vallès-Xirau \& } \\
\text { Oliva Brañas }\end{array}$ & $\mathrm{C}$ & Orof.S.Eur. & L-444 & & & EN & E4.3925, H3.15, H3.152 & & & \\
\hline 774. & Artemisia vulgaris $\mathrm{L}$. & $\mathrm{H}$ & Circumbor. & L-445 & & & & $\mathrm{E} 5.43, \mathrm{E} 5.2$ & & & \\
\hline 775. & Aster alpinus L. & $\mathrm{H}$ & Euro-Asiat. & L-446 & $\bullet$ & $\bullet$ & & $\begin{array}{l}\text { E4.4, E4.41, E4.4121, } \\
\text { H2.4 }\end{array}$ & & & \\
\hline
\end{tabular}


Annex. 1 - cont.

\begin{tabular}{|c|c|c|c|c|c|c|c|c|c|c|c|}
\hline & FAMILY / Taxon & Life & Flor. element & Herb. & R.84 & R.90 & $\mathrm{XK}$ & EUNIS code & EU & END & Note \\
\hline 776. & Bellis perennis $\mathrm{L}$. & $\mathrm{H}$ & Euro-Asiat. & L-447 & $\bullet$ & & & $\begin{array}{l}\text { E1.2, E1.22, E4.312, } \\
\text { E4.39 }\end{array}$ & & & \\
\hline 777. & Carduus acanthoides L. & $\mathrm{H}$ & Euro-Asiat. & $\mathrm{L}-421$ & & & & $\mathrm{E} 1.2, \mathrm{E} 4.34$ & & & \\
\hline 778. & Carduus candicans Wald et Kit. & $\mathrm{H}$ & Balkan & L-422 & $\bullet$ & & & E4.392, F2.231, E1.2 & & & \\
\hline 779. & Carduus carduelis (L.) Gren. & $\mathrm{H}$ & Orof.S.Eur. & N/A & & $\bullet$ & & E4.3, E4.39, E4.392 & & & \\
\hline 780. & Carlina acaulis L. & $\mathrm{H}$ & Europ. & L-374 & & & & $\begin{array}{l}\text { E1.2, E1.22, E1.74, } \\
\text { E1.92, E4.34 }\end{array}$ & & & \\
\hline 781. & Centaurea jacea L. & $\mathrm{H}$ & Euro-Siber. & $\mathrm{N} / \mathrm{A}$ & $\bullet$ & & & $\mathrm{E} 1.2, \mathrm{E} 1.22, \mathrm{E} 1.92$ & & & \\
\hline 782. & Centaurea nervosa Willd. & $\mathrm{H}$ & Orof.SE.Eur. & L-628 & $\bullet$ & & & E4.3, E4.31, E4.39 & & & \\
\hline 783. & Cirsium appendiculatum Griseb. & $\mathrm{H}$ & Balkan & L-398 & $\bullet$ & & & E5.57, E5.5721 & & End(B) & \\
\hline 784. & Cirsium arvense (L.) Scop. & $\mathrm{H}$ & Euro-Asiat. & L-399 & • & & & $\begin{array}{l}\text { E5.43, E3.4, F2.231, } \\
\text { E5.2, G1.1 }\end{array}$ & & & \\
\hline 785. & Cirsium canum (L.) All. & G & Orof.S.Eur. & N/A & $\bullet$ & & & E3.4 & & & \\
\hline 786. & Cirsium eriophorum (L.) Scop. & $\mathrm{H}$ & Europ. & $\mathrm{L}-400$ & $\bullet$ & & & $\mathrm{E} 1.22, \mathrm{E} 1.7, \mathrm{E} 1.71$ & & & \\
\hline 787. & Crepis aurea subsp. glabrescens (Caurel.) Ar. & $\mathrm{H}$ & Orof.S.Eur. & L-424 & & & & $\mathrm{E} 4.412$ & & & \\
\hline 788. & Crepis viscidula Froel. & $\mathrm{H}$ & Balkan & N/A & & $\bullet$ & & $\mathrm{E} 4.3, \mathrm{E} 4.39$ & & & \\
\hline 789. & Crupina vulgaris Cass. & $\mathrm{T}$ & Euro-Asiat. & L-629 & $\bullet$ & & & E1.7 & & & \\
\hline 790. & Cyanus segetum Hill & $\mathrm{T}$ & Euri-Medit. & N/A & $\bullet$ & & & $\mathrm{E} 5.43, \mathrm{E} 5.5$ & & & \\
\hline 791. & Cyanus triumfettii subsp. axillaris (Čelak.) Štěpánek & $\mathrm{H}$ & Euro-Asiat. & L-373 & $\bullet$ & $\bullet$ & & E4.41, E4.412 & & & \\
\hline 792. & Doronicum austriacum Jacq. & G & Orof.S.Eur. & L-418 & $\bullet$ & & & $\begin{array}{l}\text { E5.57, E5.5721, } \\
\text { G1.6933 }\end{array}$ & & & \\
\hline 793. & Doronicum columnae Ten. & G & Orof.SE.Eur. & L-419 & $\bullet$ & $\bullet$ & & $\begin{array}{l}\text { E4.3, E4.39, E4.392, } \\
\text { F2.32 }\end{array}$ & & & \\
\hline 794. & Erigeron alpinus L. & $\mathrm{H}$ & Euro-Asiat. & L-425 & $\bullet$ & & & H3.152 & & & \\
\hline 795. & Erigeron annuus (L.) Desf. & $\mathrm{T}$ & N. Amer. & L-426 & & & & $\mathrm{E} 5.43, \mathrm{E} 5.2$ & & & \\
\hline 796. & Erigeron glabratus Bluff \& Fingerh. & $\mathrm{H}$ & Medit.-Mont. & L-630 & & $\bullet$ & & E4.391, E4.392 & & & \\
\hline 797. & Eupatorium cannabinum L. & $\mathrm{H}$ & Paleotemp. & $\mathrm{L}-420$ & & & & G1.6933, G4.6, E5.2 & & & \\
\hline 798. & Filago germanica (L.) Huds. & $\mathrm{T}$ & Paleotemp. & N/A & $\bullet$ & & & E1.92 & & & \\
\hline 799. & Galatella linosyris (L.) Rchb. f. & $\mathrm{H}$ & Paleotemp. & N/A & $\bullet$ & & & E1.92 & & & \\
\hline 800. & Gnaphalium supinum L. & $\mathrm{H}$ & Arctic-Alp. & L-631 & $\bullet$ & & & E4.1, E4.116, E4.5 & & & \\
\hline 801. & Hieracium bosniacum Freyn & $\mathrm{H}$ & Balkan & L-722 & & & & E4.412, E4.4121 & & End(B) & \\
\hline 802. & Hieracium murorum L. & $\mathrm{H}$ & Euro-Asiat. & L-457 & & $\bullet$ & & $\begin{array}{l}\text { E5.43, G4.6, G1.6933, } \\
\text { E5.2, G1.A1 }\end{array}$ & & & \\
\hline 803. & Hieracium naegelianum Pančić subsp. naegelianum & $\mathrm{H}$ & Medit.-Mont. & L-458 & & & & E4.392, E4.3925 & & & \\
\hline 804. & Hieracium naegelianum subsp. ljubotenicum O. Behr \& al. & $\mathrm{H}$ & Balkan & L-719 & & & & E4.3411 & & End(B) & \\
\hline 805. & Hieracium piliferum Hoppe & $\mathrm{H}$ & Orof.SE.Eur. & N/A & $\bullet$ & & & E4.41 & & & \\
\hline 806. & Hieracium pilosum Froel. subsp. pilosum & $\mathrm{H}$ & Orof.SE.Eur. & L-459 & & & & E4.412 & & & \\
\hline
\end{tabular}


Annex. 1 - cont.

\begin{tabular}{|c|c|c|c|c|c|c|c|c|c|c|c|}
\hline & FAMILY / Taxon & Life & Flor. element & Herb. & R.84 & R.90 & $\mathrm{XK}$ & EUNIS code & EU & END & Note \\
\hline 807. & Hieracium pilosum subsp. villosiceps Gottschl. & $\mathrm{H}$ & Orof. S-Europ. & L-723 & & & & E4.3411 & & & $\boldsymbol{\nabla}$ \\
\hline 808. & Hieracium sparsum Friv. & $\mathrm{H}$ & Euro-Asiat. & N/A & & $\bullet$ & & E4.4121 & & & \\
\hline 809. & Hieracium villosum Jacq. & $\mathrm{H}$ & Orof.SE.Eur. & L-460 & & & & $\begin{array}{l}\text { E1.7, E1.74, E1.92, } \\
\text { H3.2, H2.4 }\end{array}$ & & & \\
\hline 810. & Homogyne alpina (L.) Cast. & $\mathrm{H}$ & Europ. & L-454 & $\bullet$ & $\bullet$ & & $\begin{array}{l}\text { E4.39, E4.4125, } \\
\text { H3.2A13 }\end{array}$ & & & \\
\hline 811. & Inula ensifolia L. & $\mathrm{H}$ & Europ. & L-632 & $\bullet$ & & & E1.2, E1.71, E1.74, H2.6 & & & \\
\hline 812. & Inula oculus-christi L. & $\mathrm{H}$ & Euro-Asiat. & N/A & $\bullet$ & & & E1.92 & & & \\
\hline 813. & Inula salicina $\mathrm{L}$. & $\mathrm{H}$ & Euro-Asiat. & L-633 & $\bullet$ & & & E1.7, E1.74, E3.3 & & & \\
\hline 814. & $\begin{array}{l}\text { Jacobaea abrotanifolia subsp. carpathica (Herbich) B. Nord. } \\
\text { \& Greuter }\end{array}$ & $\mathrm{C}$ & Medit.-Mont. & L-643 & $\bullet$ & $\bullet$ & & E4.39, E4.391 & & & \\
\hline 815. & Lactuca muralis (L.) Gaertn. & $\mathrm{H}$ & Euro-Cauc. & L-451 & $\bullet$ & & & $\begin{array}{l}\text { G1.6933, G4.6, E5.4, } \\
\text { E5.2, G1.A1 }\end{array}$ & LC & & \\
\hline 816. & Lapsana communis L. & $\mathrm{T}$ & Paleotemp. & L-452 & $\bullet$ & & & $\begin{array}{l}\text { G1.6933, E5.4, E5.2, } \\
\text { G1.A1 }\end{array}$ & & & \\
\hline 817. & Leontodon biscutellifolius DC. & $\mathrm{H}$ & Euro-Asiat. & N/A & $\bullet$ & & & $\mathrm{E} 1.22, \mathrm{E} 4.41$ & & & \\
\hline 818. & Leontodon crispus Vill. & $\mathrm{H}$ & Orof.S.Eur. & L-427 & & & & $\mathrm{E} 1.22, \mathrm{E} 4.5, \mathrm{G} 1.7, \mathrm{H} 2.4$ & & & \\
\hline 819. & Leontodon hispidus L. & $\mathrm{H}$ & Euro-Cauc. & L-428 & $\bullet$ & & & E1.2, E1.7, E1.73, E1.74 & & & \\
\hline 820. & Leucanthemum vulgare (Vaill.) Lam. & $\mathrm{H}$ & Euro-Siber. & L-463 & $\bullet$ & & & $\mathrm{E} 1.2, \mathrm{E} 1.72, \mathrm{E} 1.73, \mathrm{E} 5.4$ & & & \\
\hline 821. & Matricaria chamomilla L. & $\mathrm{T}$ & Cosmopol. & L-634 & $\bullet$ & & & E5.43 & & & \\
\hline 822. & Petasites albus (L.) Gaertn. & G & Europ. & L-464 & & & & $\begin{array}{l}\text { G1.6933, G1.7, G4.6, } \\
\text { E5.43 }\end{array}$ & & & \\
\hline 823. & Pilosella acutifolia (Vill.) Arv.-Touv. & $\mathrm{H}$ & Europ. & L-455 & & & & $\mathrm{F} 2.11, \mathrm{~F} 2.12$ & & & \\
\hline 824. & Pilosella alpicola (Hoppe) F. W. Schultz \& Sch. Bip. & $\mathrm{H}$ & Orof.S.Eur. & L-635 & $\bullet$ & & & $F 2.231, F 2.263, F 2.22$ & & & \\
\hline 825. & Pilosella cymosa (L.) F. W. Schultz \& Sch. Bip. & $\mathrm{H}$ & Europ. & L-465 & $\bullet$ & & & $\mathrm{E} 1.22, \mathrm{E} 1.71, \mathrm{E} 4.39$ & & & \\
\hline 826. & Pilosella guthnikiana (Hegetschw.) Soják & $\mathrm{H}$ & Euro-Asiat. & N/A & & $\bullet$ & & E4.3411 & & & \\
\hline 827. & Pilosella hoppeana (Schult.) F. W. Schultz \& Sch. Bip. & $\mathrm{H}$ & Medit.-Mont. & L-456 & & & & $\begin{array}{l}\text { E1.2, E1.92, E1.22, E1.7, } \\
\text { E4.34 }\end{array}$ & & & \\
\hline 828. & Pilosella macrotricha (Boiss.) F. W. Schultz \& Sch. Bip. & $\mathrm{H}$ & Euro-Asiat. & L-724 & & & & E4.4122 & & & घ \\
\hline 829. & Pilosella officinarum Vaill. & $\mathrm{H}$ & Euro-Cauc. & L-466 & $\bullet$ & $\bullet$ & & $\begin{array}{l}\text { E1.2, E1.22, E1.55, } \\
\text { E4.34 }\end{array}$ & & & \\
\hline 830. & Podospermum laciniatum (L.) DC. & $\mathrm{T}$ & Paleotemp. & N/A & $\bullet$ & & & $\mathrm{E} 1.22, \mathrm{E} 4.41$ & & & \\
\hline 831. & $\begin{array}{l}\text { Podospermum roseum (Waldst. \& Kit.) Gemeinholzer \& } \\
\text { Greuter }\end{array}$ & $\mathrm{H}$ & Europ. & $\mathrm{N} / \mathrm{A}$ & $\bullet$ & & & F3.2421 & & & \\
\hline 832. & Prenanthes purpurea $\mathrm{L}$. & $\mathrm{H}$ & Euro-Asiat. & L-636 & $\bullet$ & & & G1.7, G4.6 & & & \\
\hline 833. & Ptilostemon afer (Jacq.) Greuter & $\mathrm{H}$ & Balkan & L-467 & & $\bullet$ & & $\mathrm{E} 4.41, \mathrm{E} 4.4122, \mathrm{H} 2.6$ & & & \\
\hline 834. & Pulicaria dysenterica (L.) Bernh. & $\mathrm{H}$ & Euri-Medit. & L-637 & $\bullet$ & & & E3.4 & & & \\
\hline
\end{tabular}


Annex. 1 - cont.

\begin{tabular}{|c|c|c|c|c|c|c|c|c|c|c|c|}
\hline & FAMILY / Taxon & Life & Flor. element & Herb. & R.84 & R.90 & XK & EUNIS code & EU & END & Note \\
\hline 835. & Scorzonera austriaca Willd. & $\mathrm{H}$ & Euro-Siber. & L-638 & - & & & G1.7, E5.43, E1.22 & & & \\
\hline 836. & Scorzoneroides autumnalis (L.) Moench & $\mathrm{H}$ & Euro-Siber. & N/A & & - & & E5.51, E5.5721 & & & \\
\hline 837. & Senecio nemorensis subsp. bulgaricus (Velen.) Greuter & $\mathrm{H}$ & Balkan & L-468 & • & • & & $\begin{array}{l}\text { F2.22, G1.6933, G4.6, } \\
\text { G3.1 }\end{array}$ & & & \\
\hline 838. & Senecio squalidus subsp. rupestris (Waldst. \& Kit.) Greuter & $\mathrm{H}$ & Orof.SE.Eur. & L-469 & • & - & & F2.2, E5.43, G4.6 & & & \\
\hline 839. & Senecio vulgaris L. & $\mathrm{T}$ & Paleotemp. & L-639 & • & & & E5.43 & & & \\
\hline 840. & Serratula tinctoria $\mathrm{L}$. & $\mathrm{T}$ & Euro-Siber. & L-640 & • & & & E5.43, E5.2 & & & \\
\hline 841. & Tanacetum corymbosum (L.) Sch. Bip. & $\mathrm{H}$ & Euri-Medit. & L-448 & • & & & E1.7, E1.71, E1.73 & & & \\
\hline 842. & Tanacetum larvatum (Pant.) Hayek & c & Balkan & L-346 & & & & E3.4, E5.43 & & $\operatorname{End}(\mathrm{B})$ & $\boldsymbol{\nabla}$ \\
\hline 843. & Tanacetum macrophyllum (Waldst. \& Kit.) Sch. Bip. & $\mathrm{H}$ & Pontic. & L-449 & - & & & G1.6933, G4.6, E5.2 & & & \\
\hline 844. & Tanacetum parthenium (L.) Sch. Bip. & $\mathrm{H}$ & Euri-Medit. & L-450 & - & & & E5.4, G4.6, E5.2 & & & \\
\hline 845. & $\begin{array}{l}\text { Taraxacum alpinum (Hoppe.) Heg. \& Heer.- Handel-Mazz. } \\
\text { [Taraxacum sect. Erythrosperma (H. Lindb.) Dahlst.] }\end{array}$ & $\mathrm{H}$ & Circumbor. & L-476 & & & & E4.392 & & & \\
\hline 846. & $\begin{array}{l}\text { Taraxacum laevigatum (Willd.) DC. } \\
\text { [Taraxacum sect. Erythrosperma (H. Lindb.) Dahlst.] }\end{array}$ & $\mathrm{H}$ & Paleotemp. & N/A & & • & & E1.22, E1.73 & & & \\
\hline 847. & $\begin{array}{l}\text { Taraxacum officinale F. H. Wigg. (group) } \\
\text { [Taraxacum sect. Taraxacum F. H. Wigg.] }\end{array}$ & $\mathrm{H}$ & Circumbor. & L-641 & - & - & & E1.2, E3.3 & & & \\
\hline 848. & Taraxacum palustre (Lyons) Symons & $\mathrm{H}$ & Euro-Asiat. & N/A & • & & & G4.6, G1.6933 & & & \\
\hline 849. & Telekia speciosa (Schreb.) Baumg. & $\mathrm{H}$ & Euro-Asiat. & L-453 & - & & & E5.4, E5.43, E5.2 & & & \\
\hline 850. & Tephroseris papposa subsp. wagneri (Degen) B. Nord. & $\mathrm{H}$ & Balkan & L-461 & - & - & VU & E4.3411, E4.39, E4.392 & & $\operatorname{End}(\mathrm{B})$ & \\
\hline 851. & Tragopogon pratensis subsp. orientalis (L.) Čelak. & $\mathrm{H}$ & Euro-Siber. & N/A & - & & & E1.7 & & & \\
\hline 852. & $\begin{array}{l}\text { Willemetia stipitata subsp. albanica (Kümmerle \& Jáv.) } \\
\text { Kirschnerová }\end{array}$ & $\mathrm{H}$ & Balkan & L-462 & & & & D2.282 & & End(B) & \\
\hline 853. & Xeranthemum annuum L. & $\mathrm{T}$ & Euro-Asiat. & L-642 & - & & & $\mathrm{E} 1.2, \mathrm{E} 1.73, \mathrm{E} 4.43$ & & & \\
\hline
\end{tabular}

Table commentary:

FAMILY / Taxon - Plant family, followed by plant taxa (species and/or subspecies), Life - life form, Flor. element - Floristic element of the taxa, Herb. - Unique Herbarium Code of the Taxa (when indicated N/A, there is no Herbarium reference on the particular taxa, only literature data are used as reference), R.1984Rexhepi, 1984 (see References), R.1990 - Rajevski, 1990 (see References), XK - Status of plant taxa based on the Kosovarian Red List of plant taxa, EUNIS code - Habitat type according to EUNIS, EU - Red List status of European vascular plants, END - Status concerning Endemism, when so - 'End(B) is added, meaning Endemic of Balkans, Note - Additional notes concerning the plant taxa, $\boldsymbol{\nabla}$ - new locality in Kosovo, $\mathbf{-} \mathbf{-}-$ First record for Kosovo, CONS. + - Plant conservation and protection measures proposed. 


\section{Acknowledgement}

We are grateful to prof. V. Vladimirov (IBER - Bulgarian Academy of Sciences) for provided help in the final taxonomic identification of some critical Hieracium and Pilosella samples. We thank also prof. F. Rexhepi (University of Prishtina) for his helpful remarks during the initial stages of our survey. The article benefited greatly from comments of two anonymous reviewers

\section{References}

Ade A. (1954): Ein Ausflug in die Rudoka Gebirge bei Tetovo (Mazedonien) vom 3.6.1931 bis 5.6.1931. - Fragmenta Balcan. Mus. Macedon. Sci. Natur. 1(4): 31-35.

Amiđžić L., Bartula M. \& Jarić S. (2012): Syntaxonomy overview of vegetation of Šar Planina in the Balkan Peninsula. - Vegetos 25(2): 348-360.

Anonymous (2013): Spatial Plan of Sharri National Park. - MESP - Agency for Protection of Nature of Kosovo. Prishtina.

Anonymous (2014a): Red List of Vascular Flora of the Republic of Kosovo. - MESP - Agency for Protection of Nature of Kosovo. Prishtina.

Anonymous (2014b): Management Plan with the 10 year strategy for Sharri National Park. MESP - Prishtina.

Anonymous (2015): Report on the state of nature 2015-2017. - MESP - Agency for Protection of Nature of Kosovo. Prishtina.

Barina Z., Somogyi G., Pifkó D., \& Rakaj M. (2018): Checklist of vascular plants of Albania. Phytotaxa 378(1), 1. doi: 10.11646/phytotaxa.378.1.1

Berisha N., Millaku F., Gashi B. \& Matevski V. (2019): Ramondo-Ostryetum carpinifoliae - a new association from the hop-hornbeam forests of the Sharri Mountains, Kosovo. Hacquetia 18(2): 323-336. doi.org/10.2478/hacq-2019-0002

Bilz M., Kell S. P., Maxted N. \& Lansdown R. V. (2011): European Red List of Vascular Plants. - Luxembourg: Publications Office of the European Union.

Bridson D. \& Forman L. (eds) (1998): The Herbarium Handbook, 3rd edition. - Royal Botanic Gardens, Kew, Great Britain.

Calabrese V., Carranza M., Evangelista A., Marchetti M., Stinca A., \& Stanisci A. (2018): LongTerm Changes in the Composition, Ecology, and Structure of Pinus mugo Scrubs in the Apennines (Italy). - Diversity 10(3), 70. doi: 10.3390/d10030070

Čarni A. \& Matevski V. (2010): Vegetation along mountain streams in the Southern part of the Republic of Macedonia. - Braun-Blanquetia 46: 157-170.

Çavolli R. (1997): Regional geography of Kosovo. - ETMMK, Prishtina. [in Albanian]

Council of Europe (1979): Convention on the Conservation of European Wildlife and Natural Heritage. - Bern, Switzerland. Available at:

http://conventions.coe.int/Treaty/EN/Treaties/Html/104.htm

Diklić N. \& Stevanović V. (2012): Dianthus. - In: Stevanović V. (ed). The Flora of Serbia 2. SANU. Belgrade. pp. 547. [in Serbian]

Dimopoulos P., Raus T., Bergmeier E., Constantinidis T., latrou G., Kokkini S., Strid A. \& Tzanoudakis D. (2016): Vascular plants of Greece: An annotated checklist. Supplement. Willdenowia 46(3): 301-347. doi: 10.3372/wi.46.46303

Dörfler I. (1894-1914): Jahres-Katalog des Wiener botanischen Tauschvereins - Wien. 
EUNIS (2007): EUNIS Habitat Classification. Available at:

http://www.eea.europa.eu/themes/biodiversity/eunis/eunis-habitat-classification.

Euro+Med (2006+) [continuously updated]: Euro+Med PlantBase - the information resource for Euro-Mediterranean plant diversity. - Published at http://ww2.bgbm.org/EuroPlusMed/ [accessed 17 Aug 2019].

Gajić M. (1980): An overview of the flora species of SR Serbia with their geographical indications. - J. Fac. Forestry (Belgrade) 54: 111-141. [in Serbian]

Gentry A. H. (1978): Floristic knowledge and needs in Pacific Tropical America. - Brittonia 30(2): 134-153. doi: $10.2307 / 2806640$

Grisebach A. (1843): Spicelegium florae rumelicae et bithynicae exhibens synopsin plantarum quas aest. - Brunsvigae.

Horvat I. (1960): Macedonias mountain vegetation in the light of contemporary research. Special Edition of the Natural History Museum of Macedonia. Skopje. [in Serbo-Croatian] Horvat L., Glavač V. \& Ellenberg H. (1974): Vegetation Südosteuropas. - Gustav Fischer Verl. - Stuttgart.

Ivanović R., Valjarević A., Vukoćić D. \& Radovanović D. (2016): Climatic regions of Kosovo. Univ. Thought 6(1): 49-54. doi:10.5937/univtho6-10409.

Jäger E., Johannes R. C. M., Müller F., Welk E. \& Wesche K. (2013): Rothmaler - Exkursionsflora von Deutschland: Gefäßpflanzen: Atlasband (12. Aufl.). - Heidelberg: Springer Verlag Berlin. [in German] doi: doi.10.1007/978-3-8274-2723-6

Josifović M. (ed.) (1970-1977): Flora of SR of Serbia - volumes: 1-9. - Serbian Academy of Sciences and Arts. Belgrade [in Serbian]

Kendall P. \& Snelson B. (2009): The role of floristic survey data and quantitative analysis in identification and description of ecological communities under threatened species legislation: A case study from north-eastern New South Wales. - Ecol. Manag. Restor. 10(1): S16-S26. doi: 10.1111/j.1442-8903.2009.00454.x

Krivošej Z. (1997): Vascular flora of the Mt. Oshlak, Sharri Mts. - PhD Thesis (msc.). Faculty of Biology. University of Belgrade. [in Serbian]

Martin J. L. (2009): Are the IUCN standart home-range thresholds for species a good indicator to prioritise conservation urgency in small islands? A case study in the Canary islands (Spain). - J. Nat. Conserv. 17(2): 87-98. https://doi.org/10.1016/j.jnc.2008.10.001

Matevski V. \& Kostadinovski M. (1999): Contribution to the flora of the Republic of Macedonia - III. - Bull. FNS - Ss Cyril \& Methodius University in Skopje. 52: 102-108. [in Macedonian].

Matevski V. (ed) (2010): The Flora of the Republic of Macedonia. 2nd volume. - Macedonian Academy of Sciences and Arts. Skopje. [in Macedonan]

Matevski V., Čarni A., Ćušterevska R., Kostadinovski M., Mucina L. (2018): Syntaxonomy and biogeography of dry grasslands on calcareous substrates in the central and southern Balkans. - Appl. Veg. Sci. 21(3): 488-513. doi: 10.1111/avsc.12374

Micevski K. \& Matevski V. (1987): The territorial division of endemics in the Republic of Macedonia and the problem of their endangerment. - Academy of Sciences and Arts of $\mathrm{BiH}$ - Special Edition. pp. 199-205. [in Serbo-Croatian]

Micevski, K. (ed.) (1985-2005): Flora of the Republic of Macedonia. Volumes: 1 - 5. Macedonian Academy of Sciences and Arts. Skopje [in Macedonian]

Micevski K. (1995): Flora of the Republic of Macedonia 1(3). - Macedonian Academy of Sciences and Arts. Skopje. [in Macedonian]

Micevski K. (2001): Flora of the Republic of Macedonia 1(5). - Macedonian Academy of Sciences and Arts. Skopje. [in Macedonian] 
Millaku F., Heiselmayer P., Rexhepi F., Krasniqi E., Eichberger Ch. \& Haziri A. (2008): Endemic, stenoendemic and relic plants in serpentines of Kosovo. - Sauteria 16: 149-161.

Millaku F., Krasniqi E. \& Rexhepi F. (2011): The Association Stipeto-Convolvuletum compacti ass. nova in Kosovo. - Hacquetia 10(20): 137-147. doi.org/10.2478/v10028-011-0008-4

Millaku F., Rexhepi F., Krasniqi E., Abdullai K. \& Mala Xh. (2012): Mediterranean floristic element and its influence in the alliance Ostryo-Carpinion orientalis HT. 1954 in Kosovo. - Bocconea 24: 295-300.

Millaku F. (ed), Rexhepi F., Krasniqi E., Pajazitaj Q., Mala Xh., Berisha N. (2013): The Red Book of vascular flora of the Republic of Kosovo. - MESP. Prishtina.

Millaku F., Krasniqi E., Berisha N. \& Rexhepi F. (2017): Conservation assessment of the endemic plants from Kosovo. - Hacquetia 16(1): 35-47. doi:10.1515/hacq-2016-0024

Orsenigo S., Montagnani C., Fenu G., Gargano D., Peruzzi L., Abeli T. \& Rossi G. (2018): Red Listing plants under full national responsibility: Extinction risk and threats in the vascular flora endemic to Italy. - Biol. Conserv. 224: 213-222. doi: 10.1016/j.biocon.2018.05.030

Paparisto K., Demiri M., Mitrushi I. \& Qosja Xh. (1988): Flora of Albania I. - Albanian Academy of Sciences and Arts. The Biological Research Center - Tirana. [in Albanian]

Petrova A. \& Vladimirov V. (2010): Balkan endemics in the Bulgarian flora. - Phytol Balc. 6(2): 293-311.

Pignatti S. (1982): Flora d'Italia vol.1-3. - Bologna: Edagricole. [in Italian]

Prodanović D., Krivošej Z., Stanojević M. \& Ćirić M. (2018): Supplement to the phytogeographical studies of the rare and internationally significant species in the flora of Serbia, Kosovo and Metohija. - Univ. Thought 8(1):10-16. doi:10.5937/univtho8-15361

Qosja Xh., Paparisto K., Demiri M., Vangjeli J. \& Balza E. (1992): Flora of Albania II. - Albanian Academy of Sciences and Arts. The Biological Research Center - Tirana. [in Albanian]

Qosja Xh., Paparisto K., Vangjeli J. \& Ruci B. (1996): Flora of Albania III. - Albanian Academy of Sciences and Arts. The Biological Research Center - Tirana. [in Albanian]

Rajevski L. (1990): Phytocenological characteristics of mountain pastures of the northern part of Sharri Mts. - Bull. Inst. Bot. Gard. Univ. Belgrade 9: 1-62. [in Serbian]

Rexhepi F. (1984): Flora of Luboten. Nature of Kosovo - EKMN 4: 27-54. [in Albanian]

Rexhepi F. (1994): Vegetation of Kosovo. - FNSM - University of Prishtina. Prishtina. [in Albanian]

Rexhepi F., Millaku F. \& Krasniqi E. (2005): Plant species and vegetation with endemic character in "Sharri" National Park, Kosovo. - In: 12th Inernational Botanical Congress. Vienna, Austria.

Rexhepi F., Millaku F., Krasniqi E. (2009): Some species of Mediterranean floristic element in Albanian Alps of Kosovo. - Bocconea 23(1): 294-300.

Rohlena J. (1937): Additamenta in floram Peninsulae Balcanicae. - Repert Spec. Nov. Regni. Veg. 42: 199-201. [in Latin]

Sarić M. \& Diklić N. (eds.) (1986): Flora of SR Serbia vol. 10 - Supplement. - Serbian Academy of Sciences and Arts. Belgrade. [in Serbian]

Shuka L., Xhulaj M., Kashta L. \& Casper J. (2007): The genus Pinguicula (Lentibulariaceae) in Albania - a critical review. - Wulfenia 14(1): 15-65.

Stevanović V. (ed). (2012): The flora of Serbia 2. - Serbian Academy of Sciences and Arts. Belgrade [in Serbian]

Stevanović V., Tan K. \& Petrova A. (2007): Mapping the endemic flora of the Balkans a progress report. - Bocconea 21(1): 131-137. 
Tomović G., Niketić M., Lakušić D., Ranđelović V. \& Stevanović V. (2014): Balkan endemic plants in Central Serbia and Kosovo regions: Distribution patterns, ecological characteristics, and centres of diversity. - Bot. J. Linn. Soc. 176(2): 173-202. doi:10.1111/boj.12197

Tutin T. G., Heywood V. H., Burges N. A., Moore D. M., Valentine D. H., Walters S. M. \& Webb D. A. (ed.) (1968-1980): Flora europaea. vol 2-5. - Cambridge.

Tutin T. G., Burges N. A., Chater A. O., Edmondson J. R., Heywood V. H., Moore D. M., Valentine D. H., Walters S. M. \& Webb D. A. (ed.) (1993): Flora europaea. vol 1 (2nd edition). - Cambridge.

Uzunov U. \& Gussev Ch. (2003): High mountain flora of Bulgaria - Statistics, ecological characteristics and phytogeography. - Bocconea 16(2): 763-770.

Vangjeli J., Ruci B., Mullaj A., Paparisto K. \& Qosja Xh. (2000): Flora of Albania IV. - Albanian Academy of Sciences and Arts. The Biological Research Center - Tirana. [in Albanian]

Veselaj Z., Mustafa B., Krasniqi F. \& Hoxha E. (2006): Species of international significance and their distribution in Kosovo. - Proceedings of the IVth Balkan Botanical Congress, Sofia, Bulgaria.

Vogt R., Lack H. W., \& Raus T. (2018): The herbarium of Ignaz Dörfler in Berlin. - Willdenowia 48(1): 57-92. doi:10.3372/wi.48.48105.

Received: March $1^{\text {st }} 2020$

Revised: May $8^{\text {th }} 2020$

Accepted: May $13^{\text {th }} 2020$ 